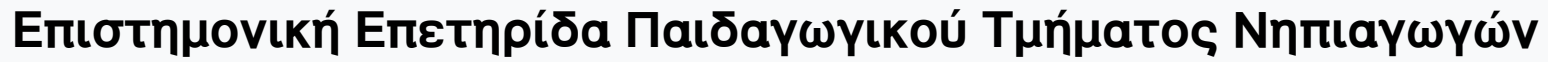

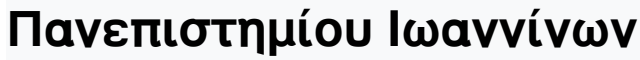

Tóp. 6 (2013)

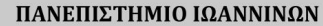

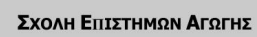

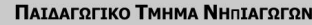

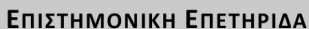

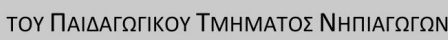

JOURNAL OF RESEARCH IN EDUCATION AND TRAINING

DEPARTMENT OF EARLY CHILDHOOD EDUCATION

TOMOE 6-VoL 6

2013

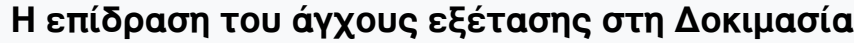

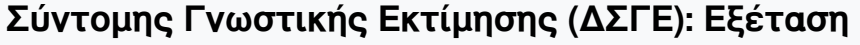

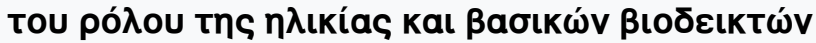

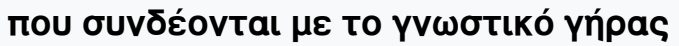

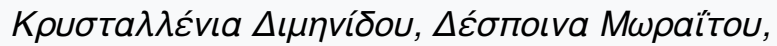

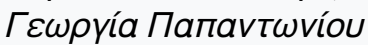

doi: $10.12681 /$ jret.761

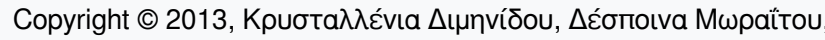

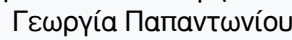

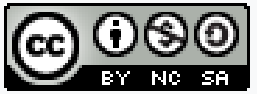

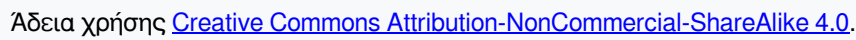

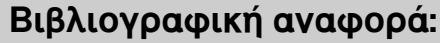

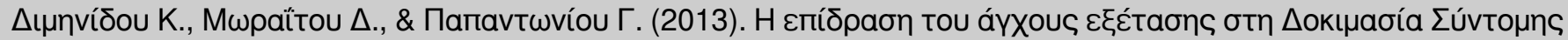

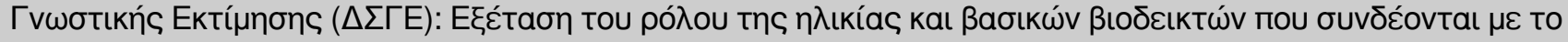

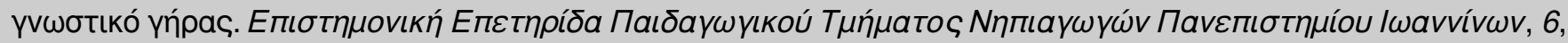
216-248. https://doi.org/10.12681/jret.761 


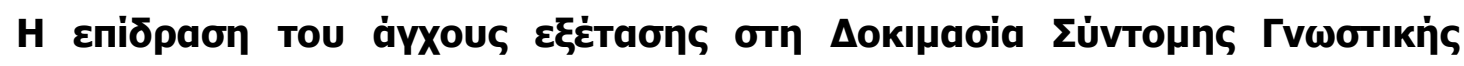

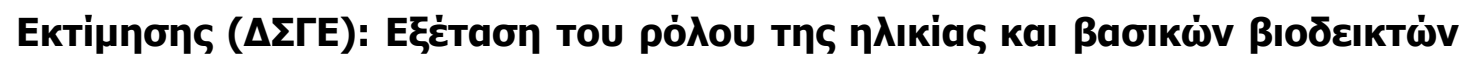

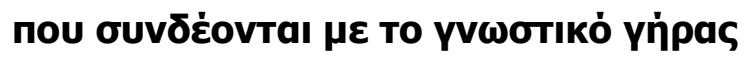

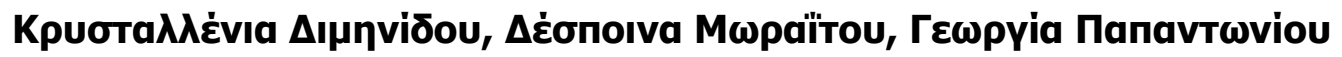

\section{ПЕРIАНЧН}

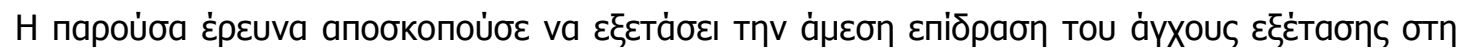

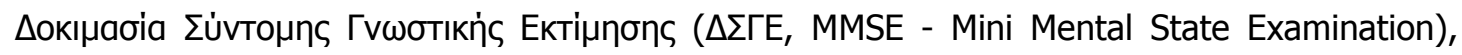

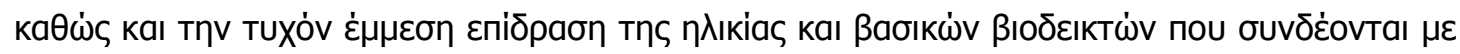

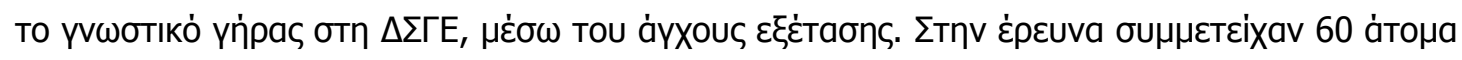

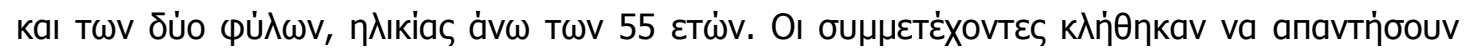

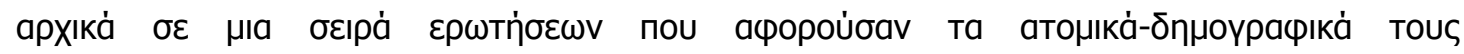

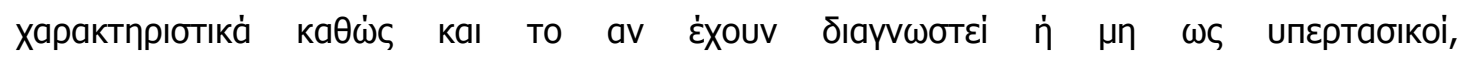

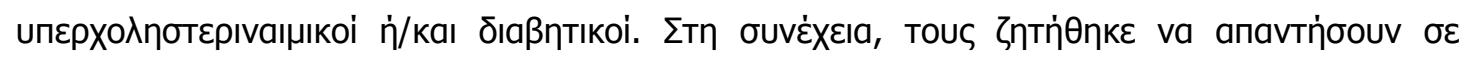

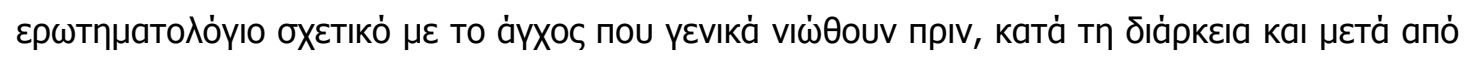

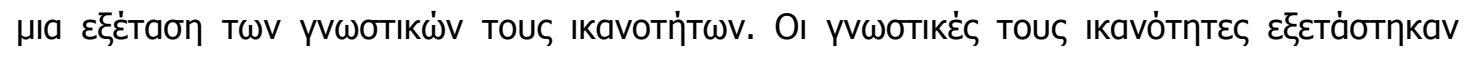

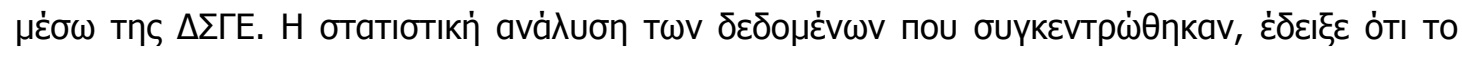

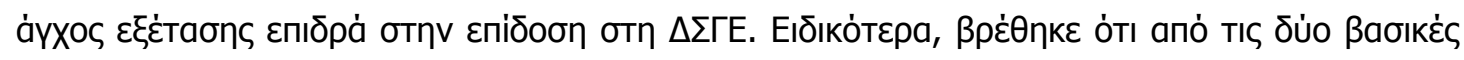

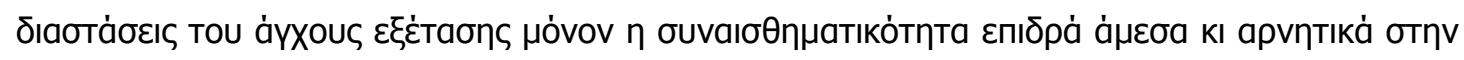

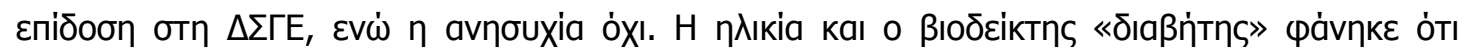

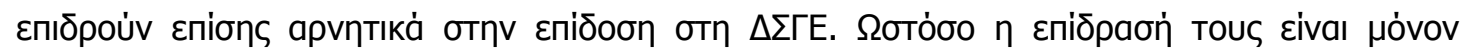

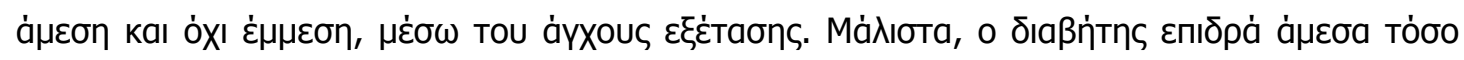

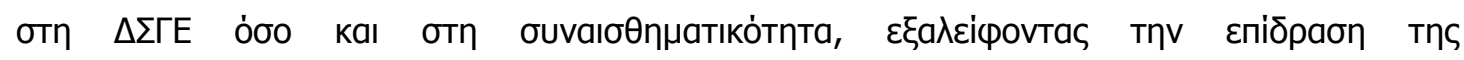

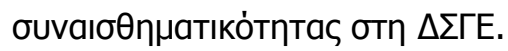

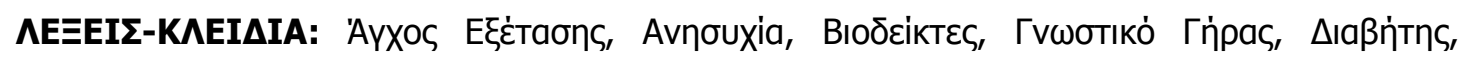

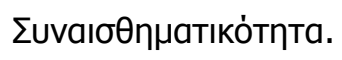




\title{
The effect of test anxiety on Mini Mental State Examination (MMSE): examining the role of age and basic biomarkers related to cognitive aging
}

\author{
Krystallenia Diminidou, Despina Moraitou, Georgia Papantoniou
}

\begin{abstract}
The present study aimed at investigating the direct effect of test anxiety on Mini Mental State Examination (MMSE) as well as the possible indirect effect of age and basic biomarkers related to cognitive aging, on Mini Mental State Examination (MMSE), through test anxiety. The sample was consisted of 60 persons aged 55 years and over. At first, participants were asked to answer a series of questions related to individual-demographic factors and whether they had a diagnosed hypertension, hypercholesterolemia, or/and diabetes. Then, they were asked to respond to an inventory tapping trait test anxiety generally felt before, during and after an examination of their cognitive abilities. They were also examined via the MMSE. The statistical analyses of the data gathered, showed that test anxiety affects MMSE performance. Specifically, it was found that from the two basic dimensions of test anxiety only emotionality has a direct negative effect on MMSE performance, while worry has not. Age and the biomarker "diabetes" were also found to influence negatively MMSE performance. However, their effects on MMSE performance were direct and not indirect, through test anxiety. Indeed, diabetes directly affects both MMSE performance and emotionality, eliminating in this way the effect of emotionality on MMSE performance.
\end{abstract}

KEY WORDS: Test anxiety, Worry, Biomarkers, Cognitive aging, Diabetes, Emotionality. 


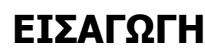

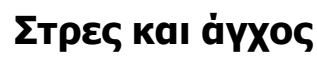

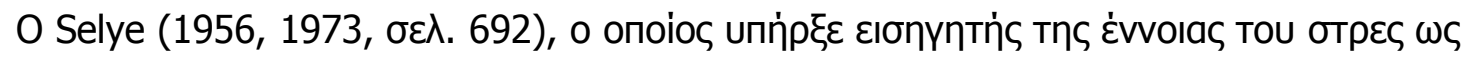

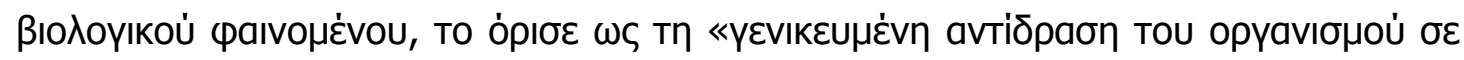

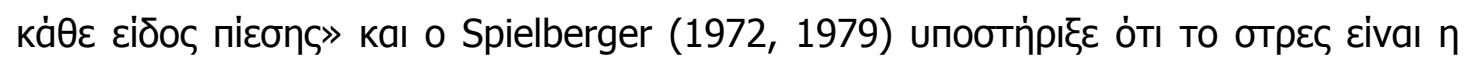

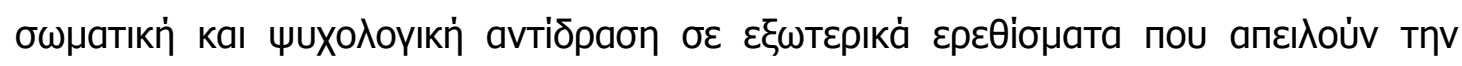

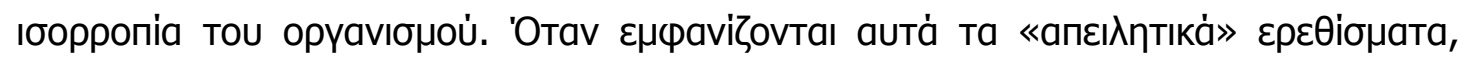

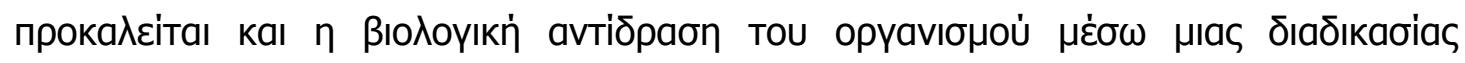

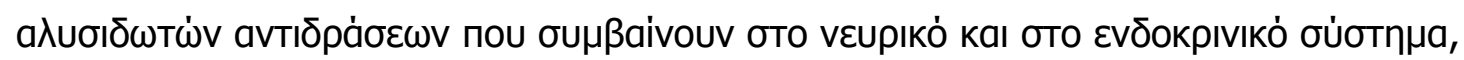

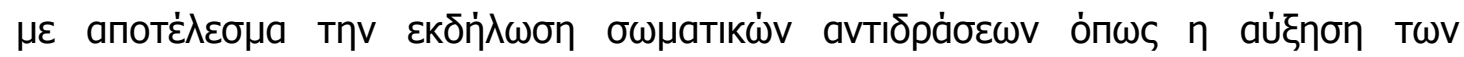

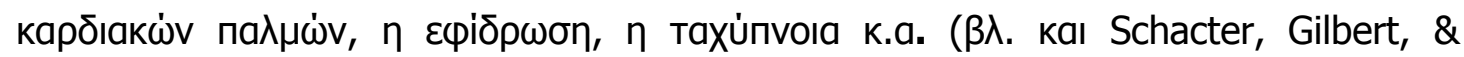
Wegner, 2012).

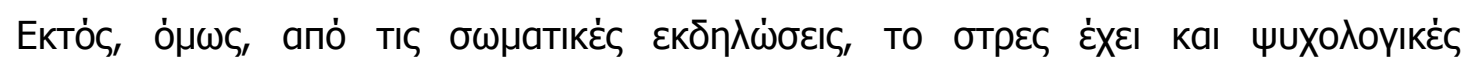

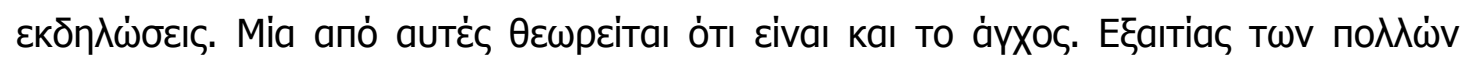

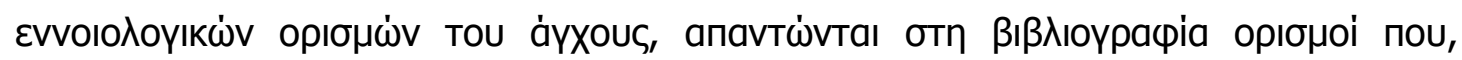

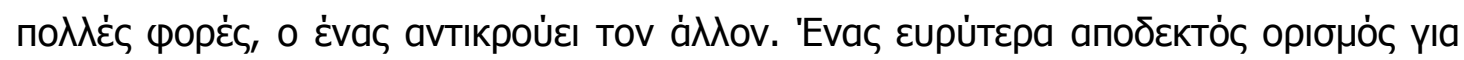

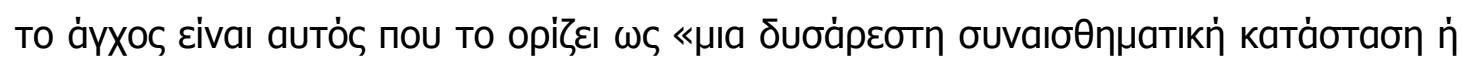

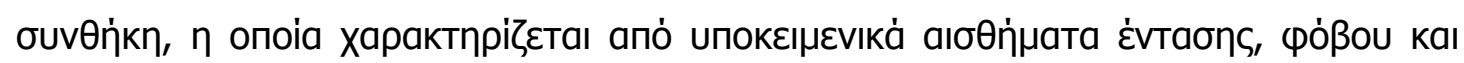

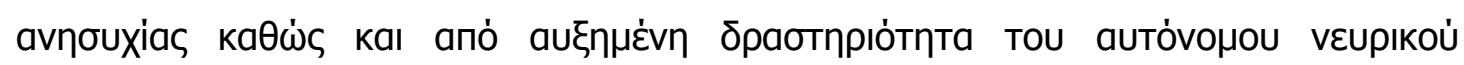

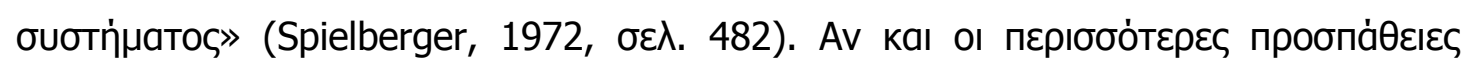

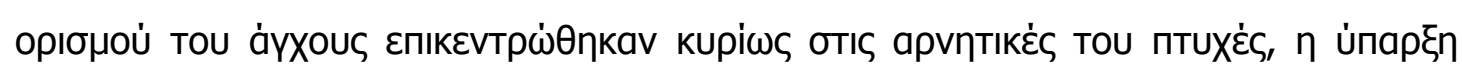

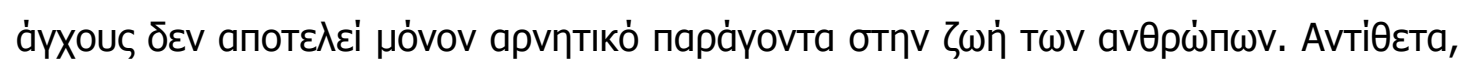

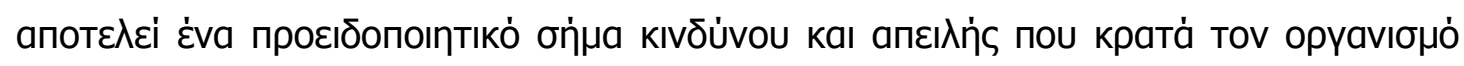

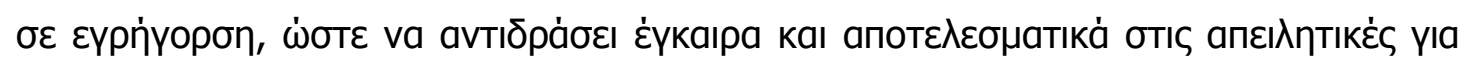

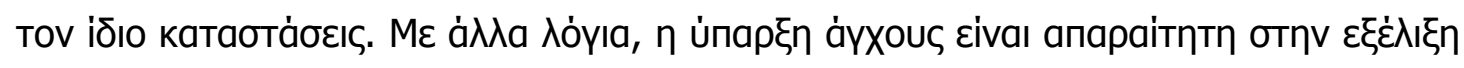

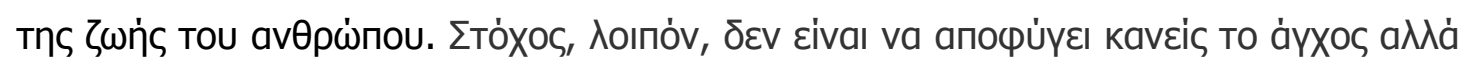

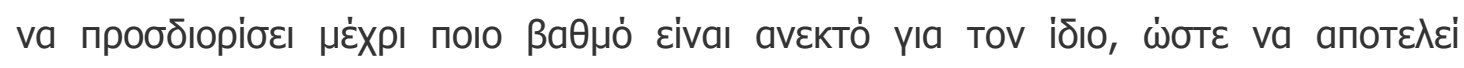

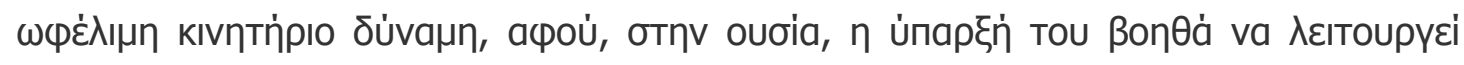

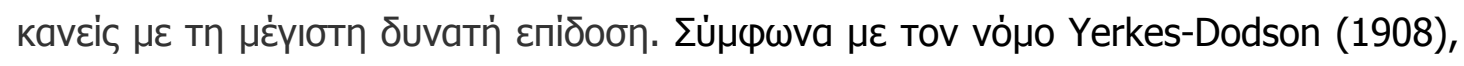

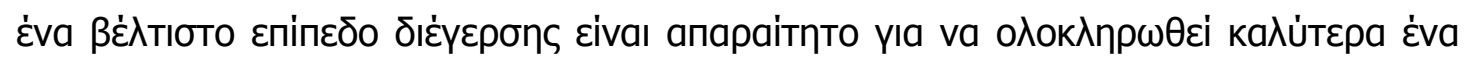

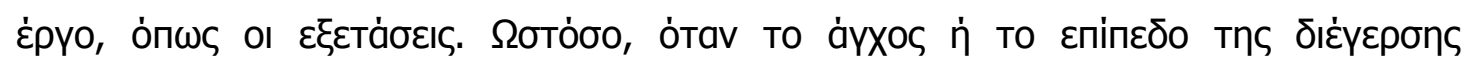

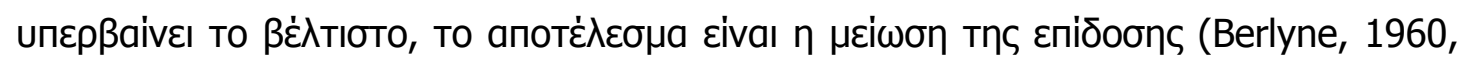
1963. Hebb, 1949, 1966. Yerkes \& Dodson, 1908). 


\section{'AYXos є乡ંंтaons}

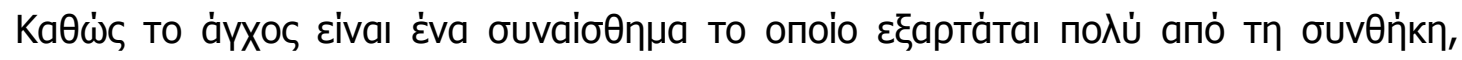

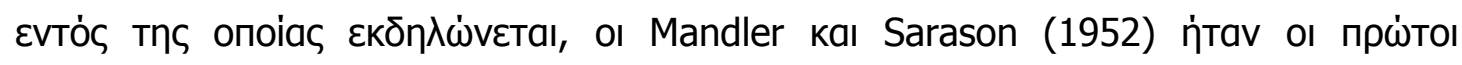

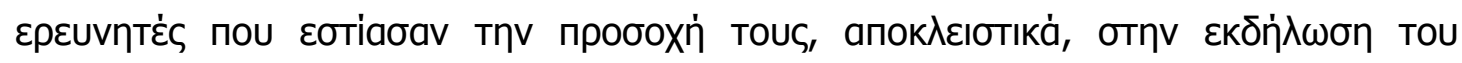

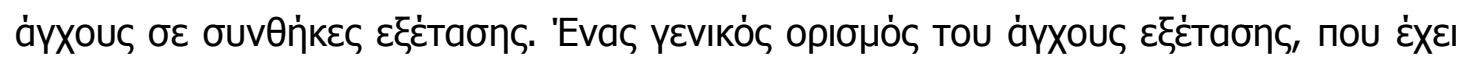

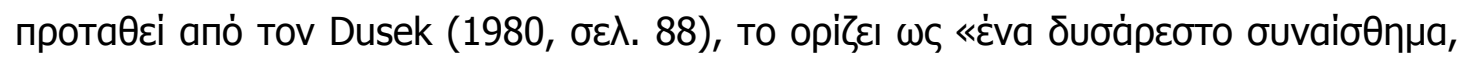

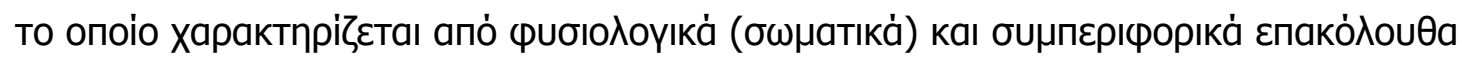

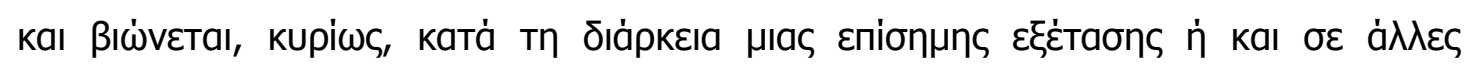

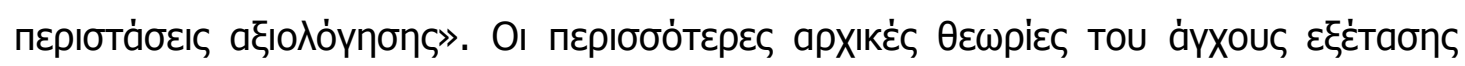

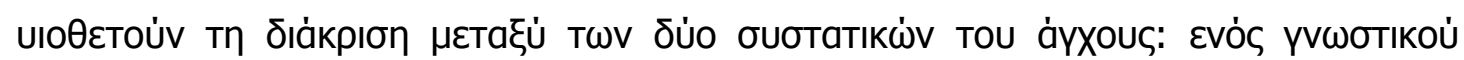

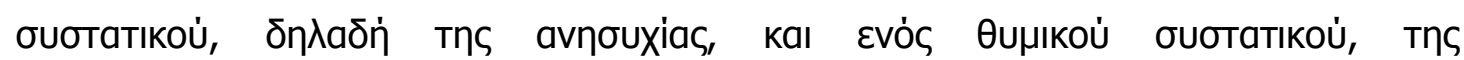
бuvaıбӨпнатıко̇тптаৎ (Liebert \& Morris, 1967. Schwarzer, 1984. Wigfield \& Eccles,

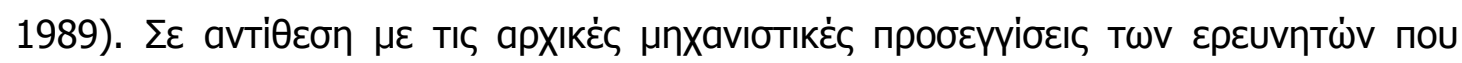

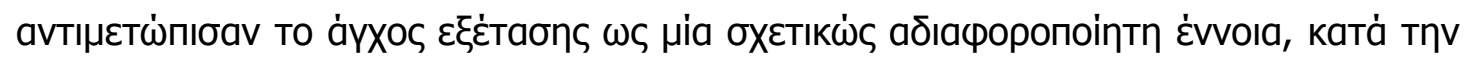

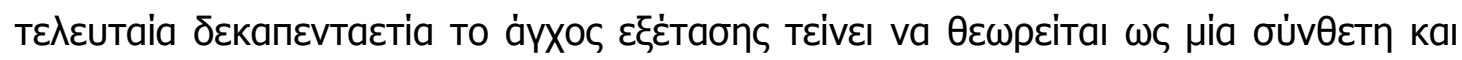

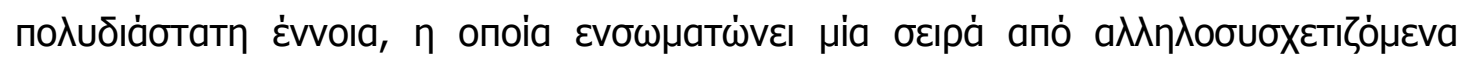

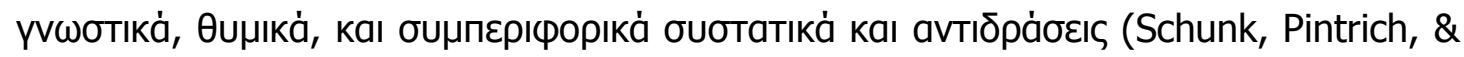
Meece, 2010. Zeidner, 1998).

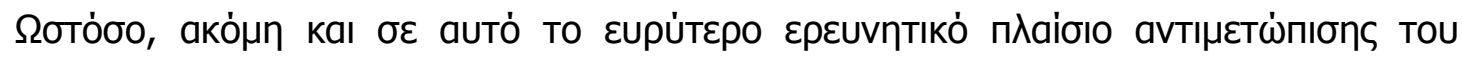

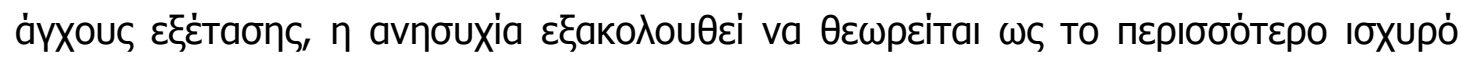

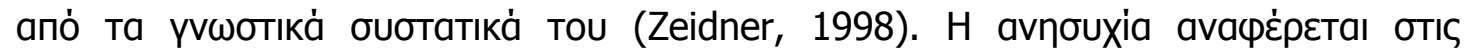

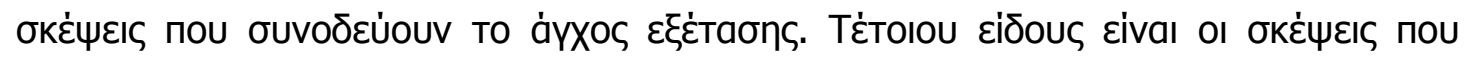

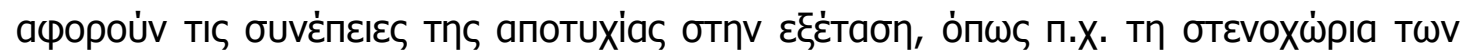

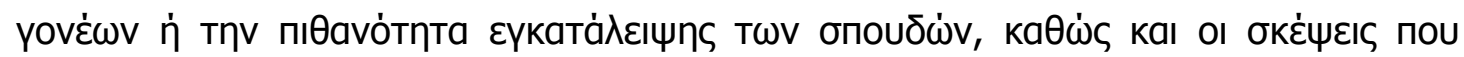

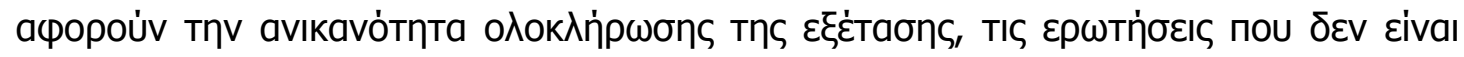

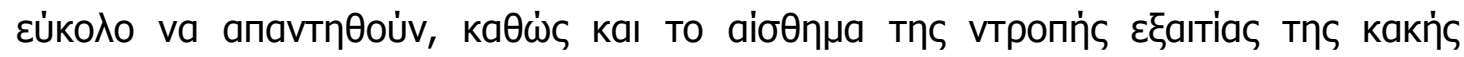

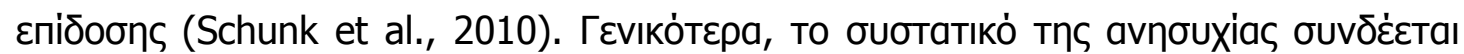

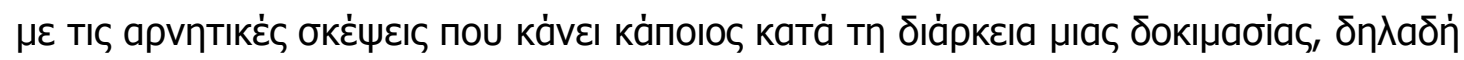

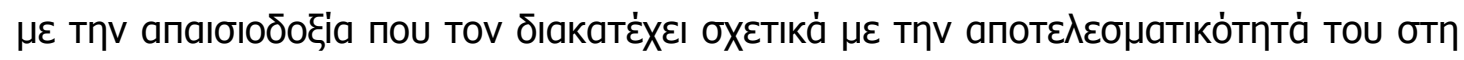

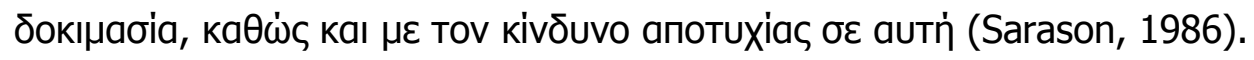

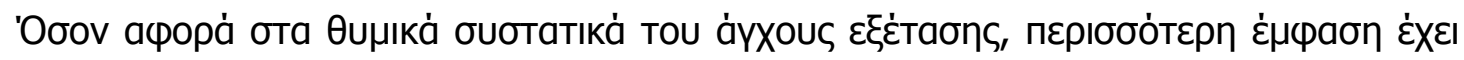

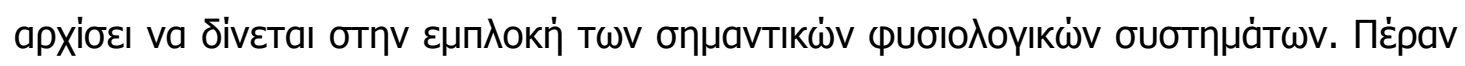

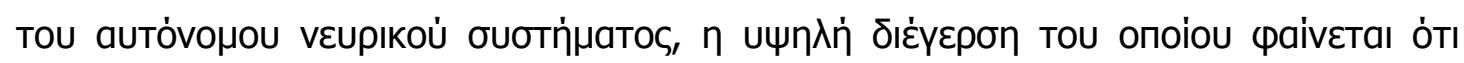




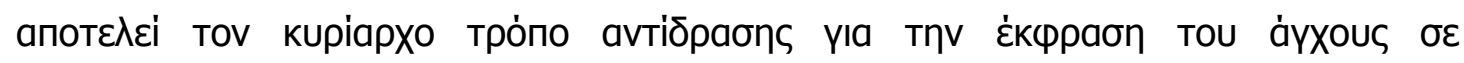

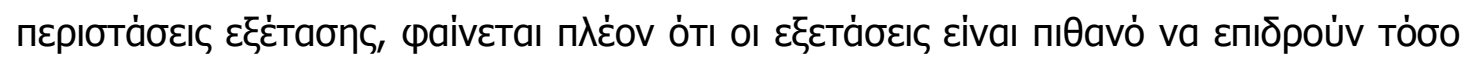

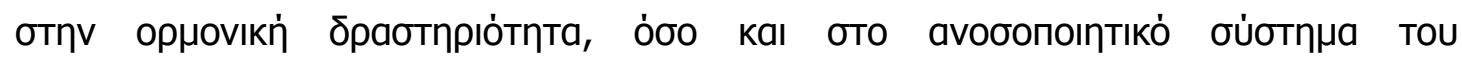

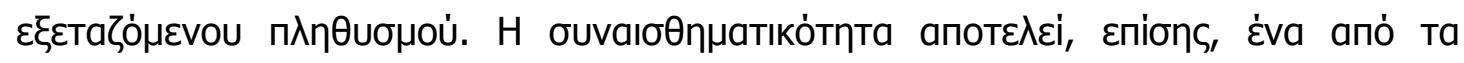

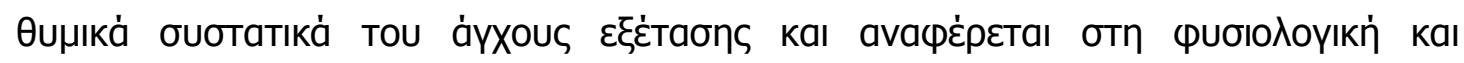

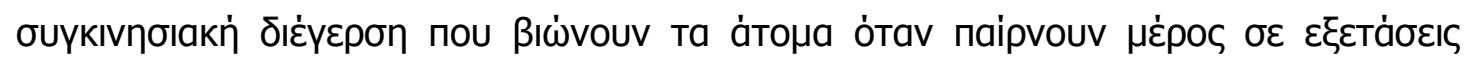

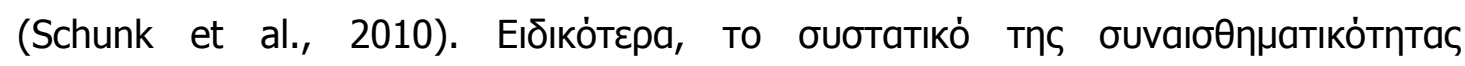

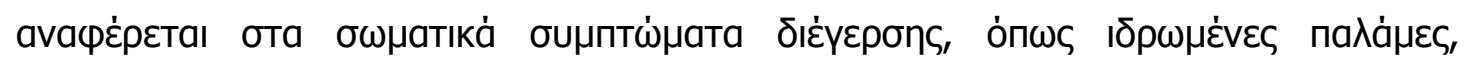

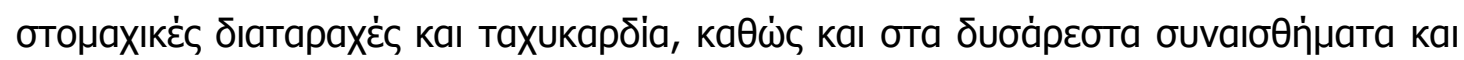

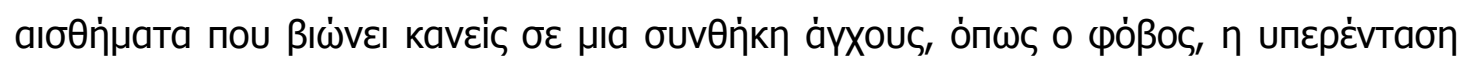

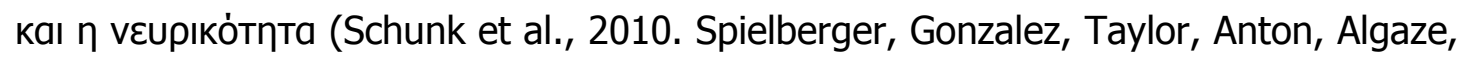

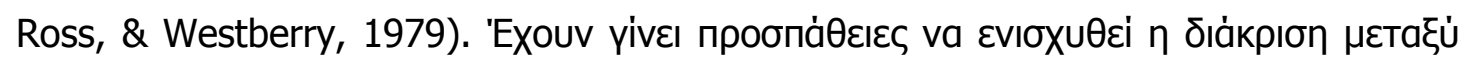

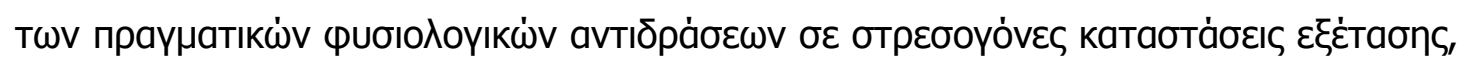

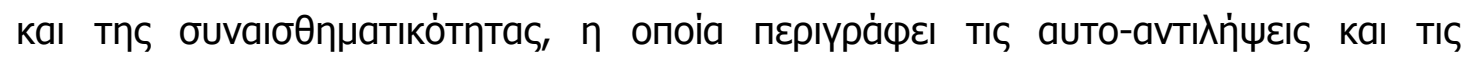

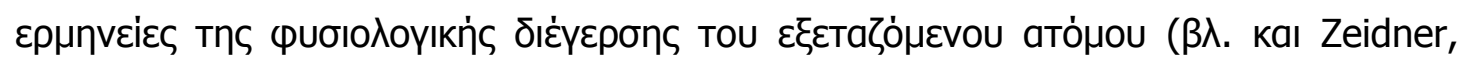

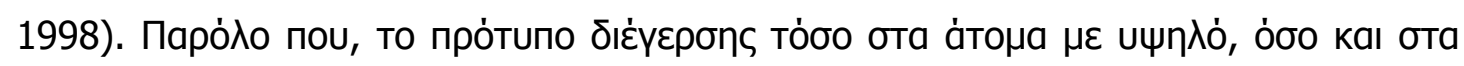

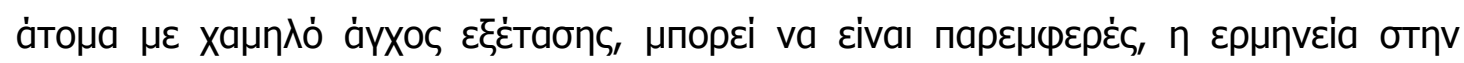

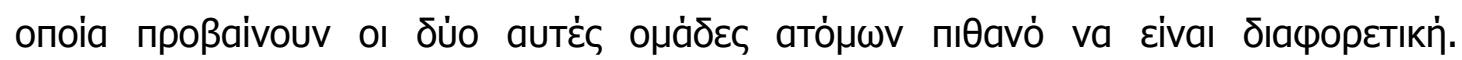

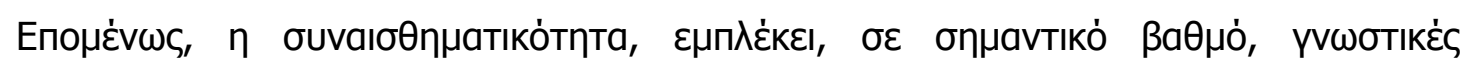

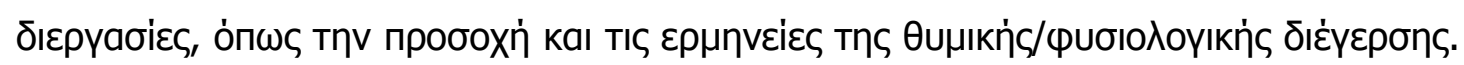

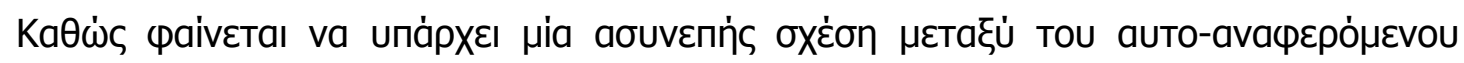

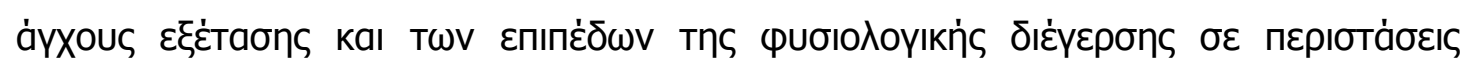

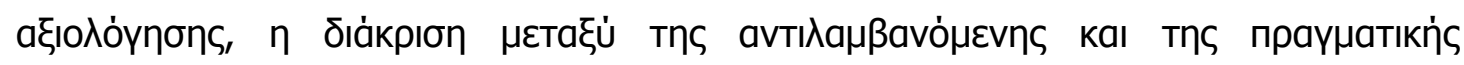

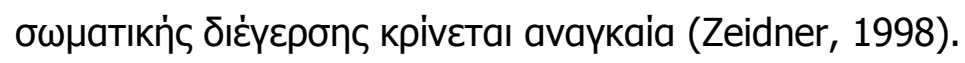

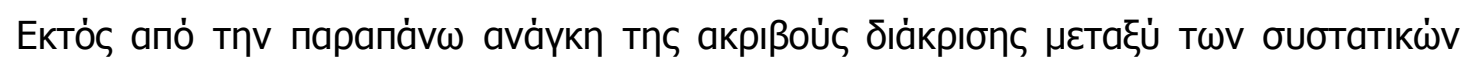

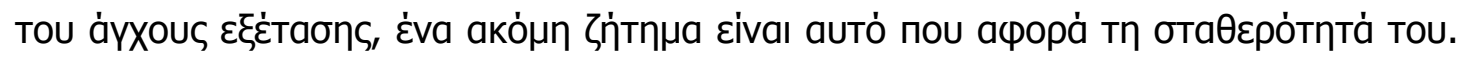

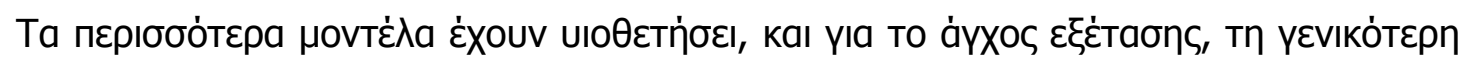

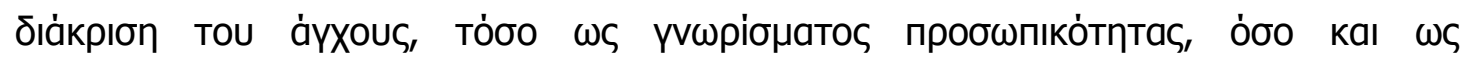

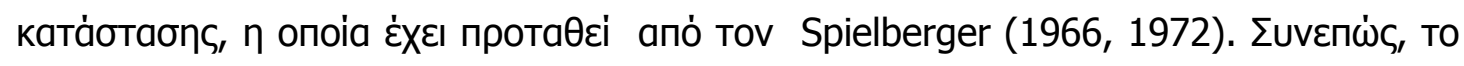

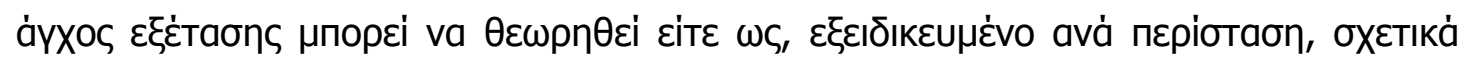

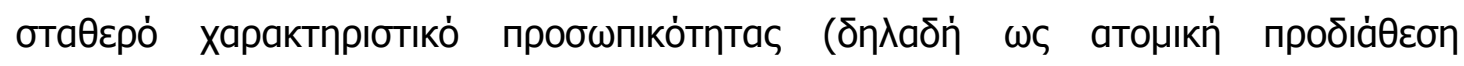

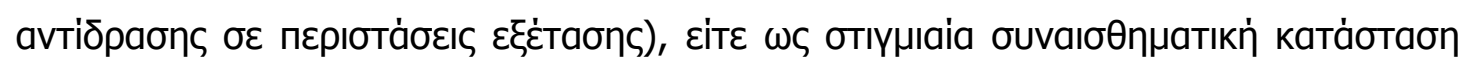

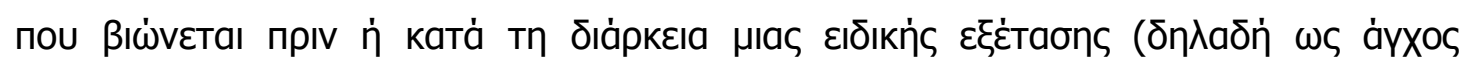

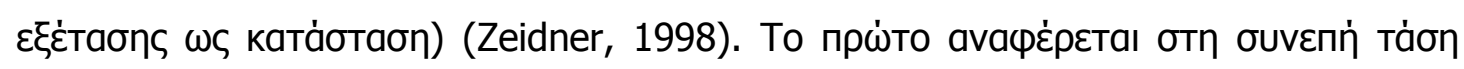




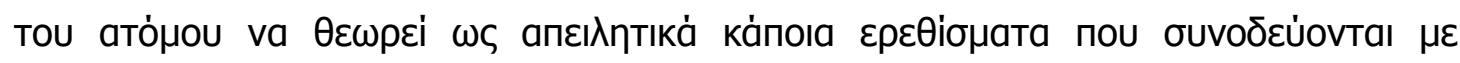

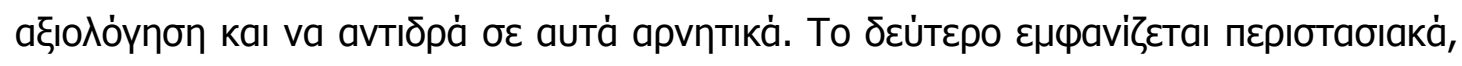

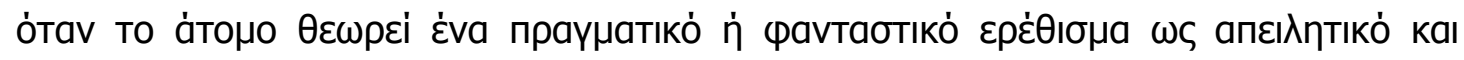

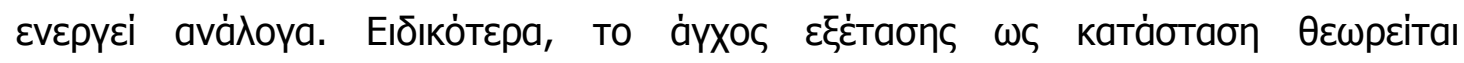

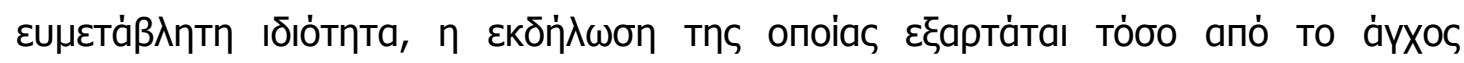

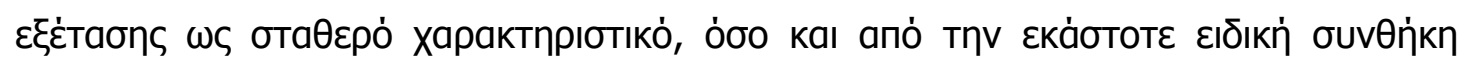

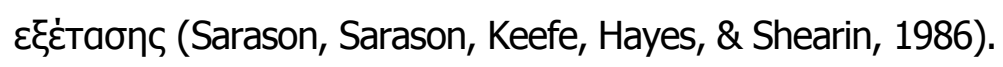

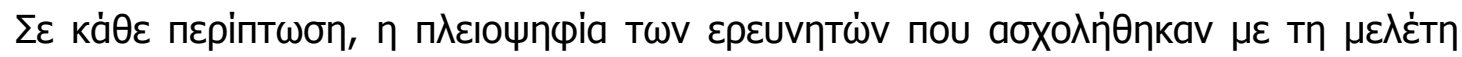

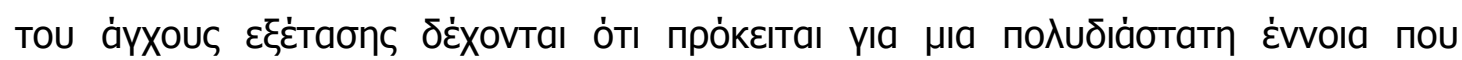

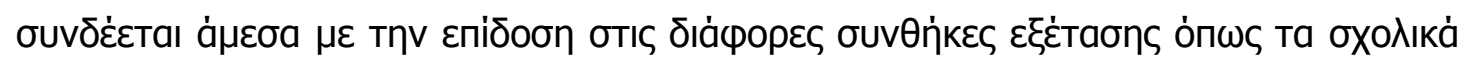

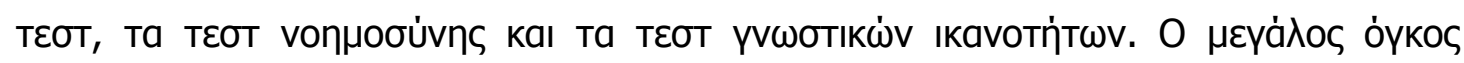

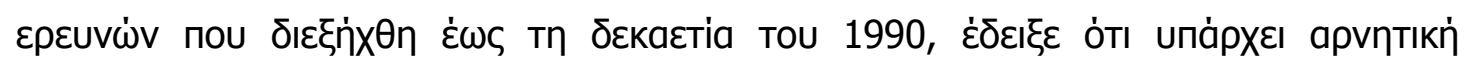

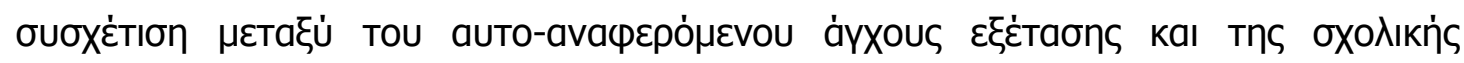

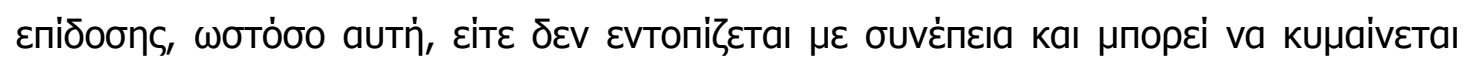

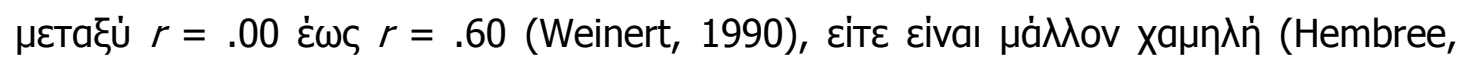

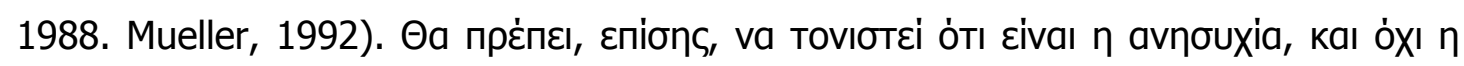

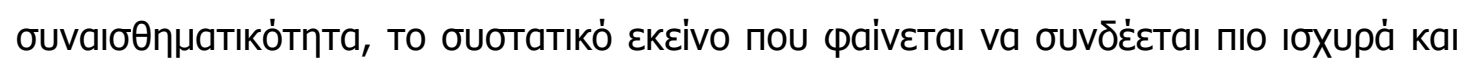

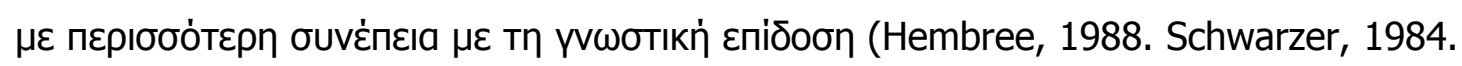

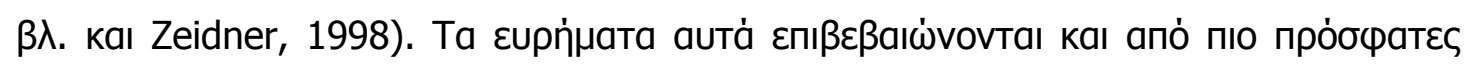

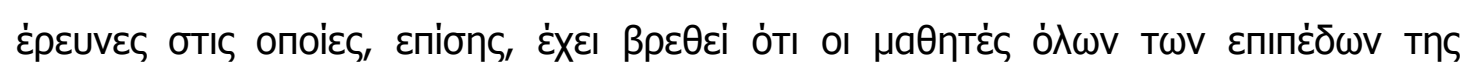

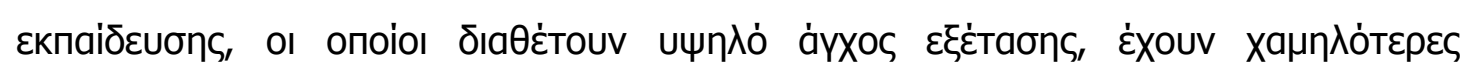

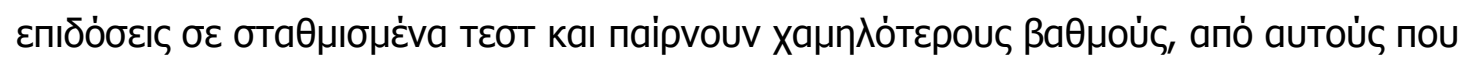

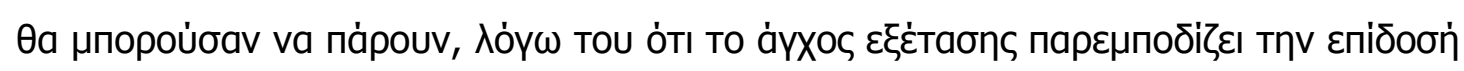

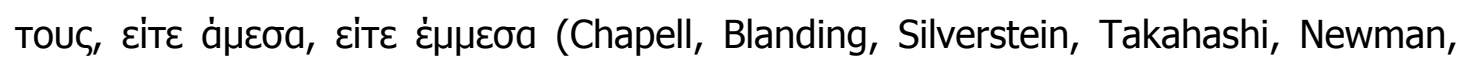
Gubi, \& McCann, 2005. Lowe, Lee, Witteborg, Prichard, Luhr, Cullinan, Mildren, Raad, Cornelius, \& Janik, 2008. Metallidou \& Vlachou, 2007. Manavtwviou, 2011. Sub

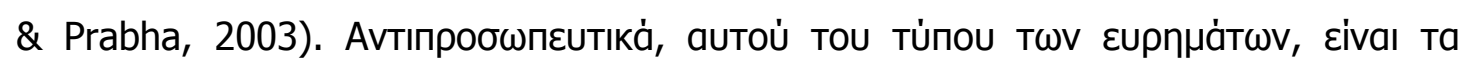

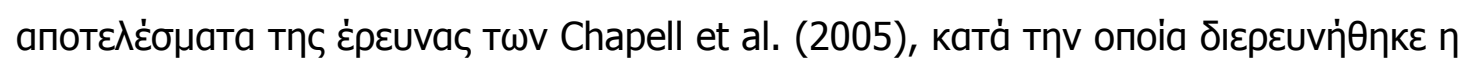

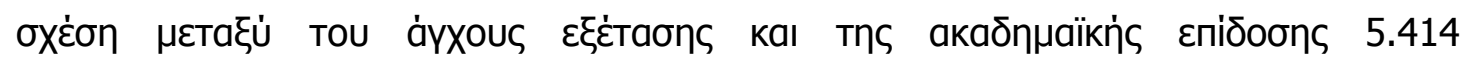

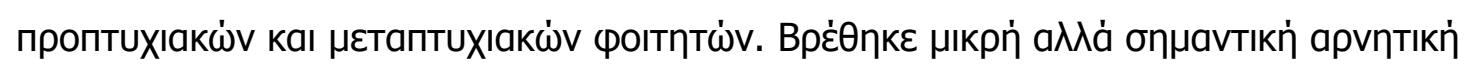

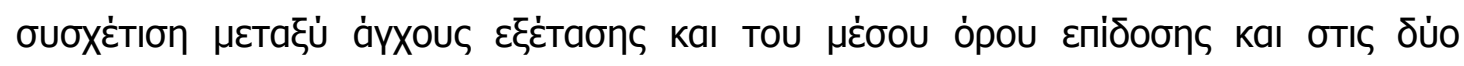

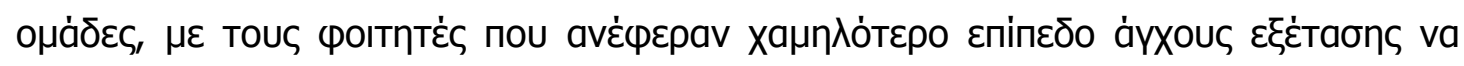

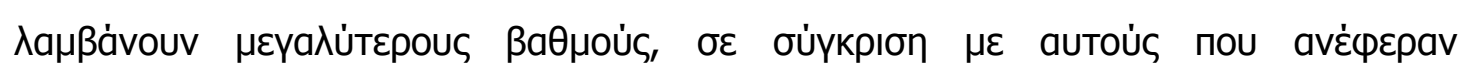

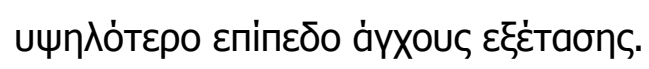




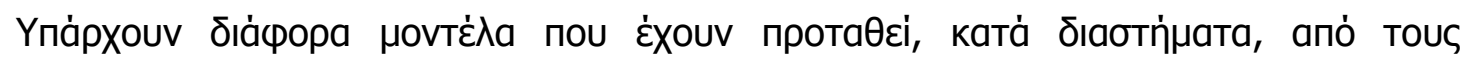

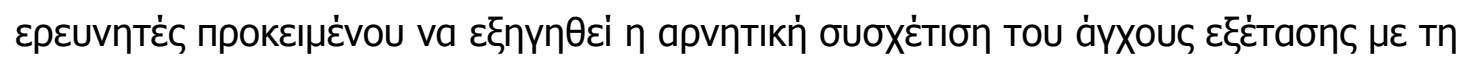

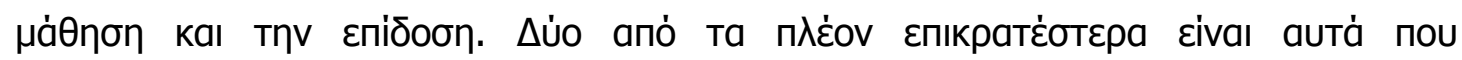

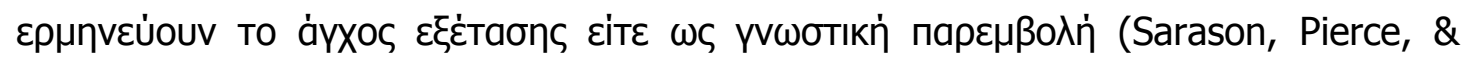

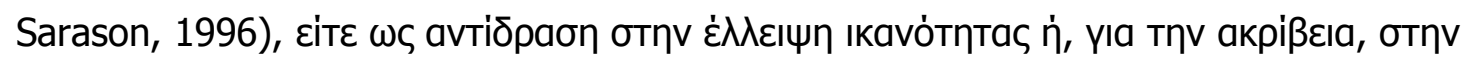

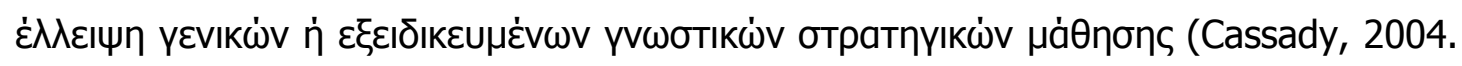

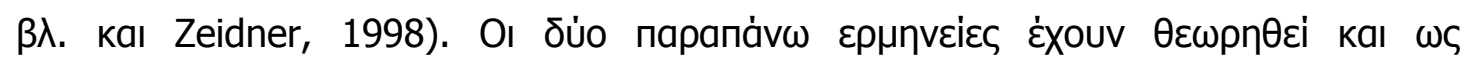

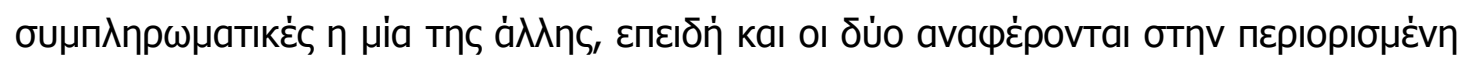

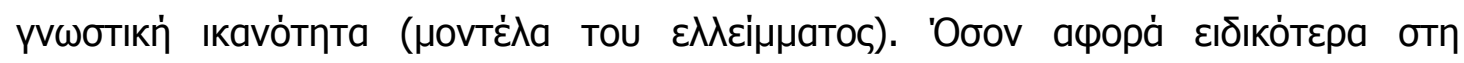

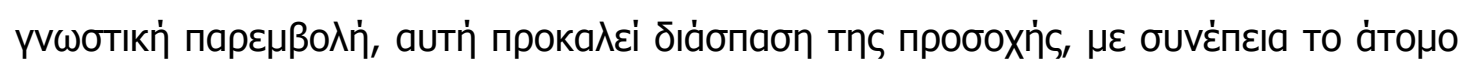

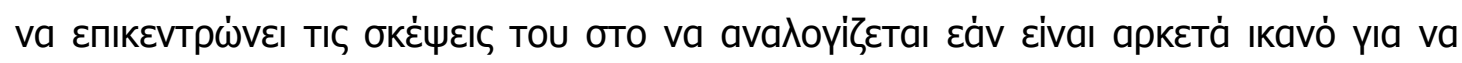

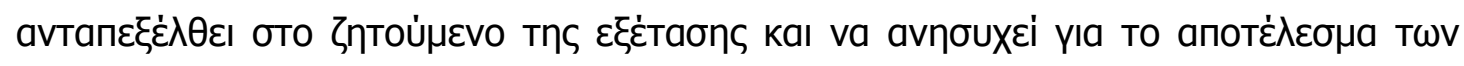

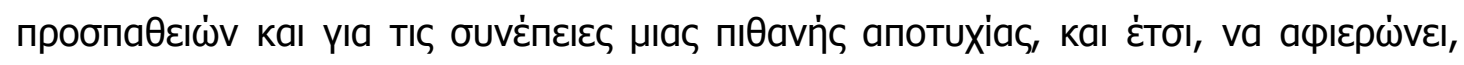

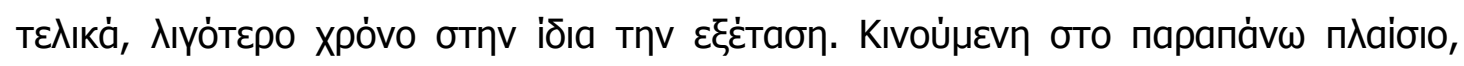

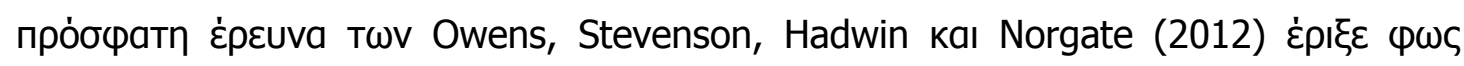

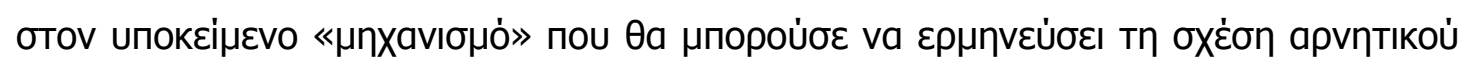

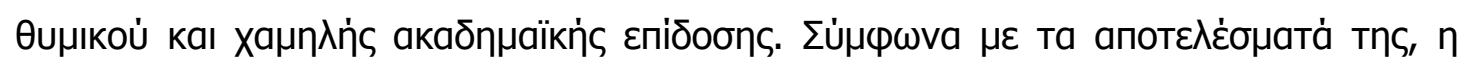

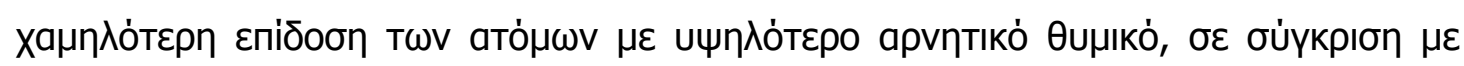

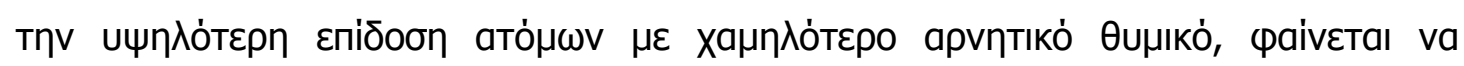

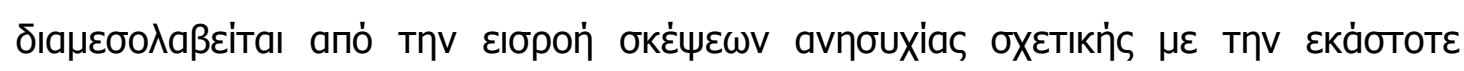

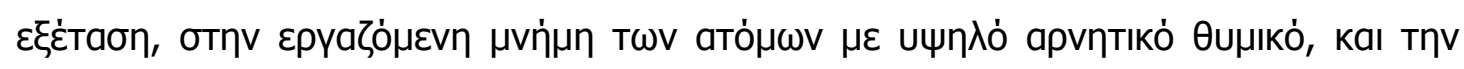

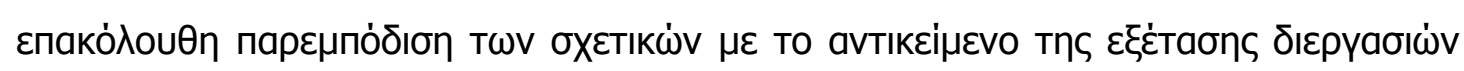

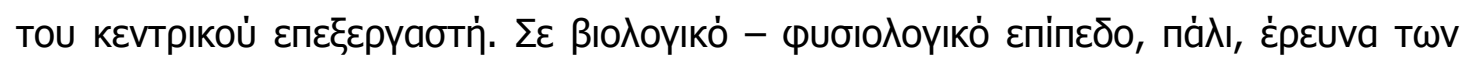

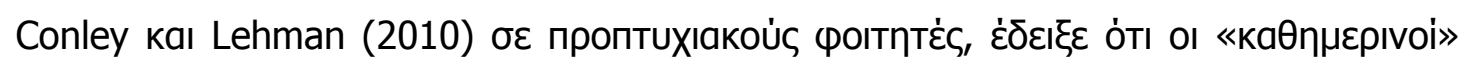

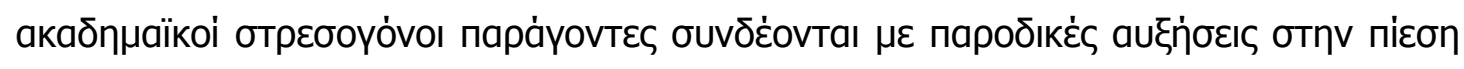

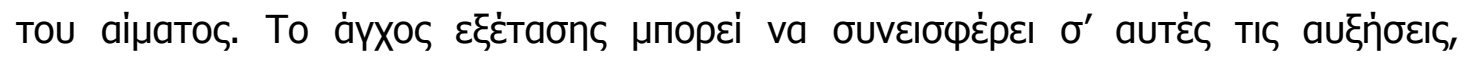

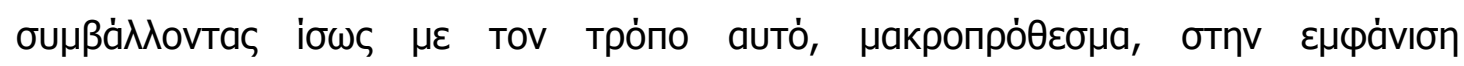

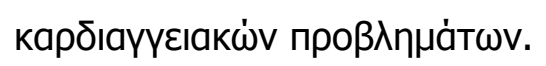

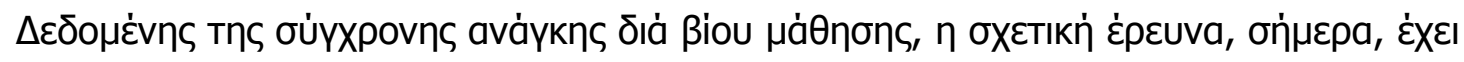

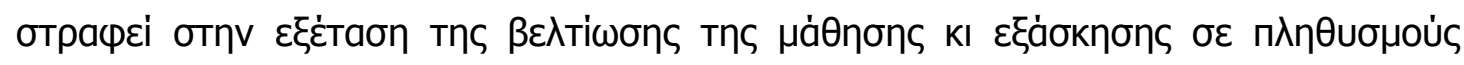

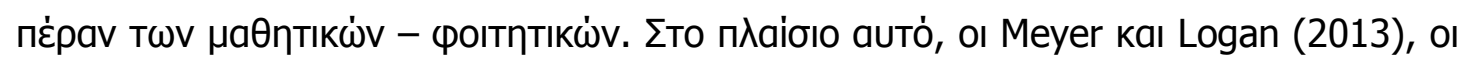

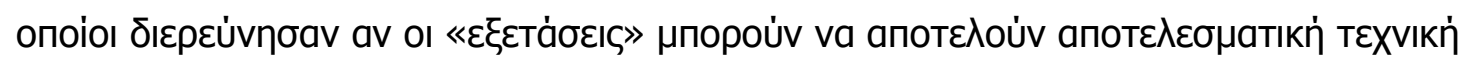

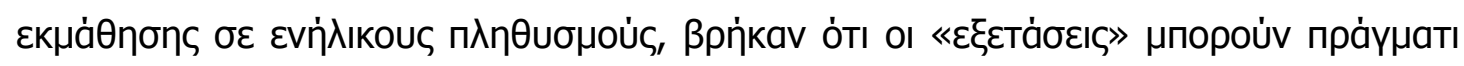

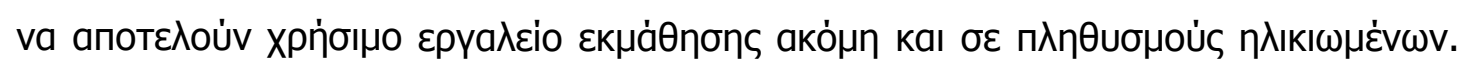




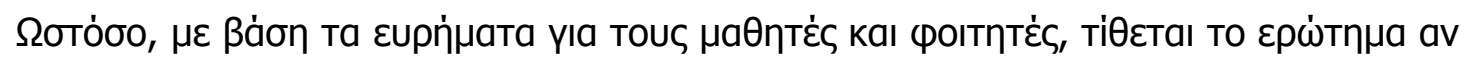

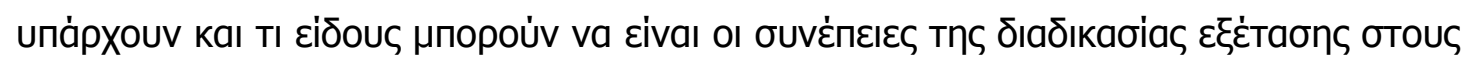

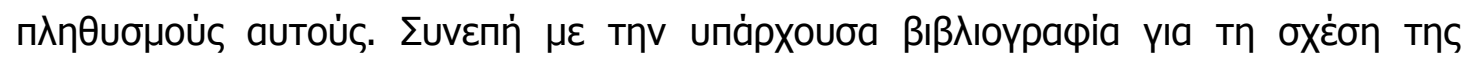

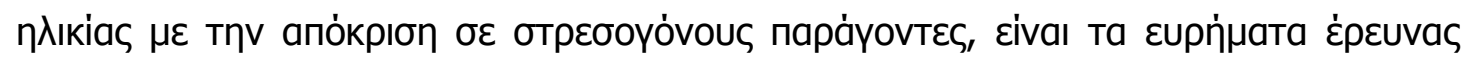

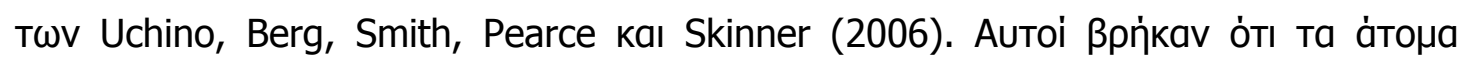

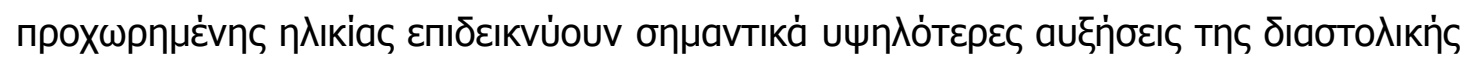

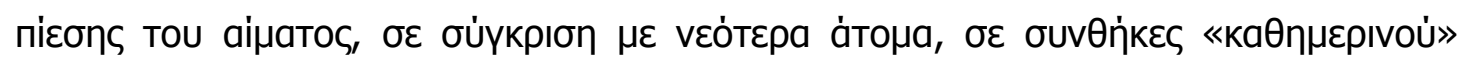

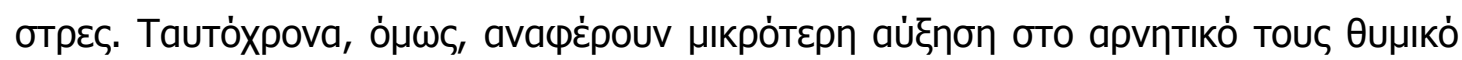

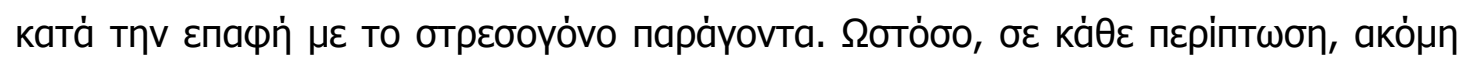

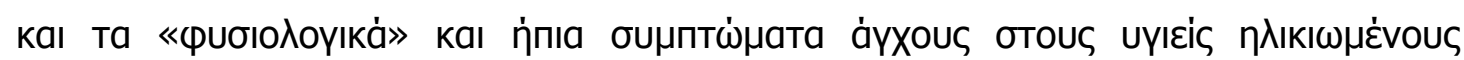

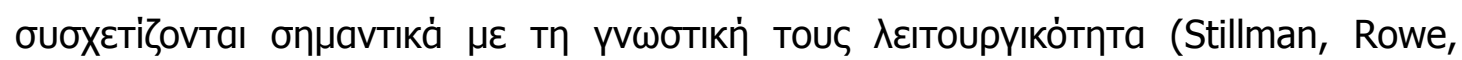

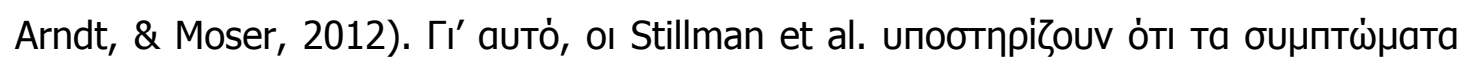

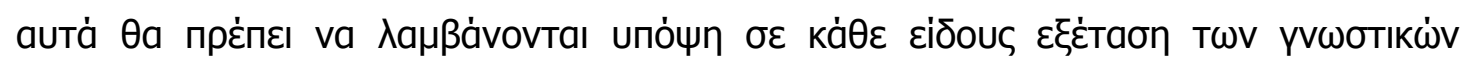

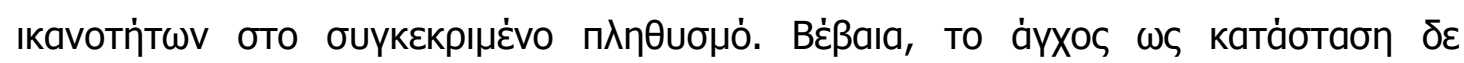

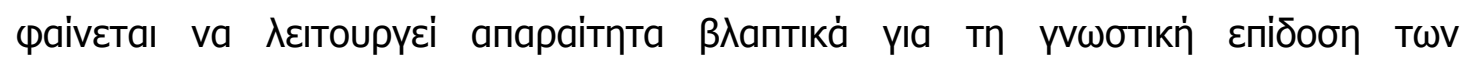

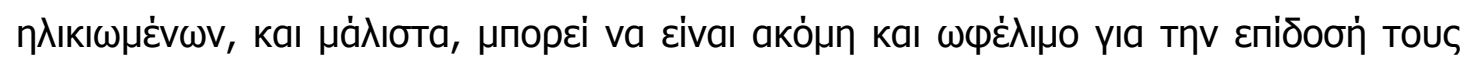

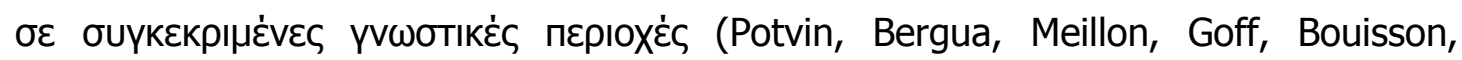

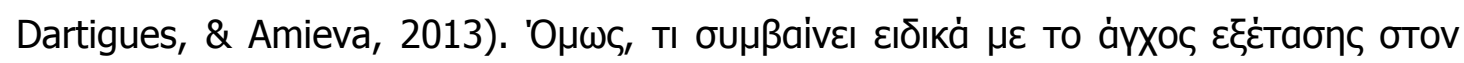

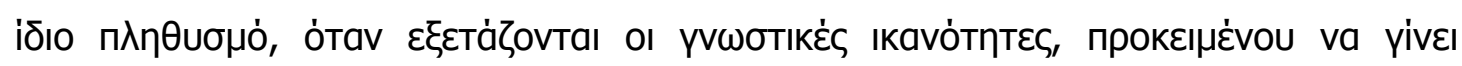

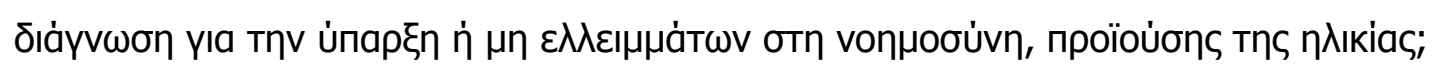

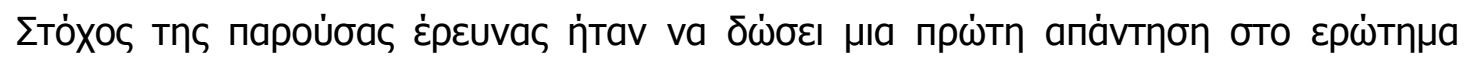

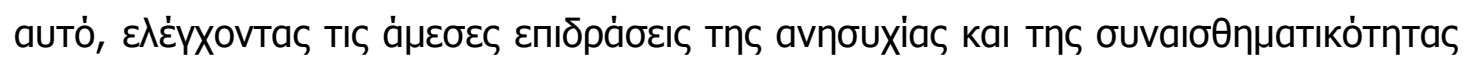

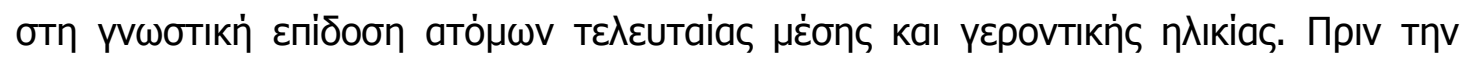

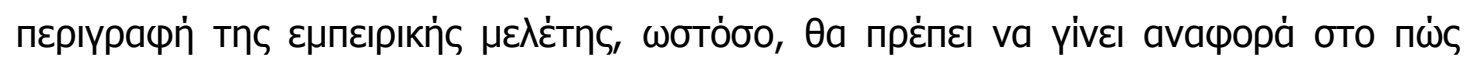

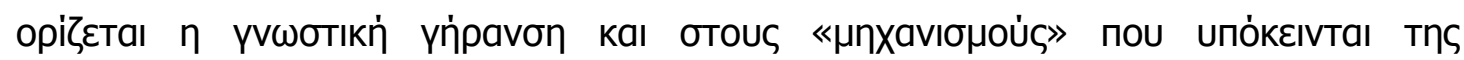

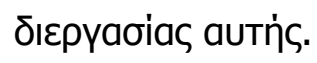

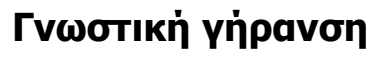

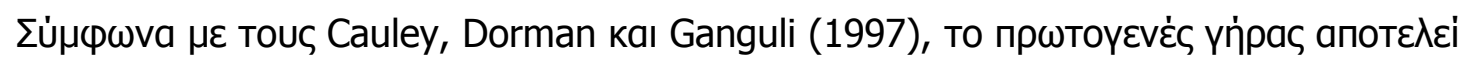

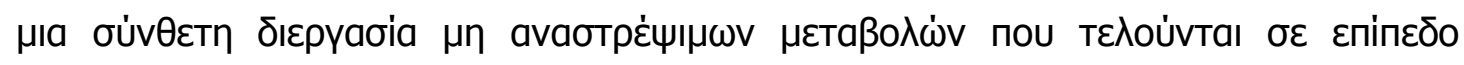

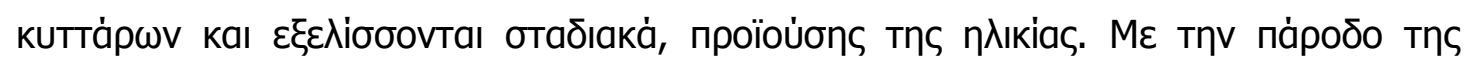

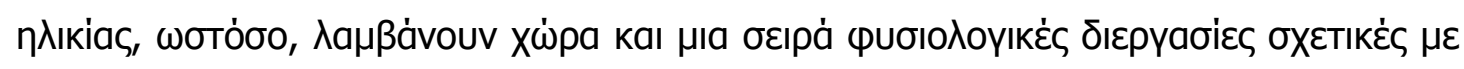

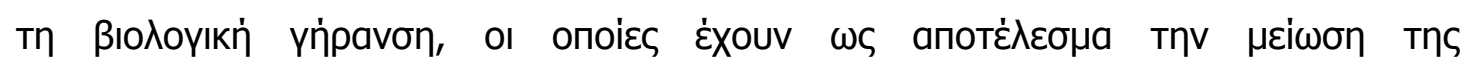

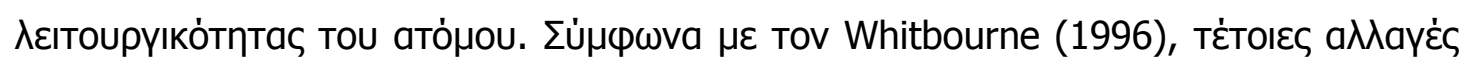




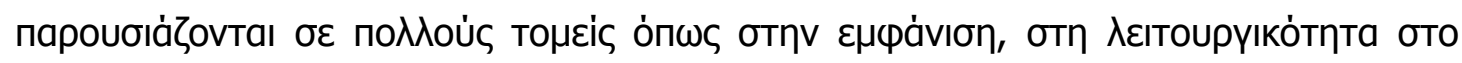

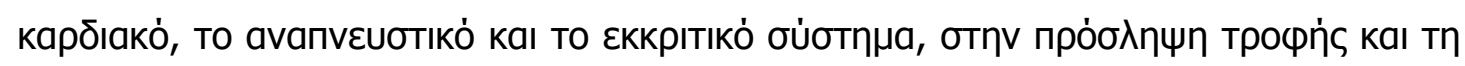

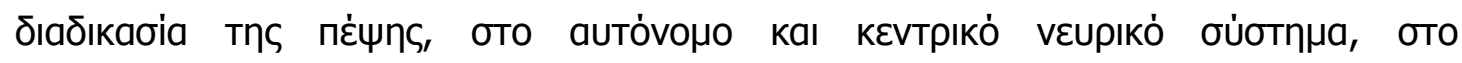

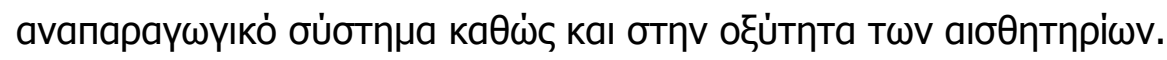

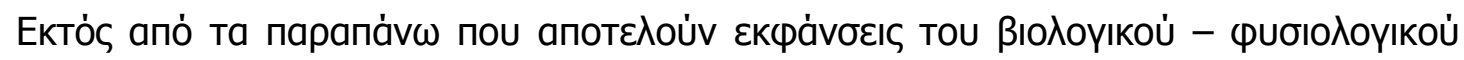

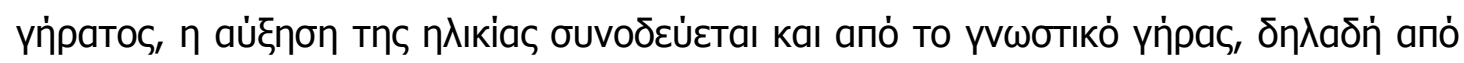

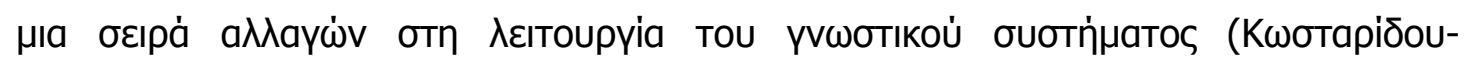

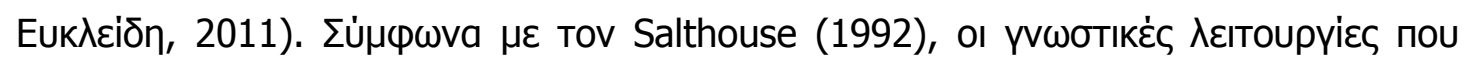

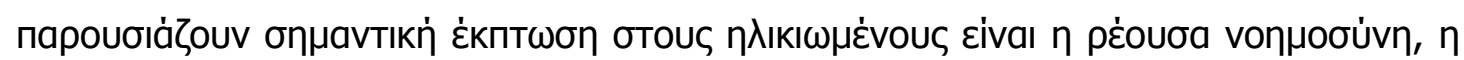

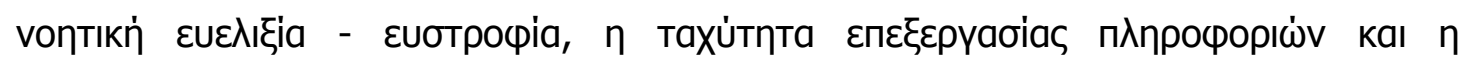

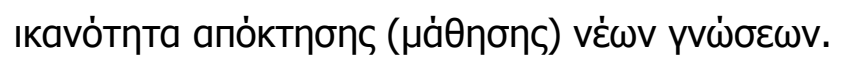

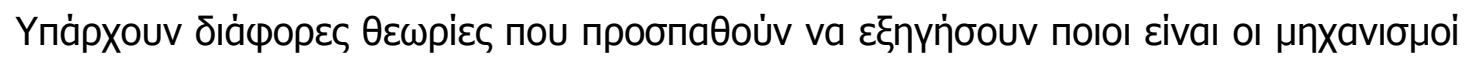

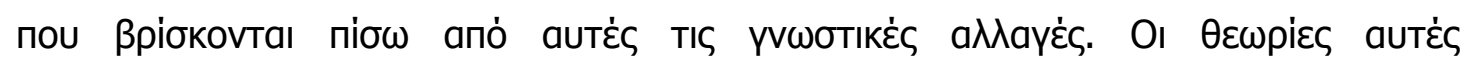

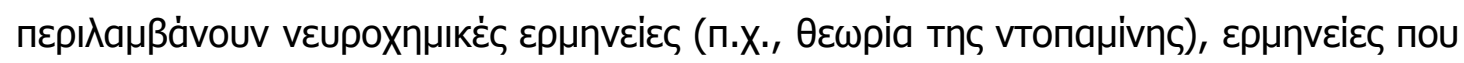

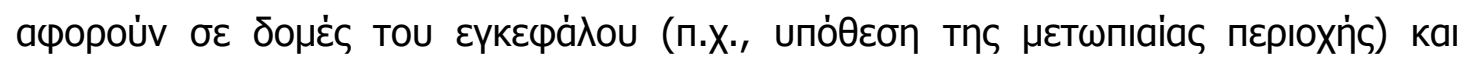

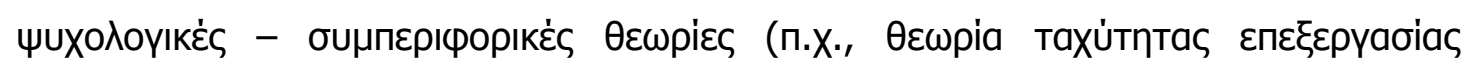

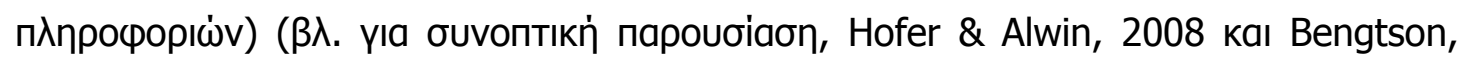

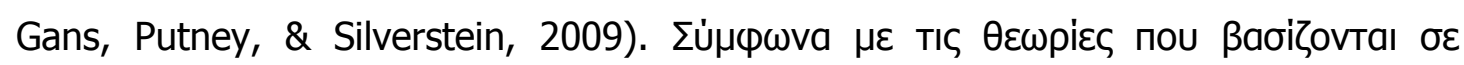

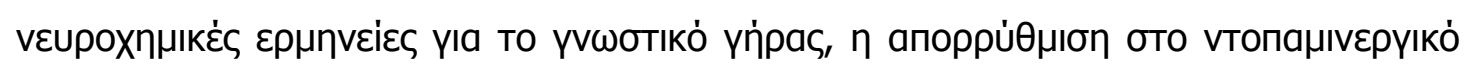

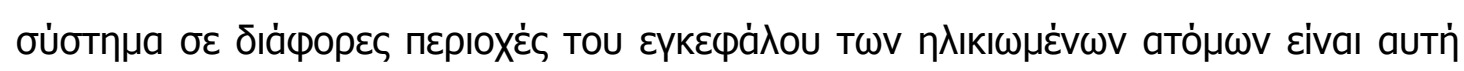

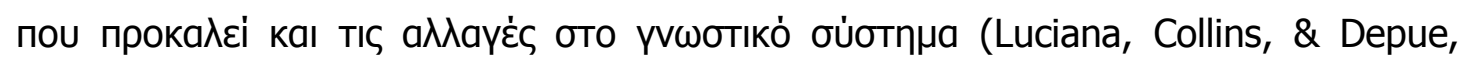

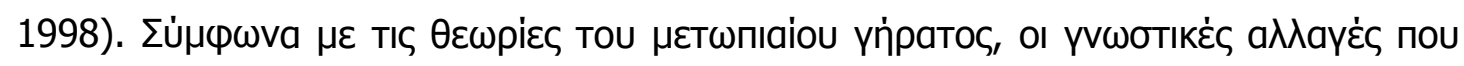

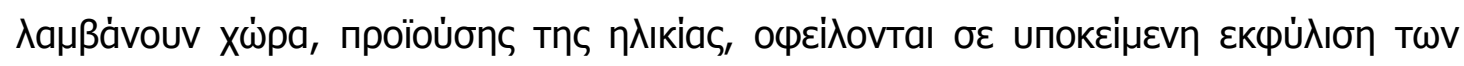

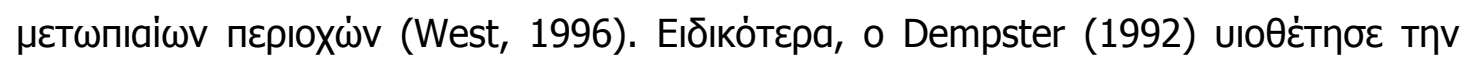

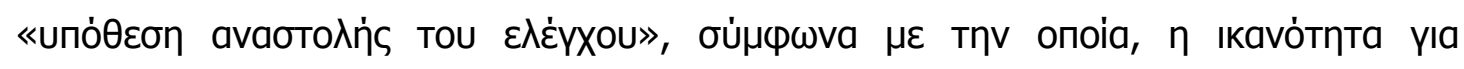

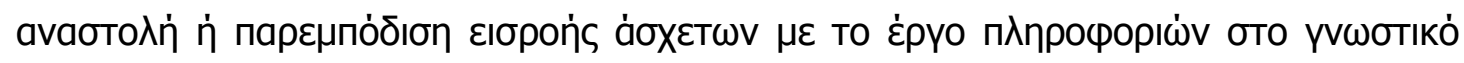

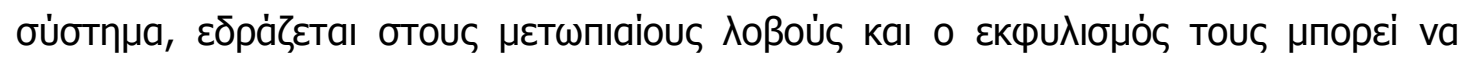

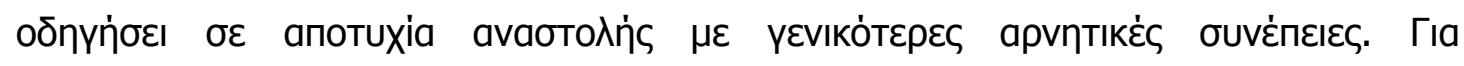

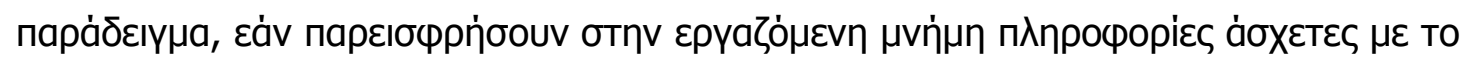

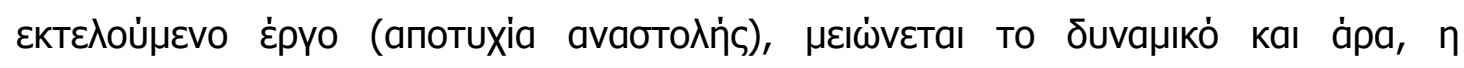

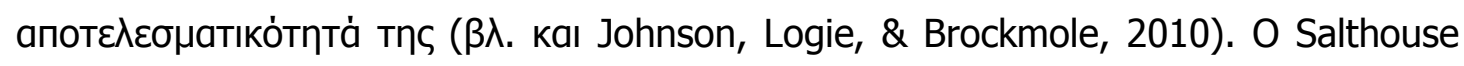

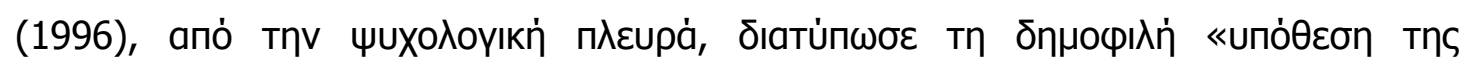

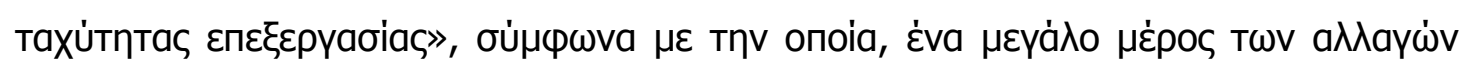




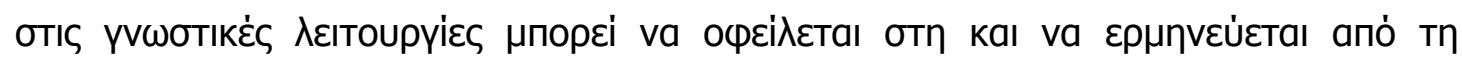

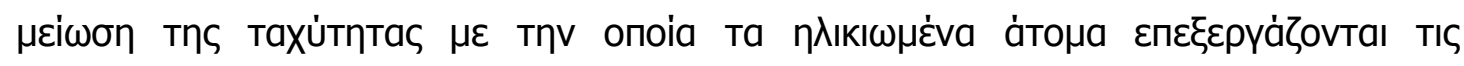

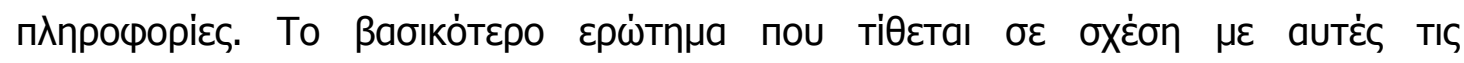

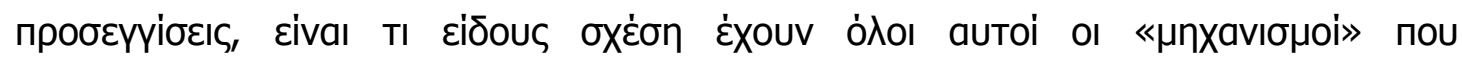

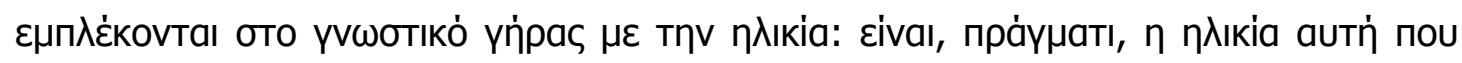

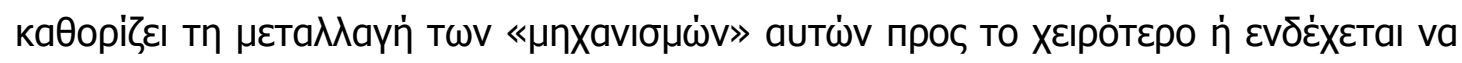

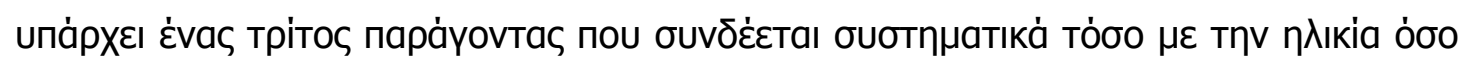

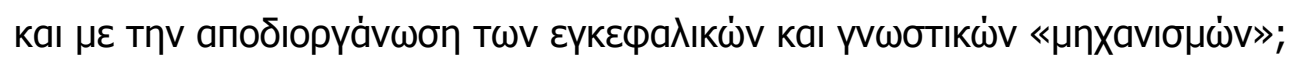

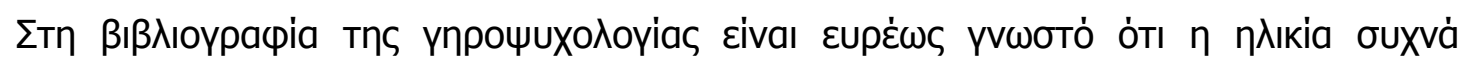

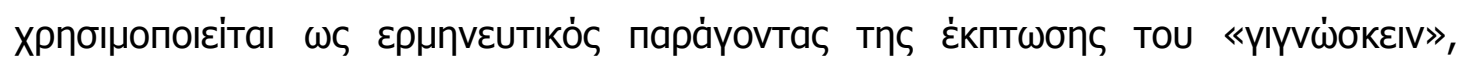

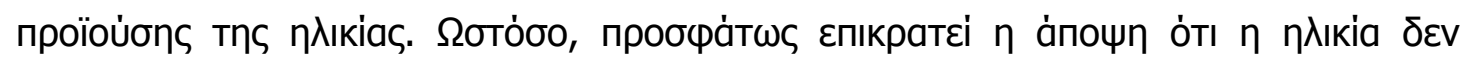

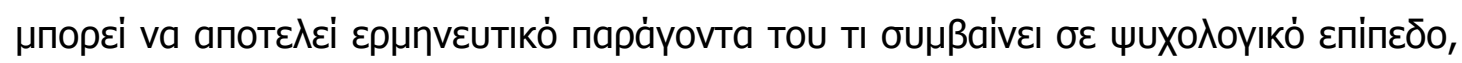

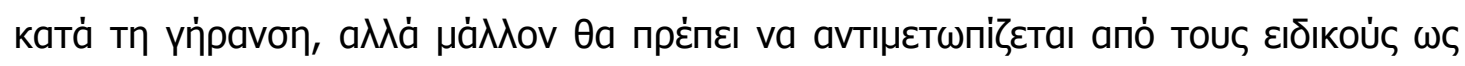

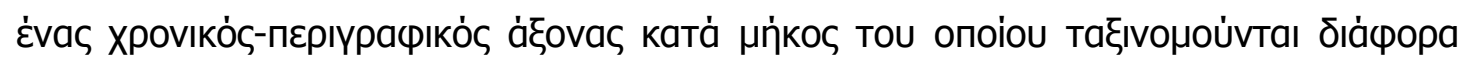

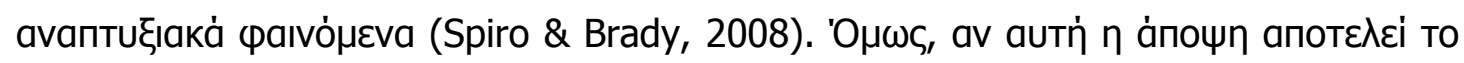

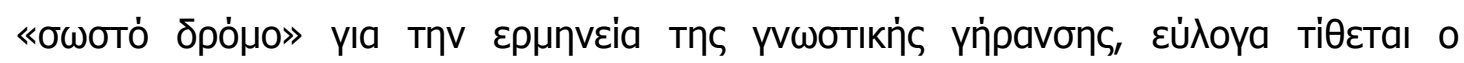

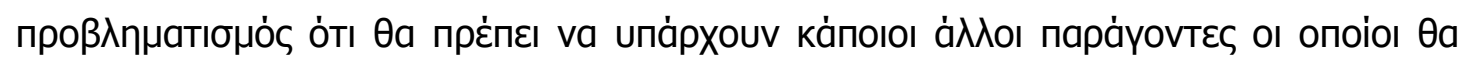

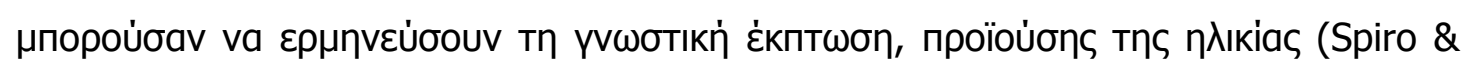

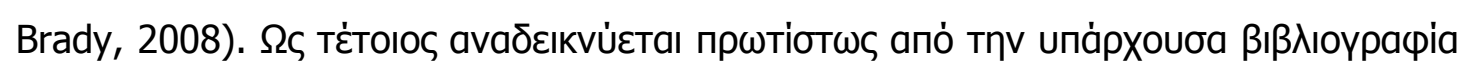

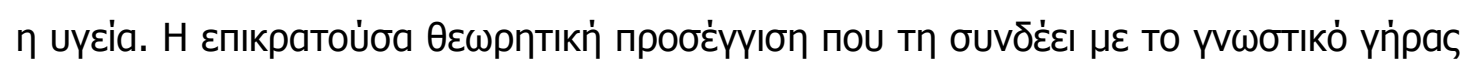

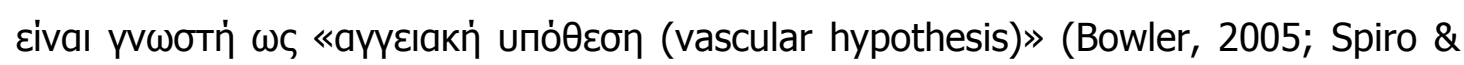

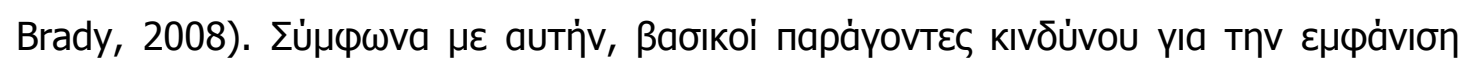

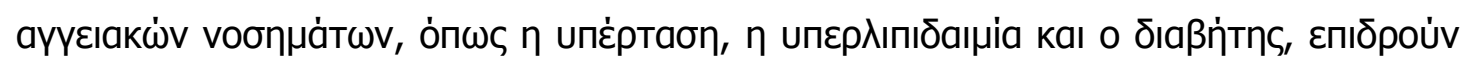

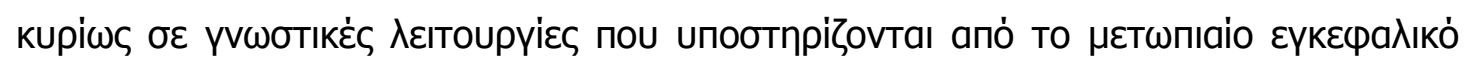

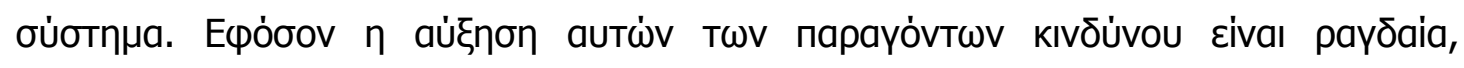

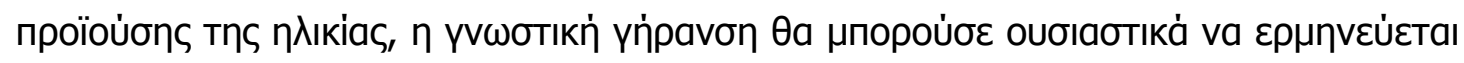

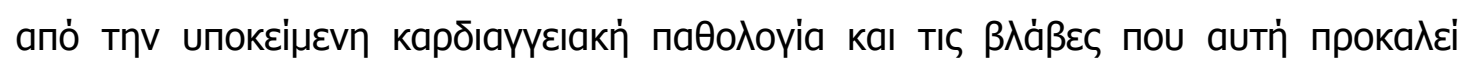

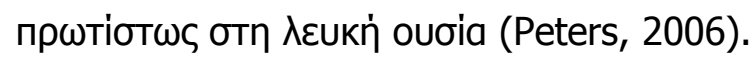

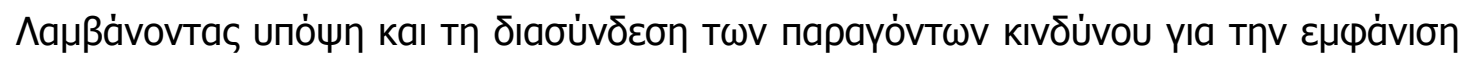

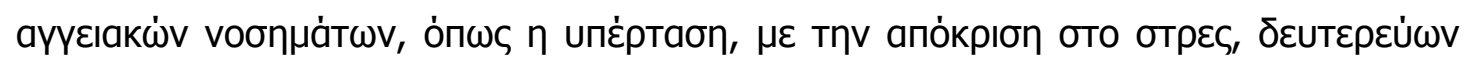

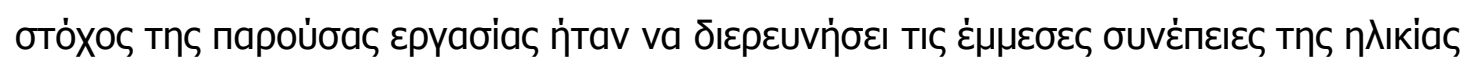

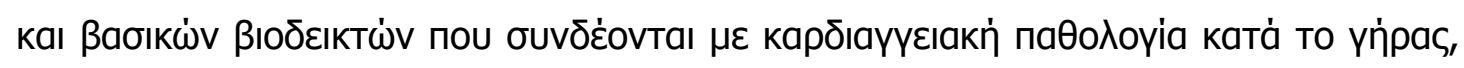

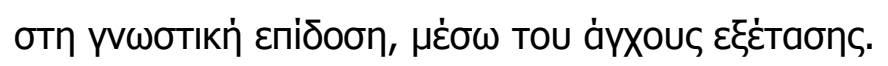




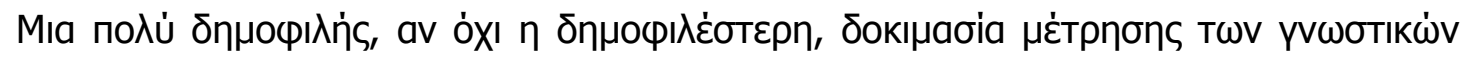

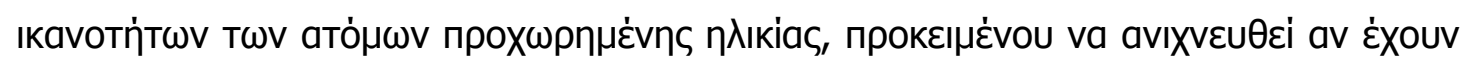

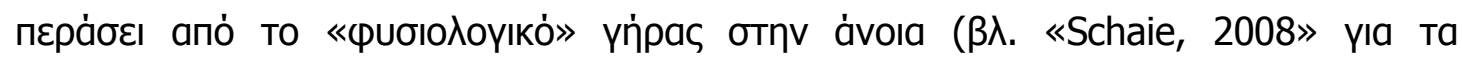

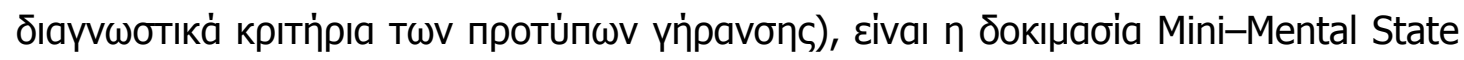

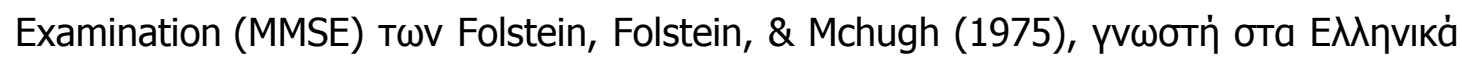

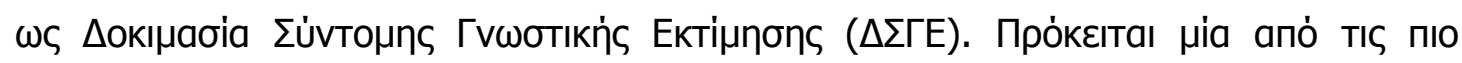

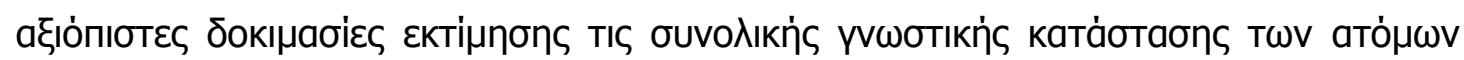

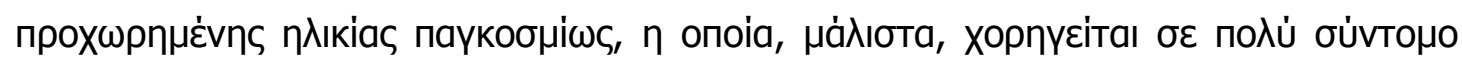

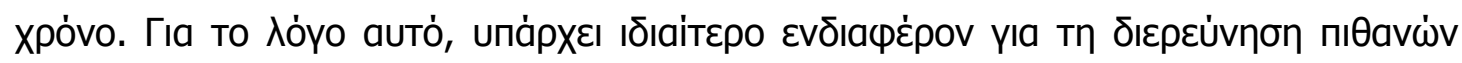

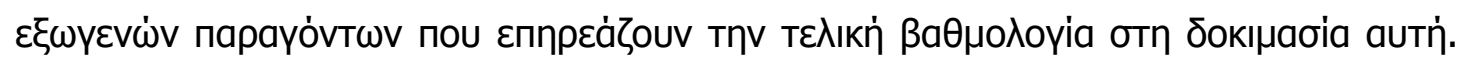

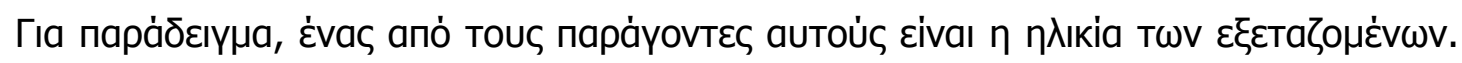
Oı Piccinin, Muniz-Terrera, Clouston, Reynolds, Thorvaldsson, Deary, Deeg,

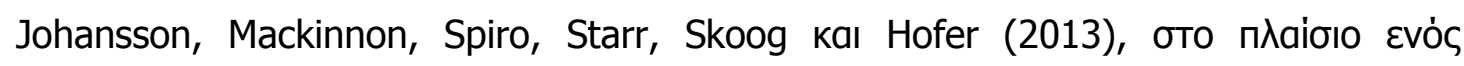

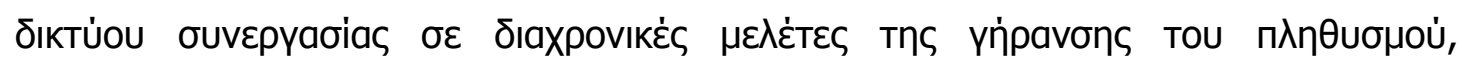

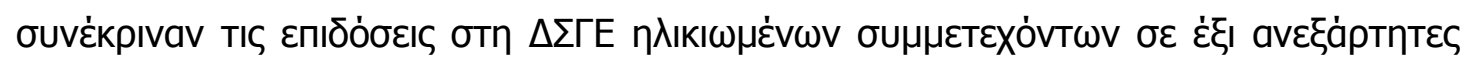

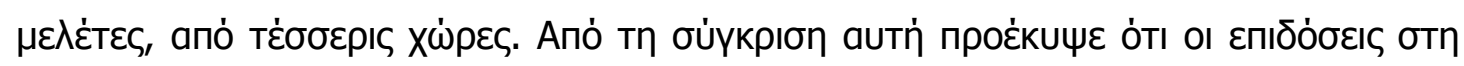

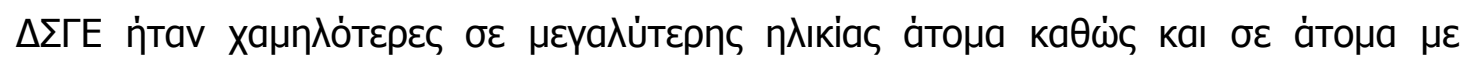

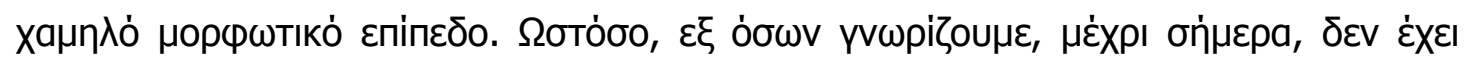

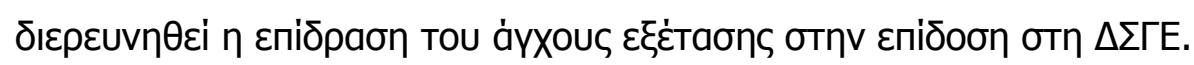

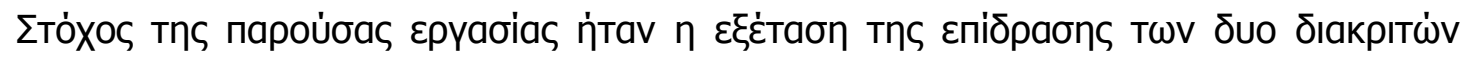

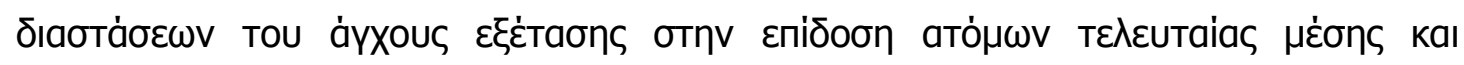

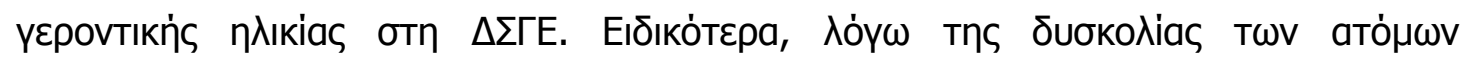

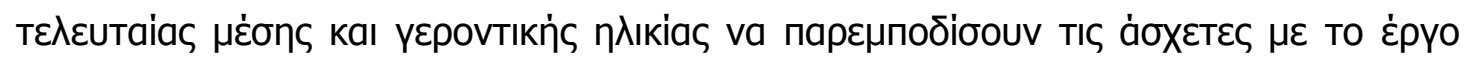

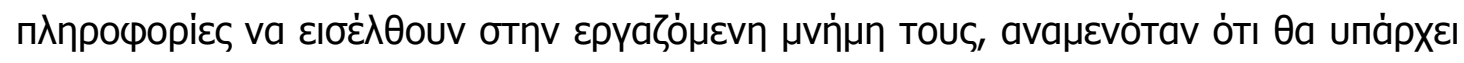

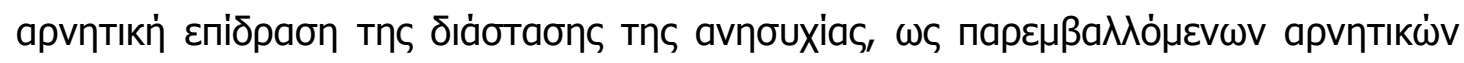

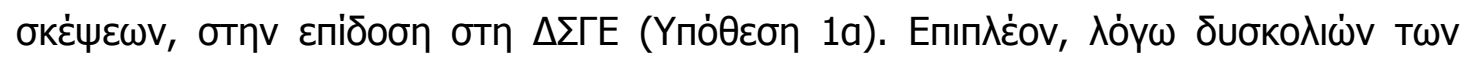

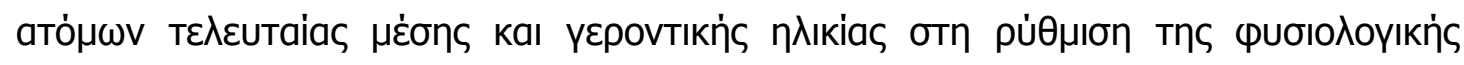

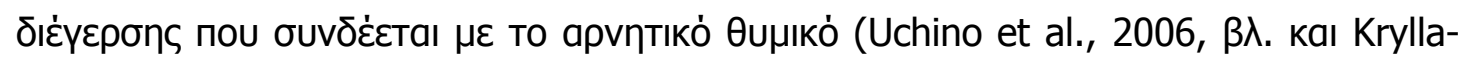

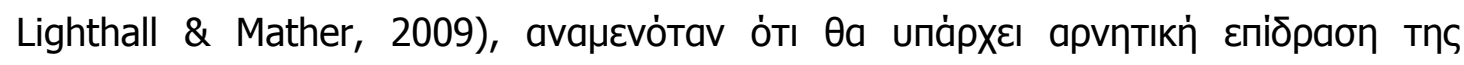

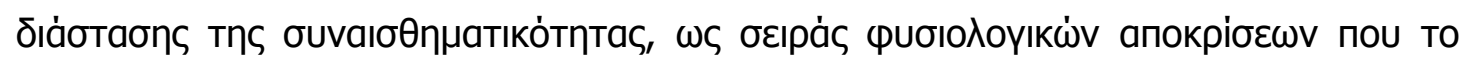

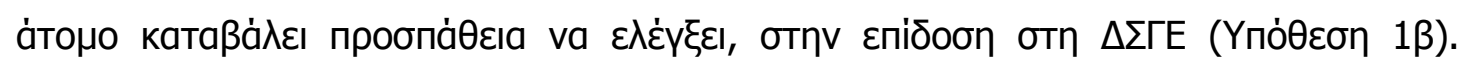

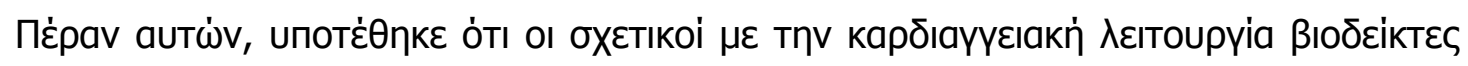

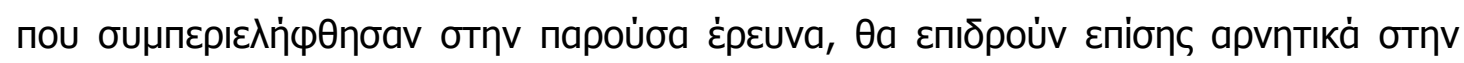




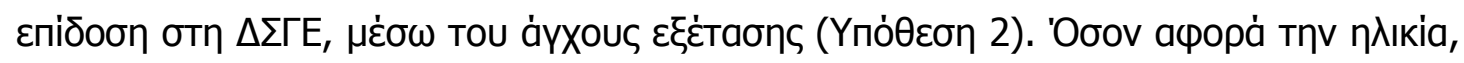

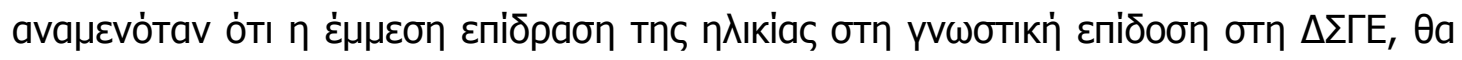

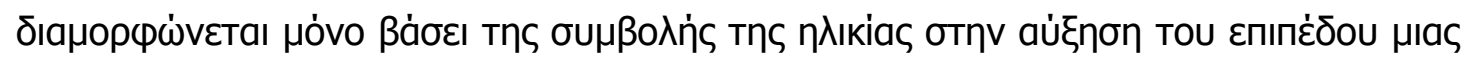

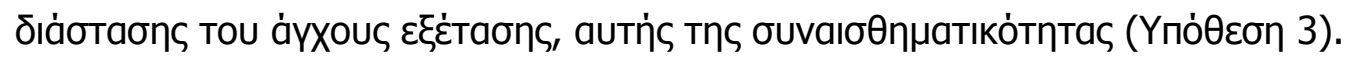




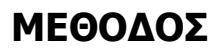

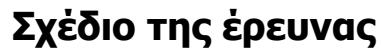

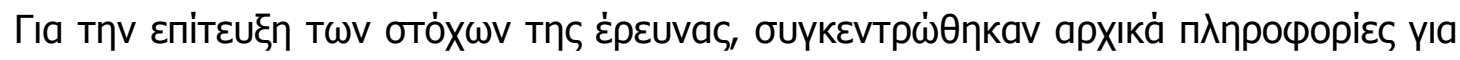

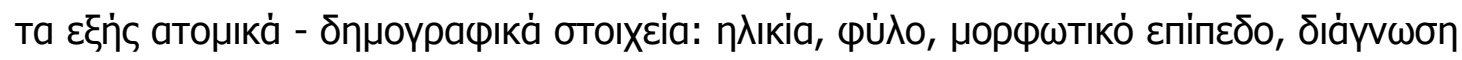

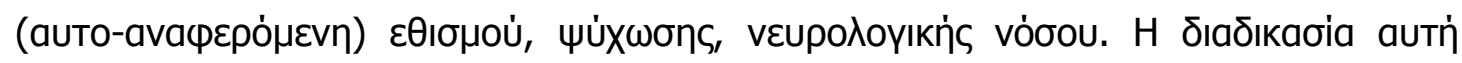

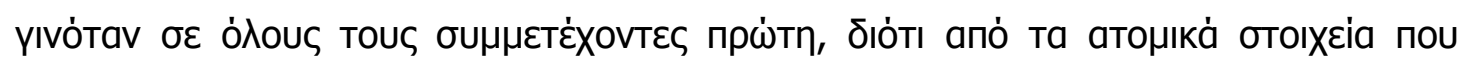

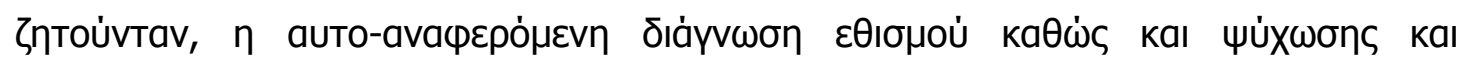

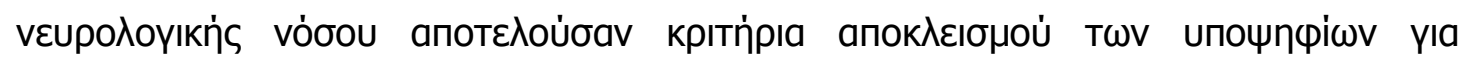

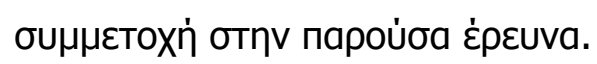

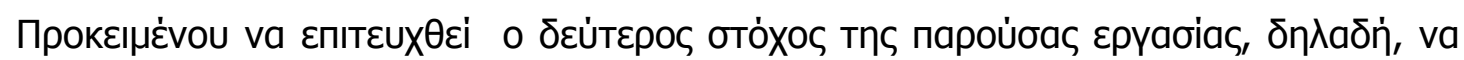

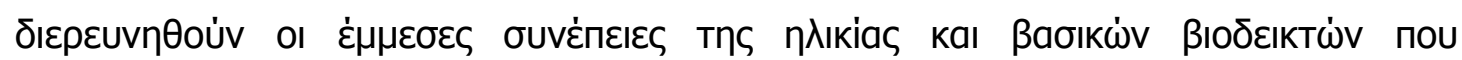

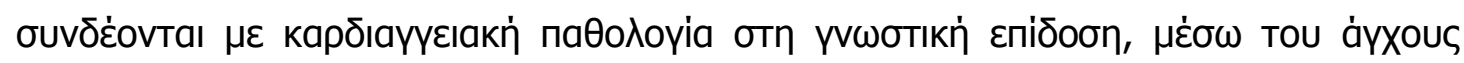

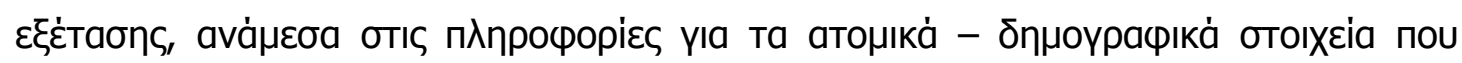

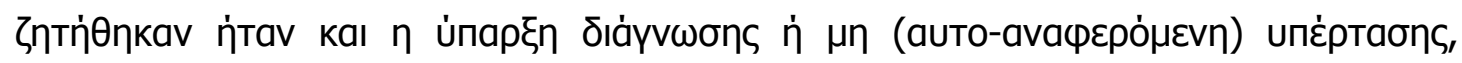

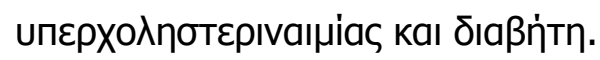

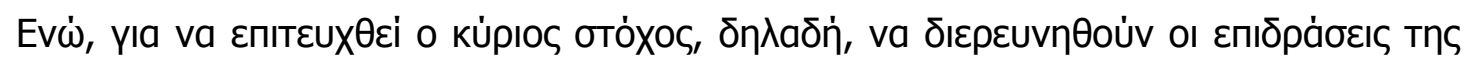

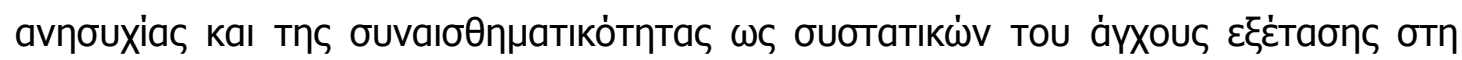

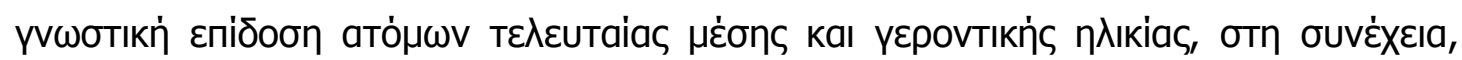

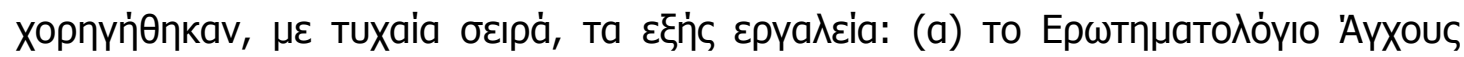

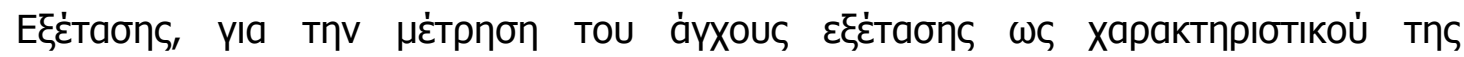

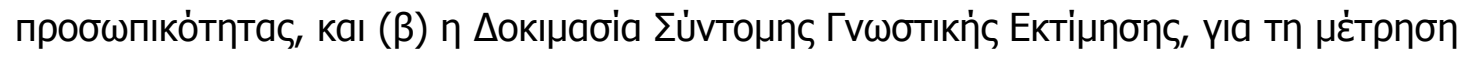

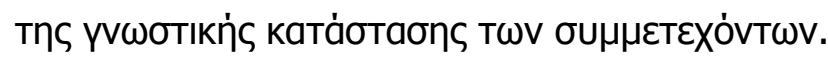

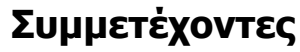

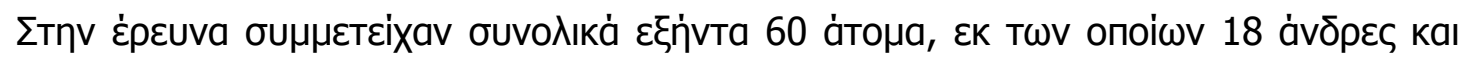

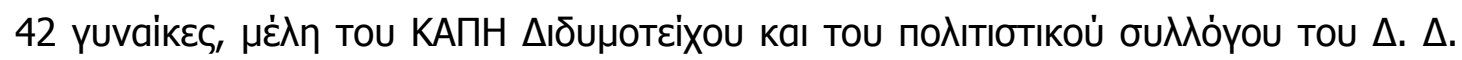

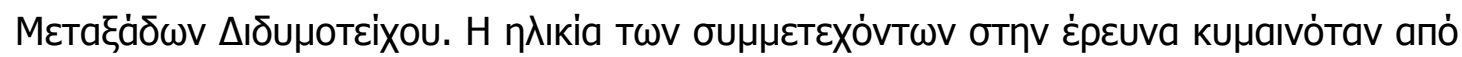

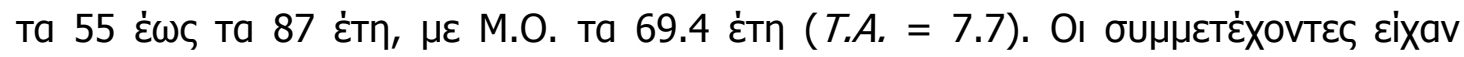

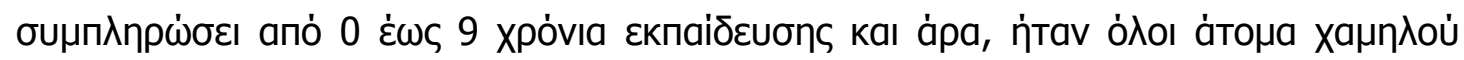

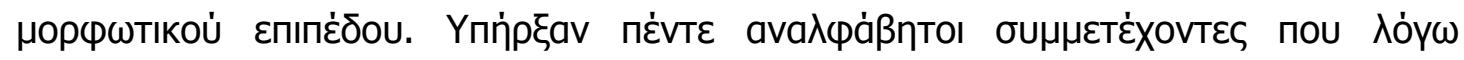

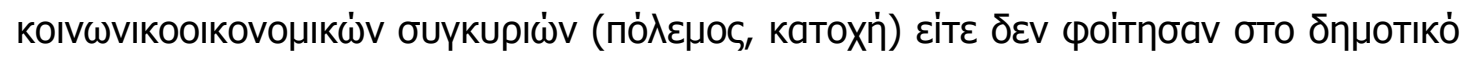

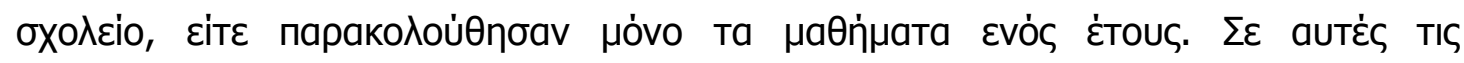

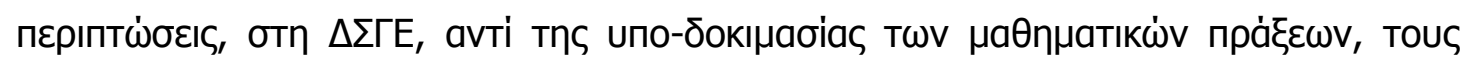

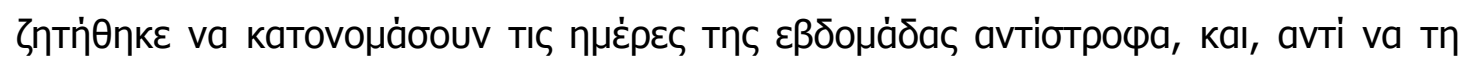

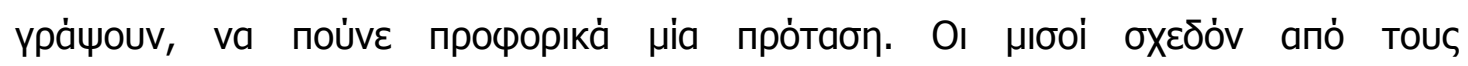




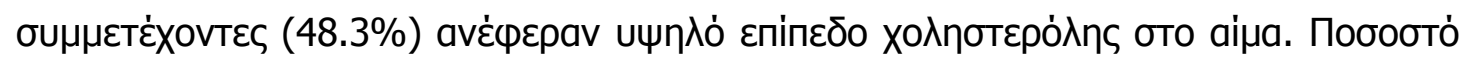

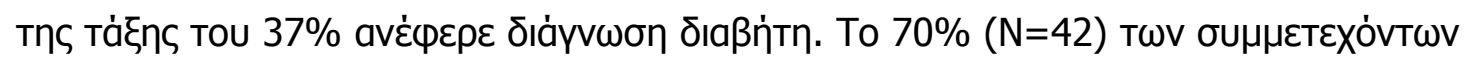

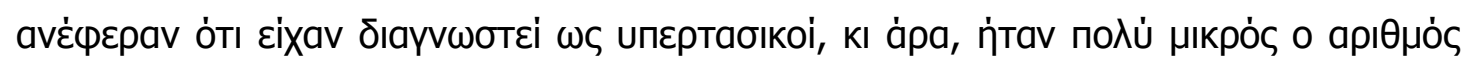

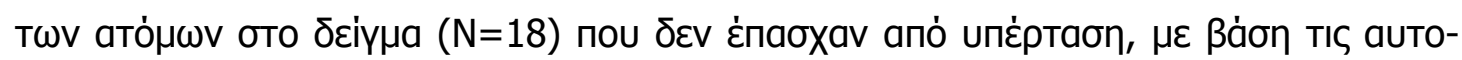

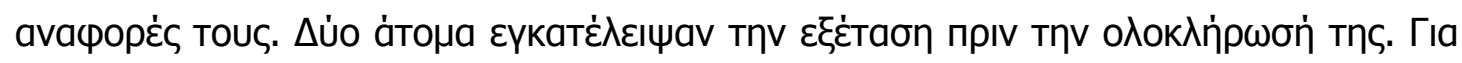

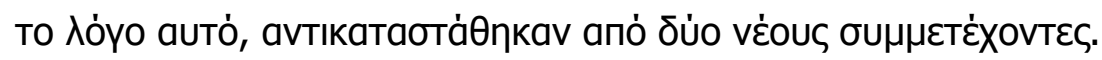

\section{'Epya}

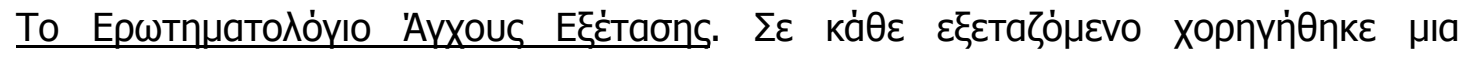

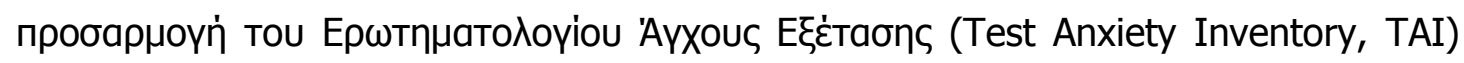
TwV Spielberger, Gonzales, Taylor, Anton, Algaze, Ross kal Westberry (1979). To

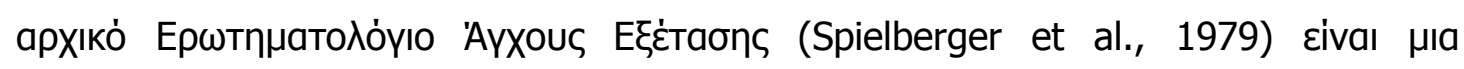

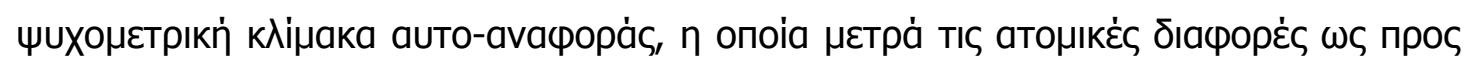

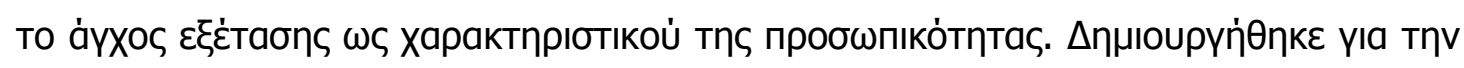

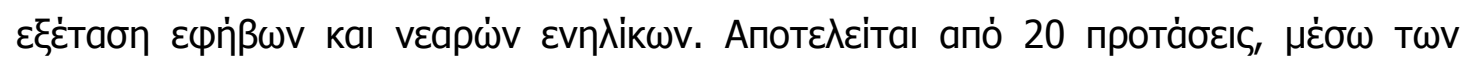

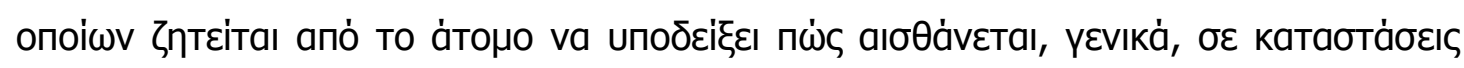

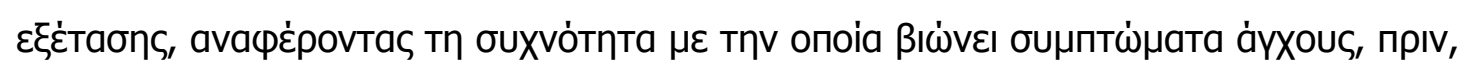

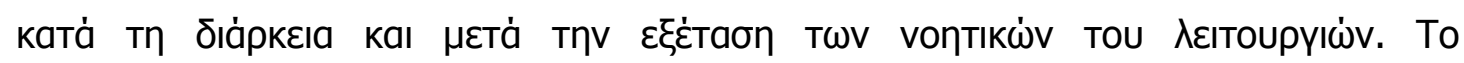

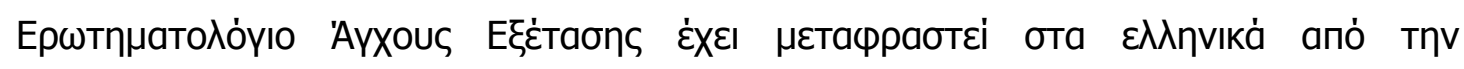

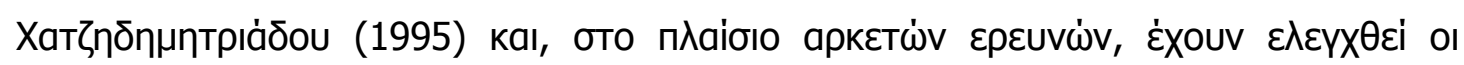

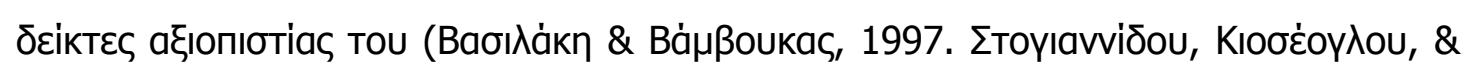

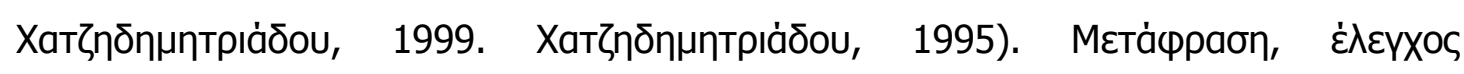

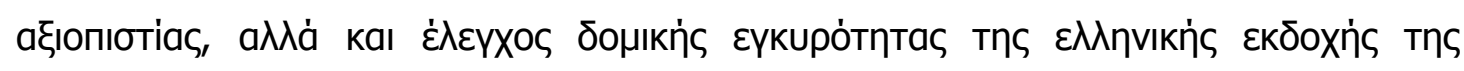

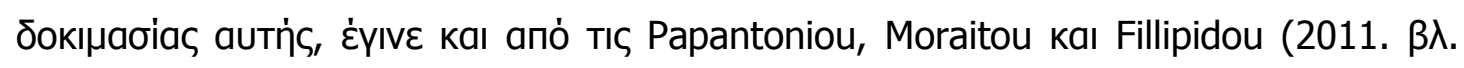

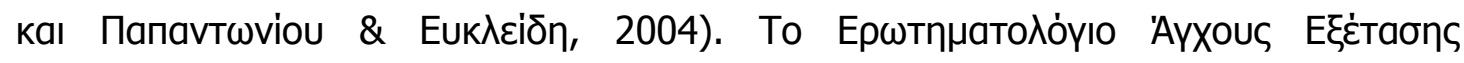

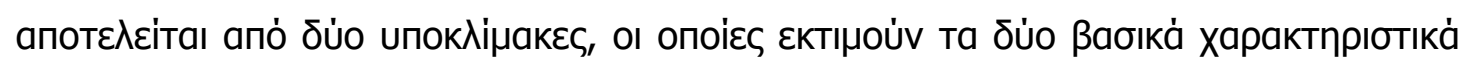

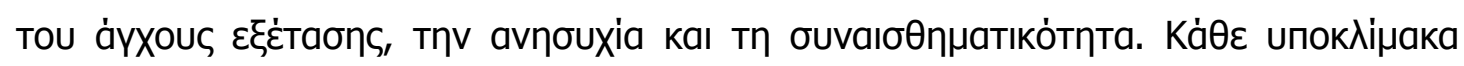

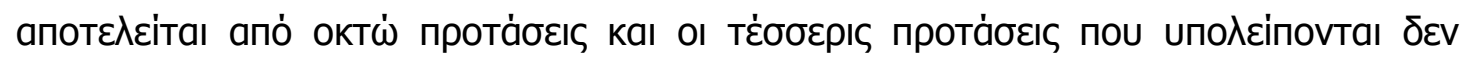

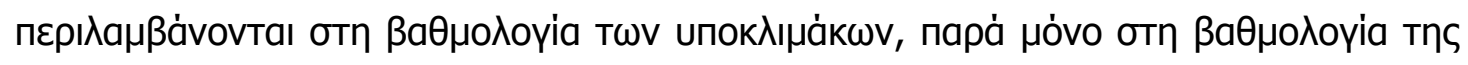

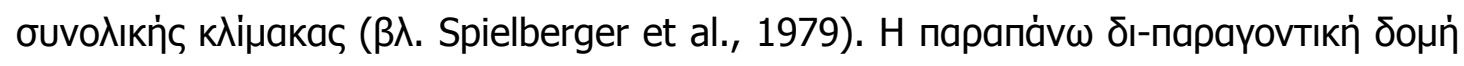

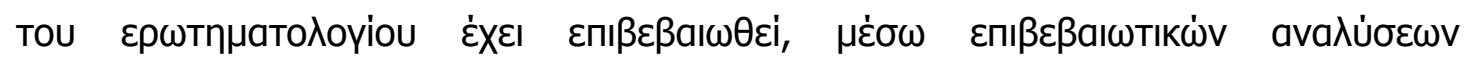

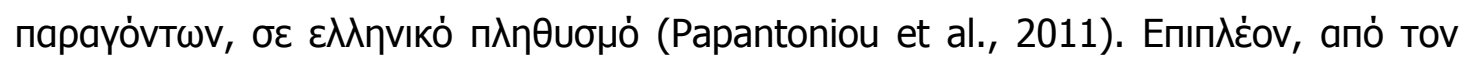

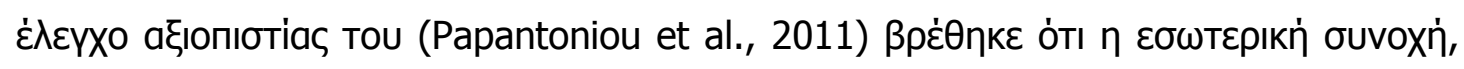

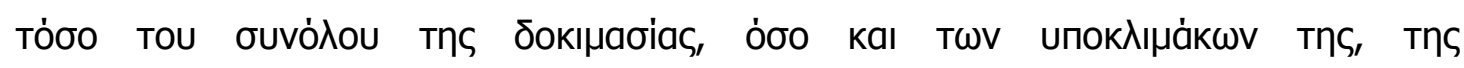




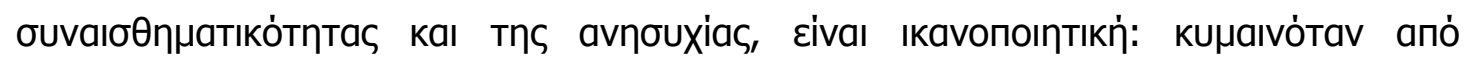

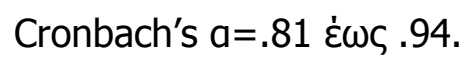

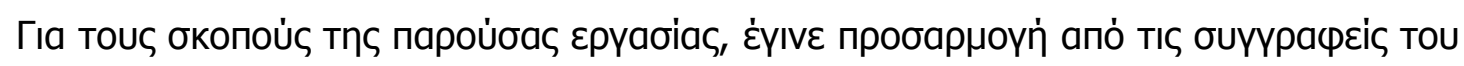

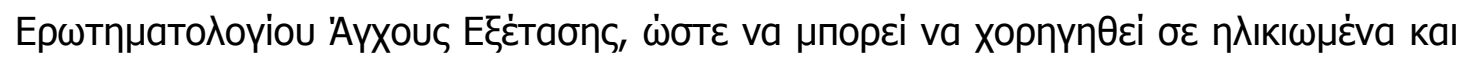

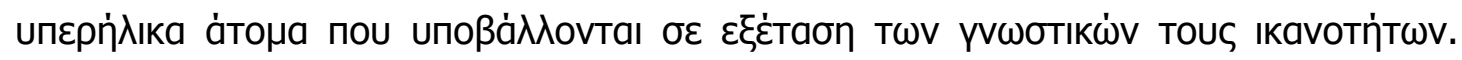

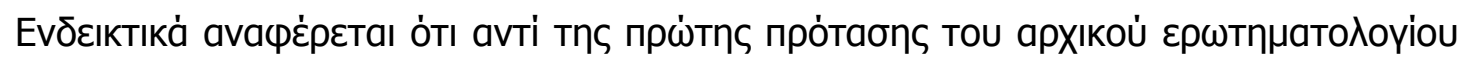

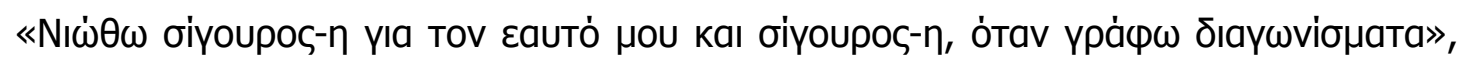

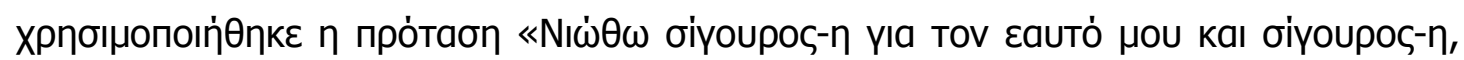

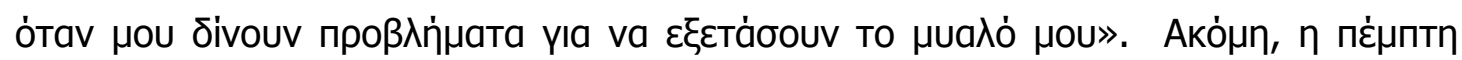

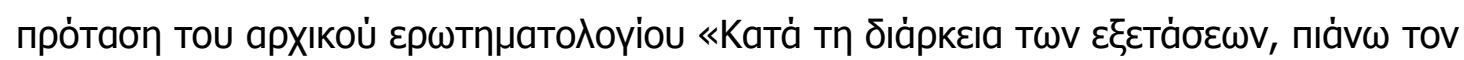

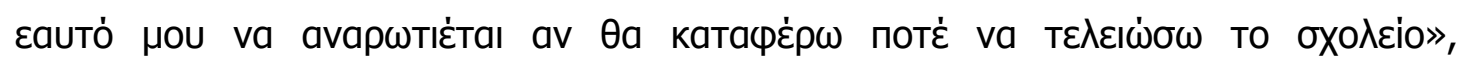

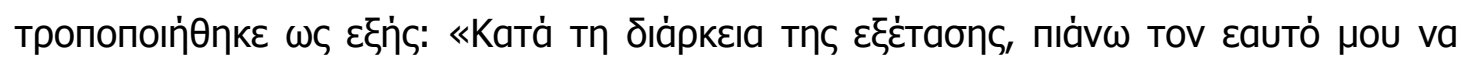

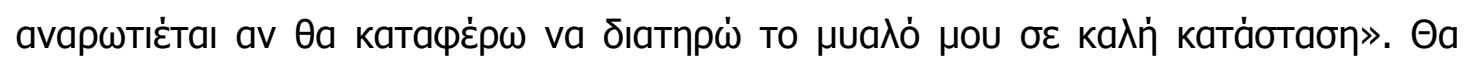

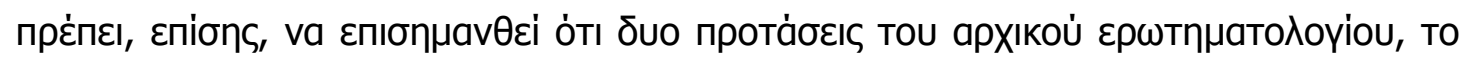

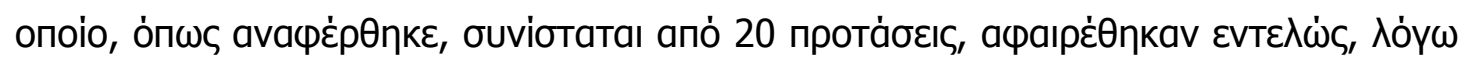

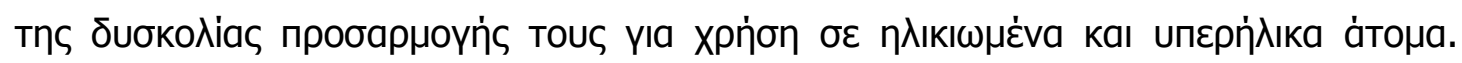

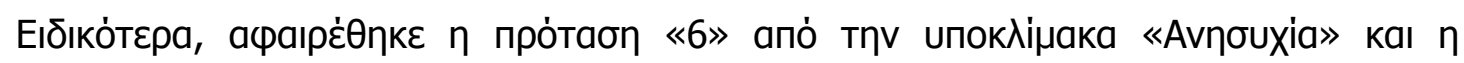

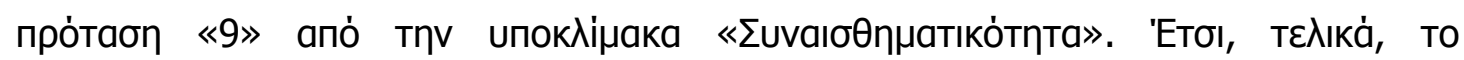

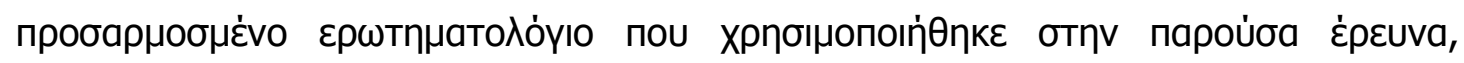

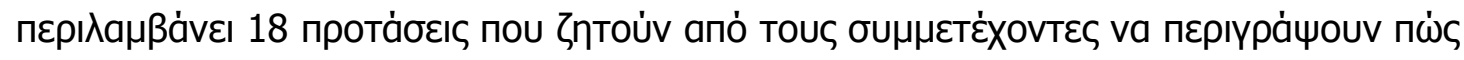

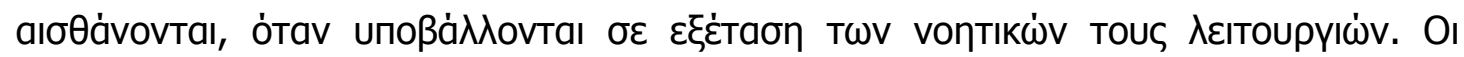

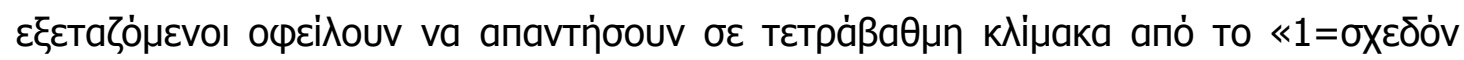

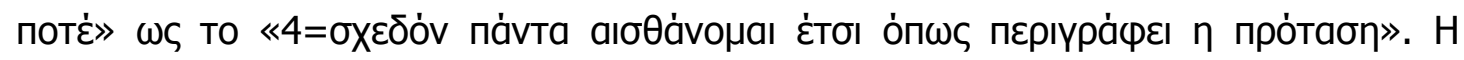

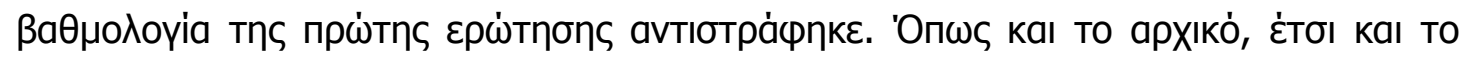

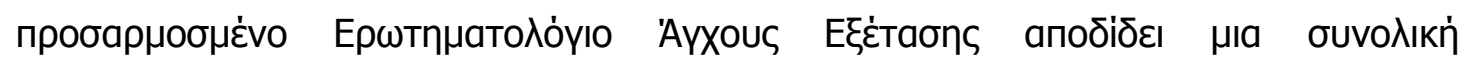

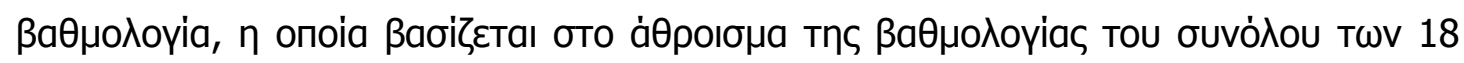

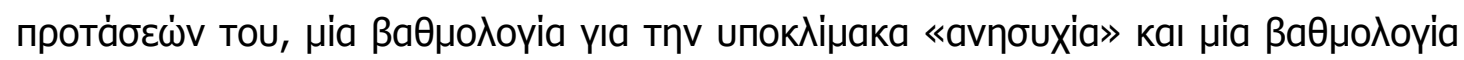

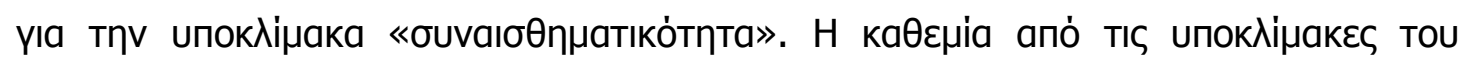

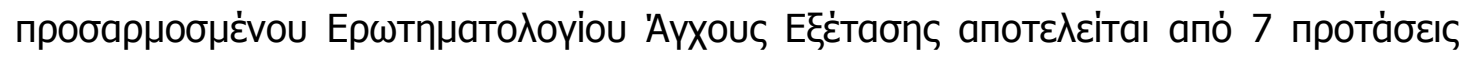

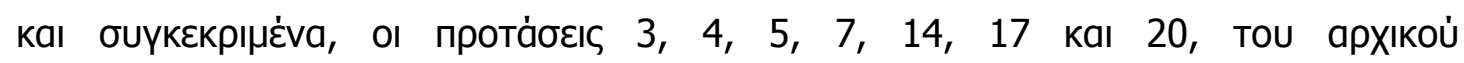

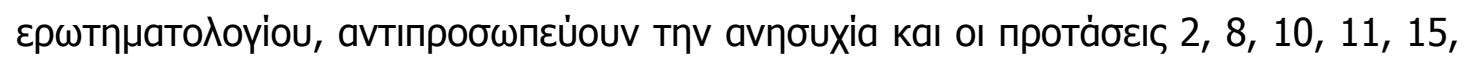

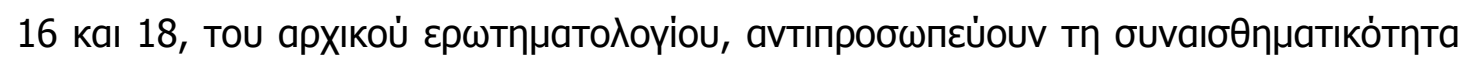

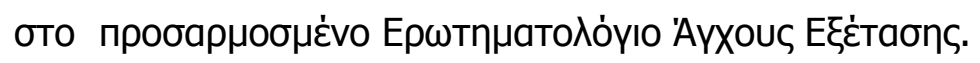




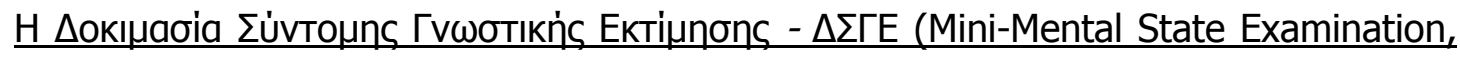

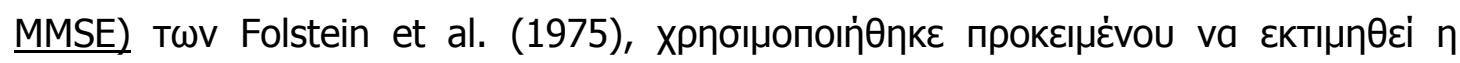

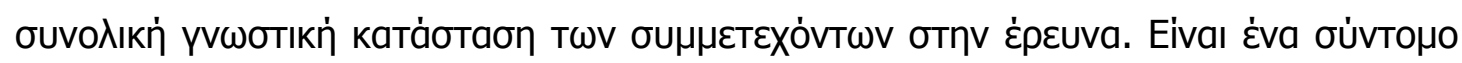

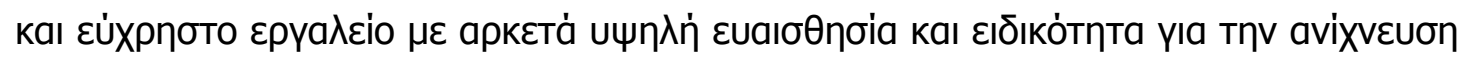

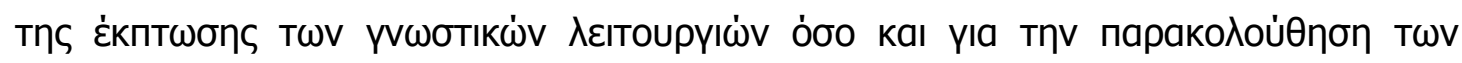

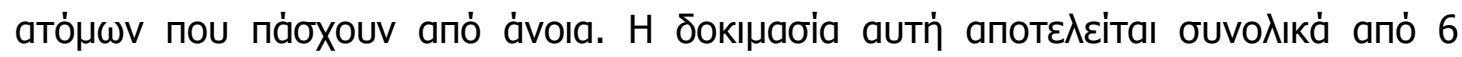

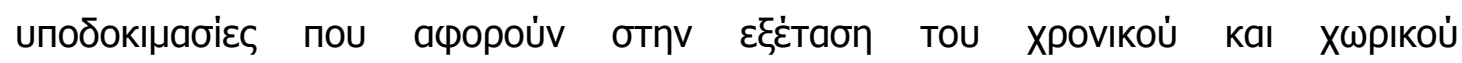

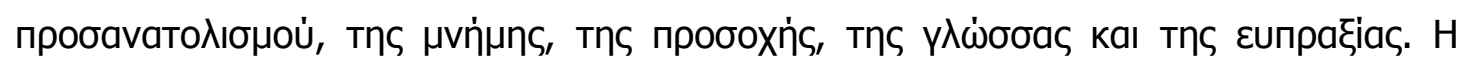

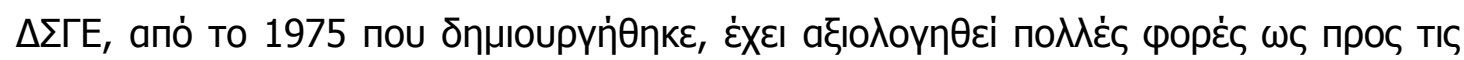

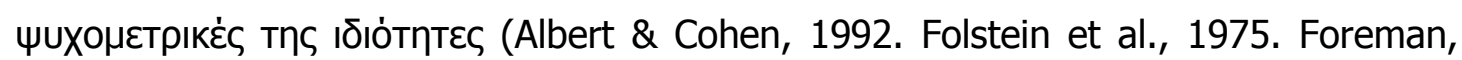
1987. Holzer, Tischler, Leaf, \& Myers, 1984. Hopp, Dixon, Grut, \& Backman, 1997. Morales, Flowers, Gutierrez, Kleinman, \& Tenesi, 2006. Shigemori, Ohgi, Okuyama,

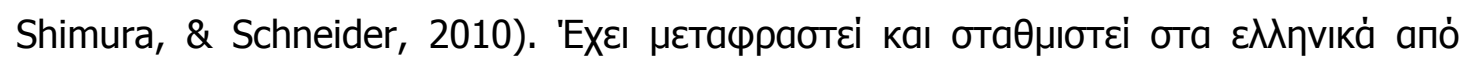

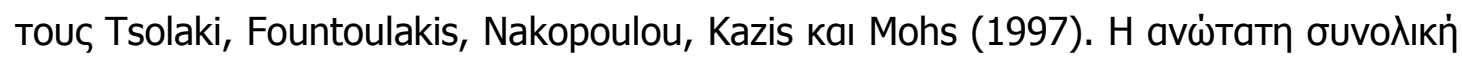

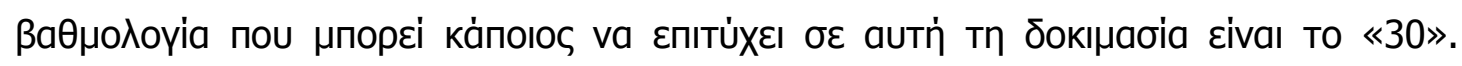

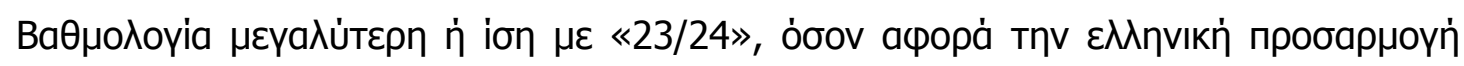

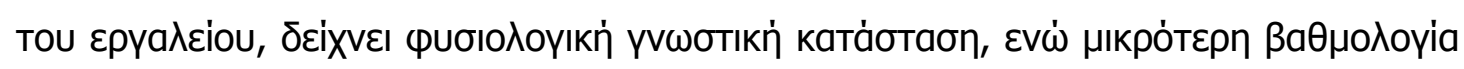

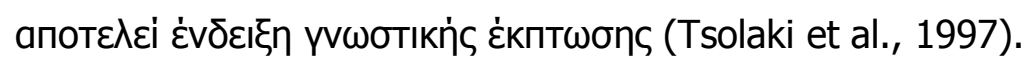

\section{Aıаסıкабia}

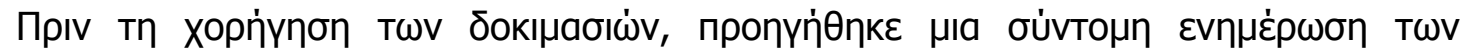

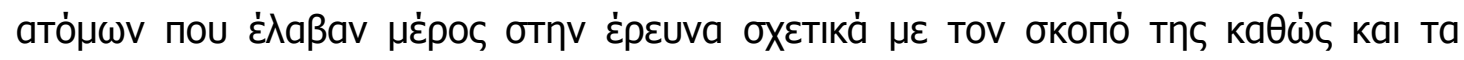
ठІкаı

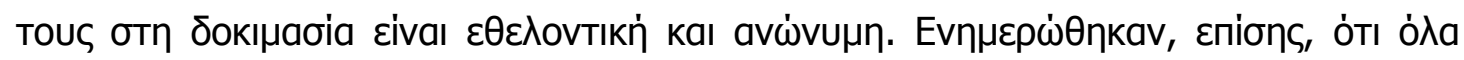

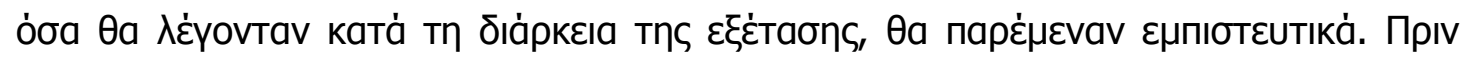

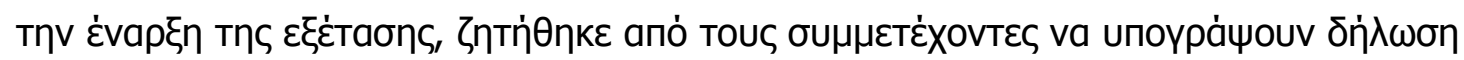

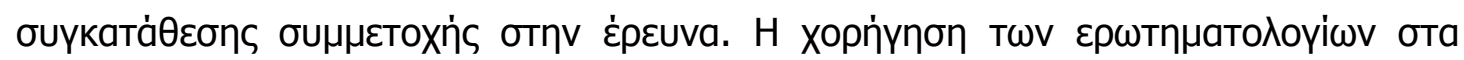

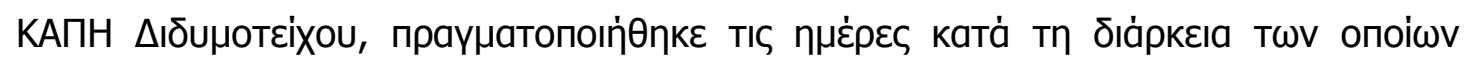

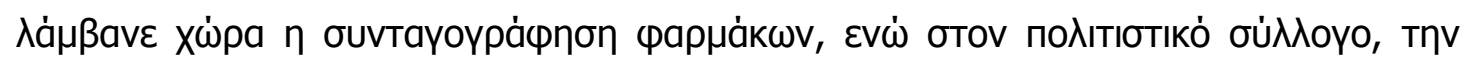

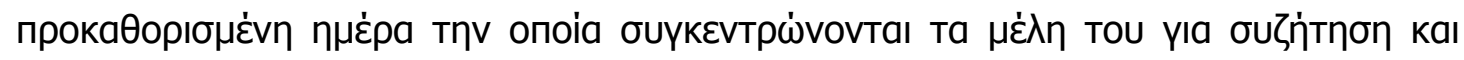

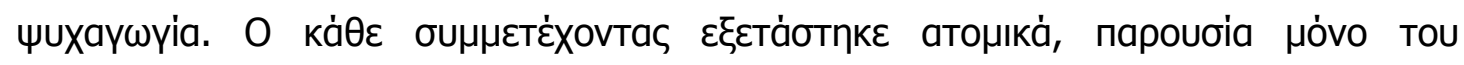

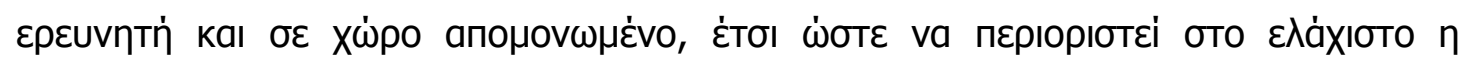

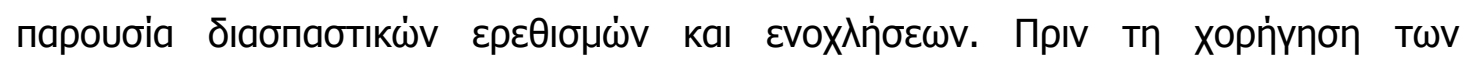

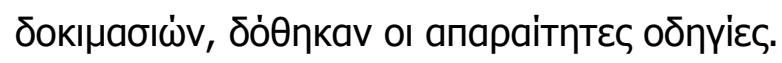




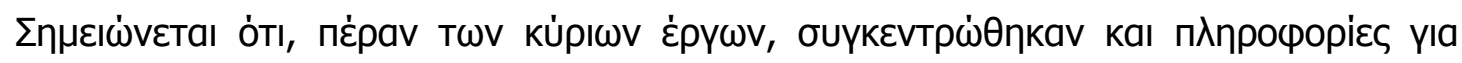

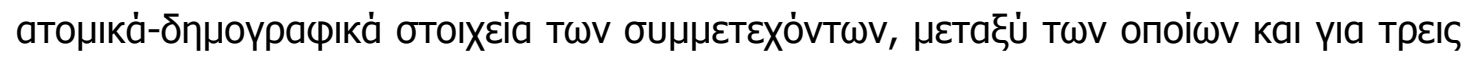

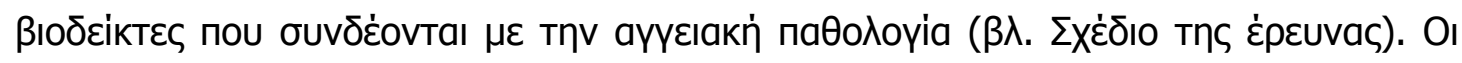

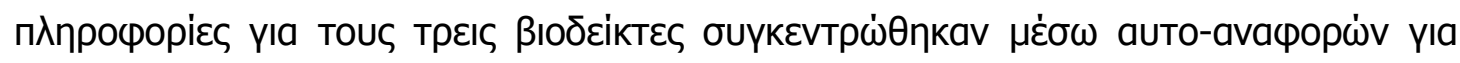

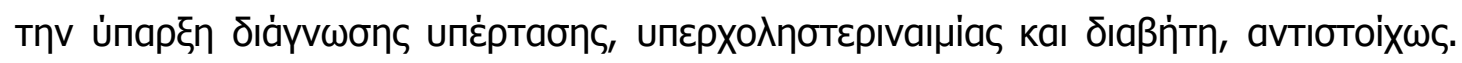

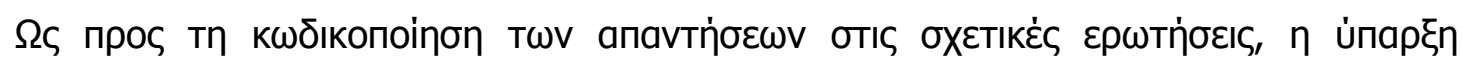

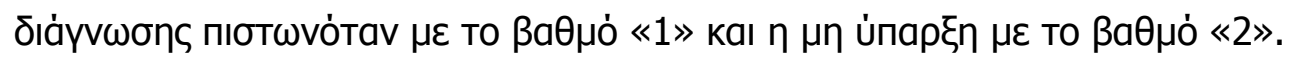

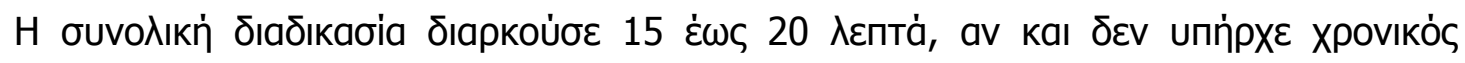

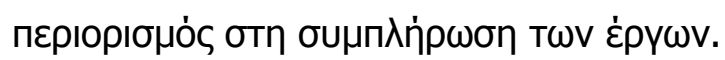

\section{AПOTEAEรMATA}

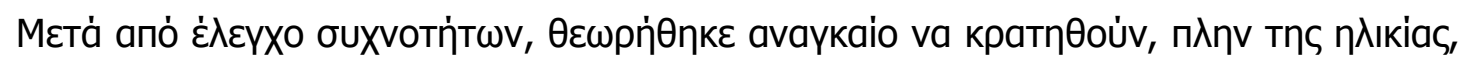

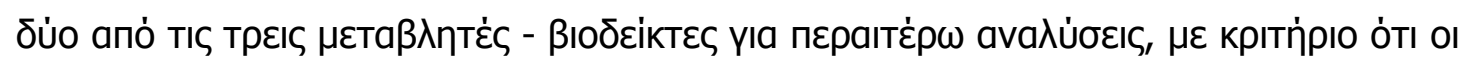

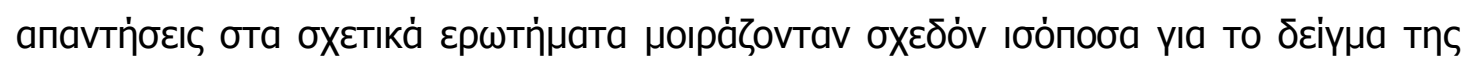

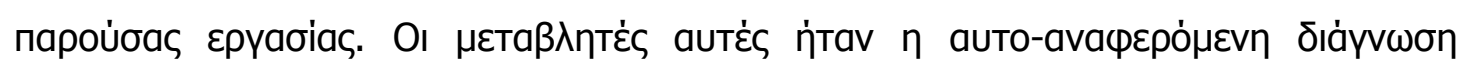

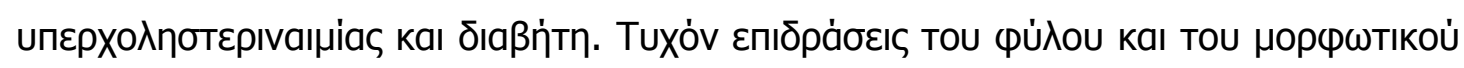

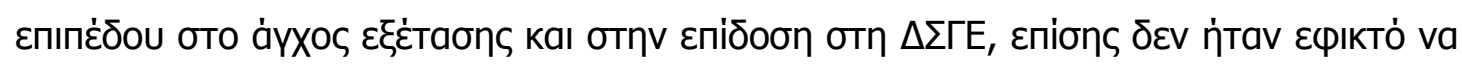

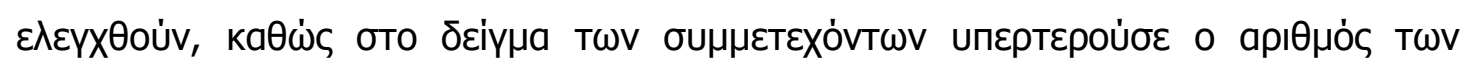

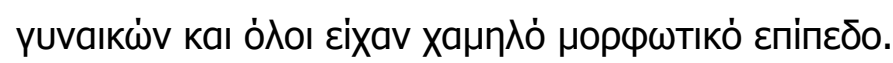

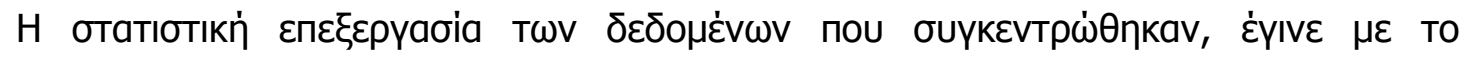

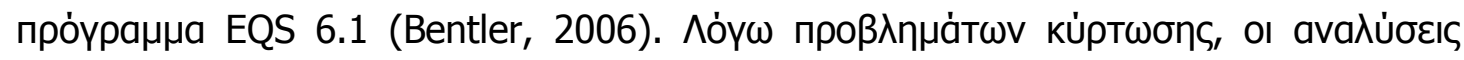

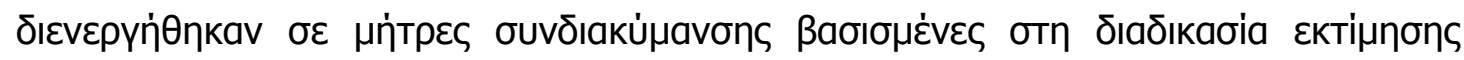

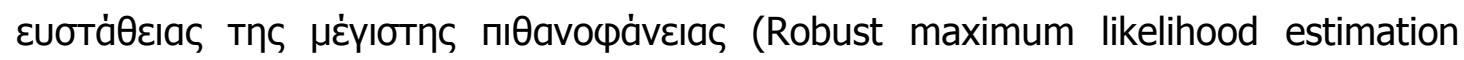
procedure. $\beta \lambda$. Bentler, 2006).

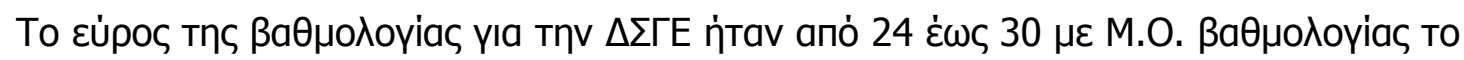

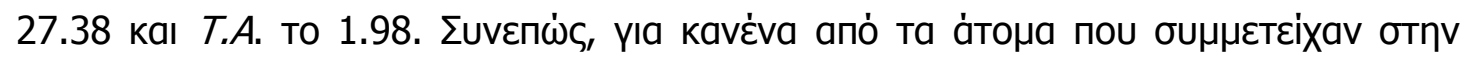

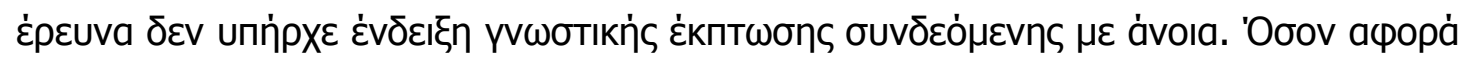

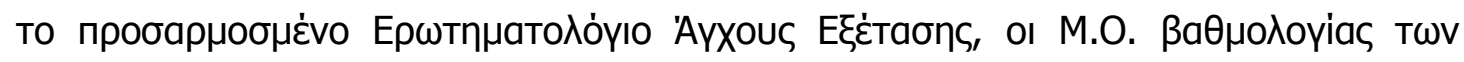

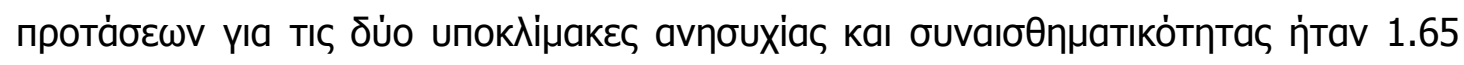

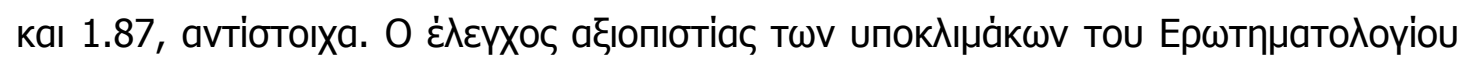

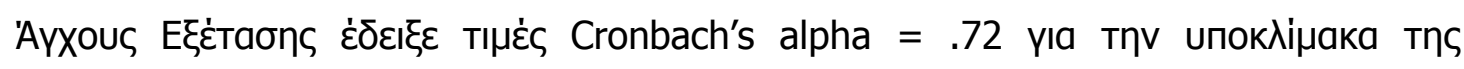

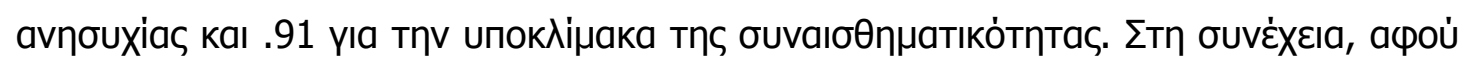

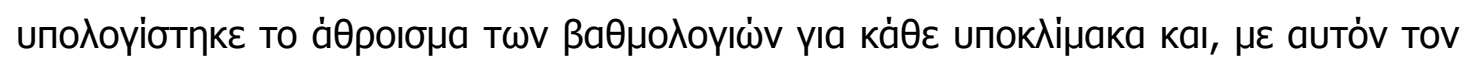




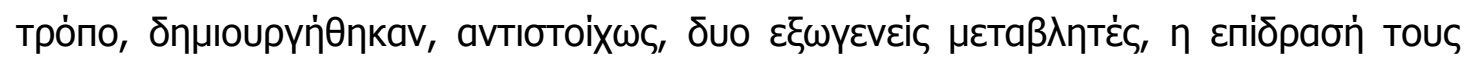

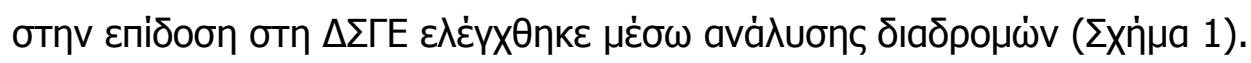

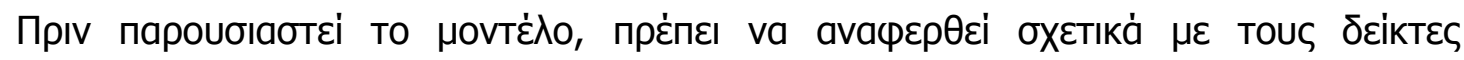

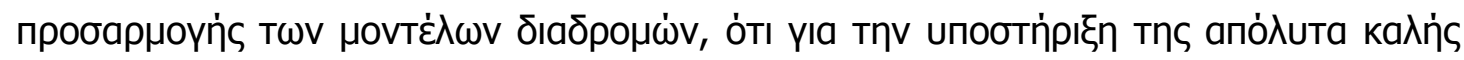

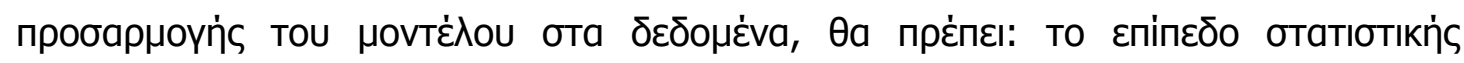

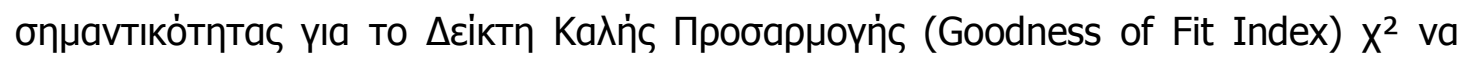

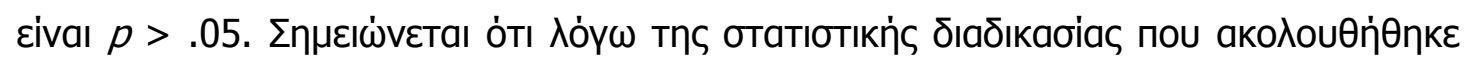

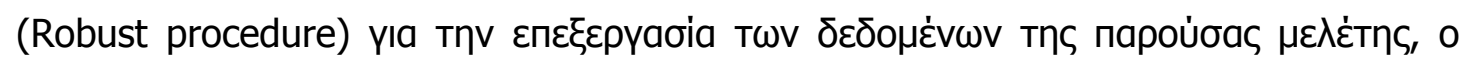

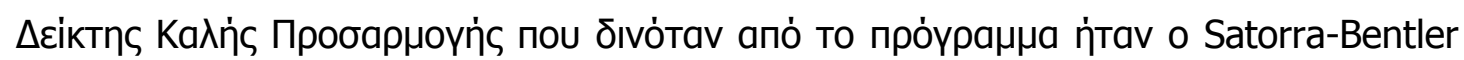

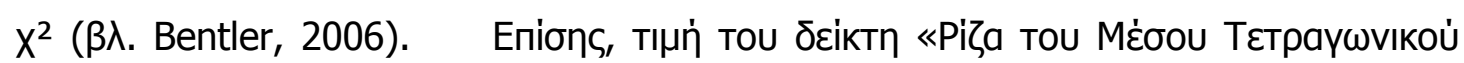

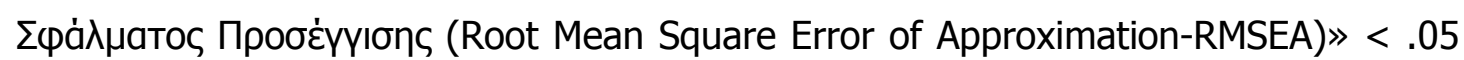

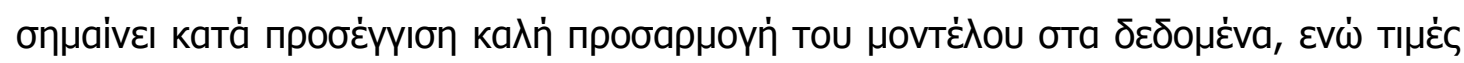

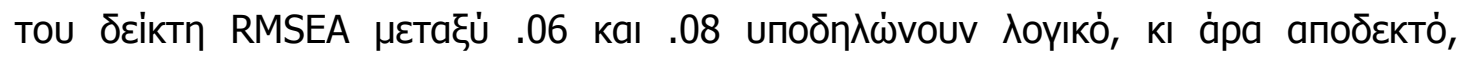

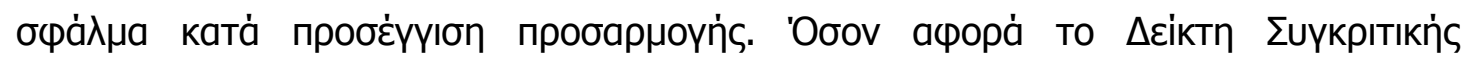

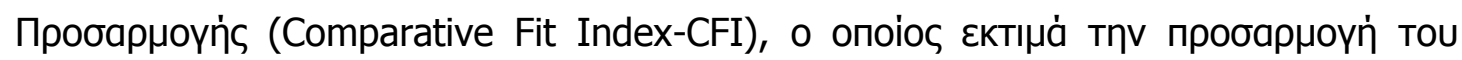

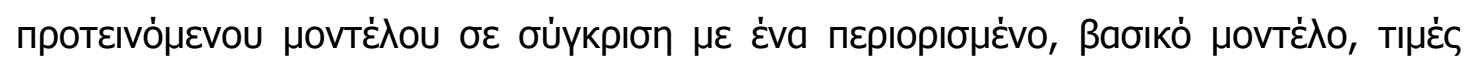

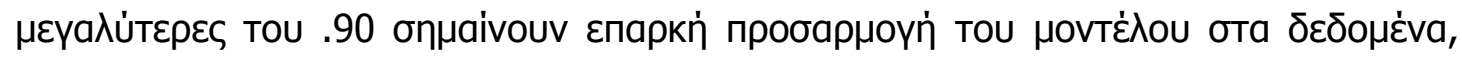

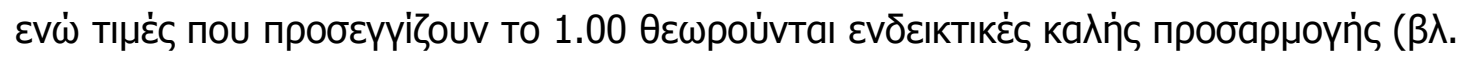

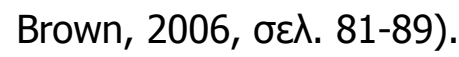

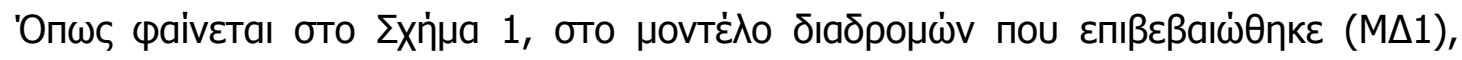
Satorra-Bentler $\chi^{2}(1,60)=.109, p>.05$, CFI $=1.00$, RMSEA $=.00$ (90\% CI: .00-

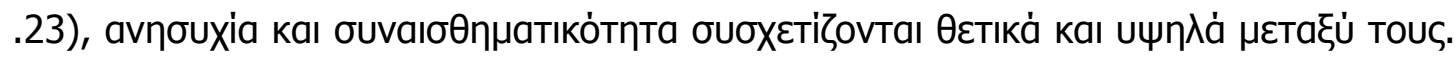

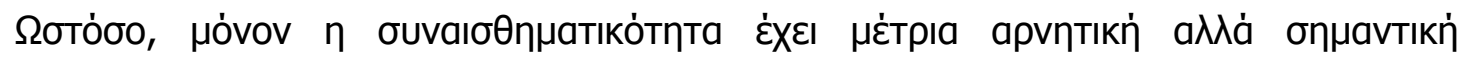

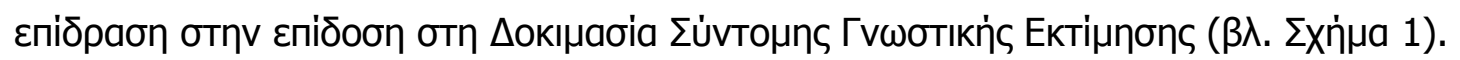




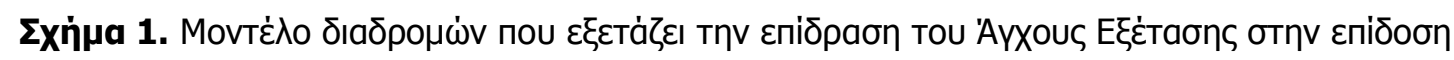

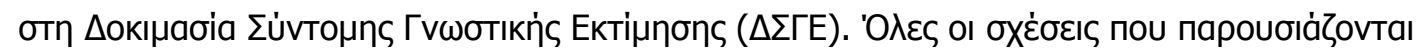

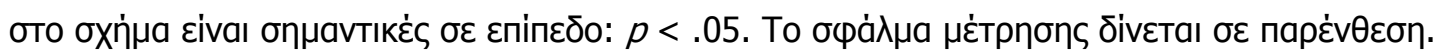

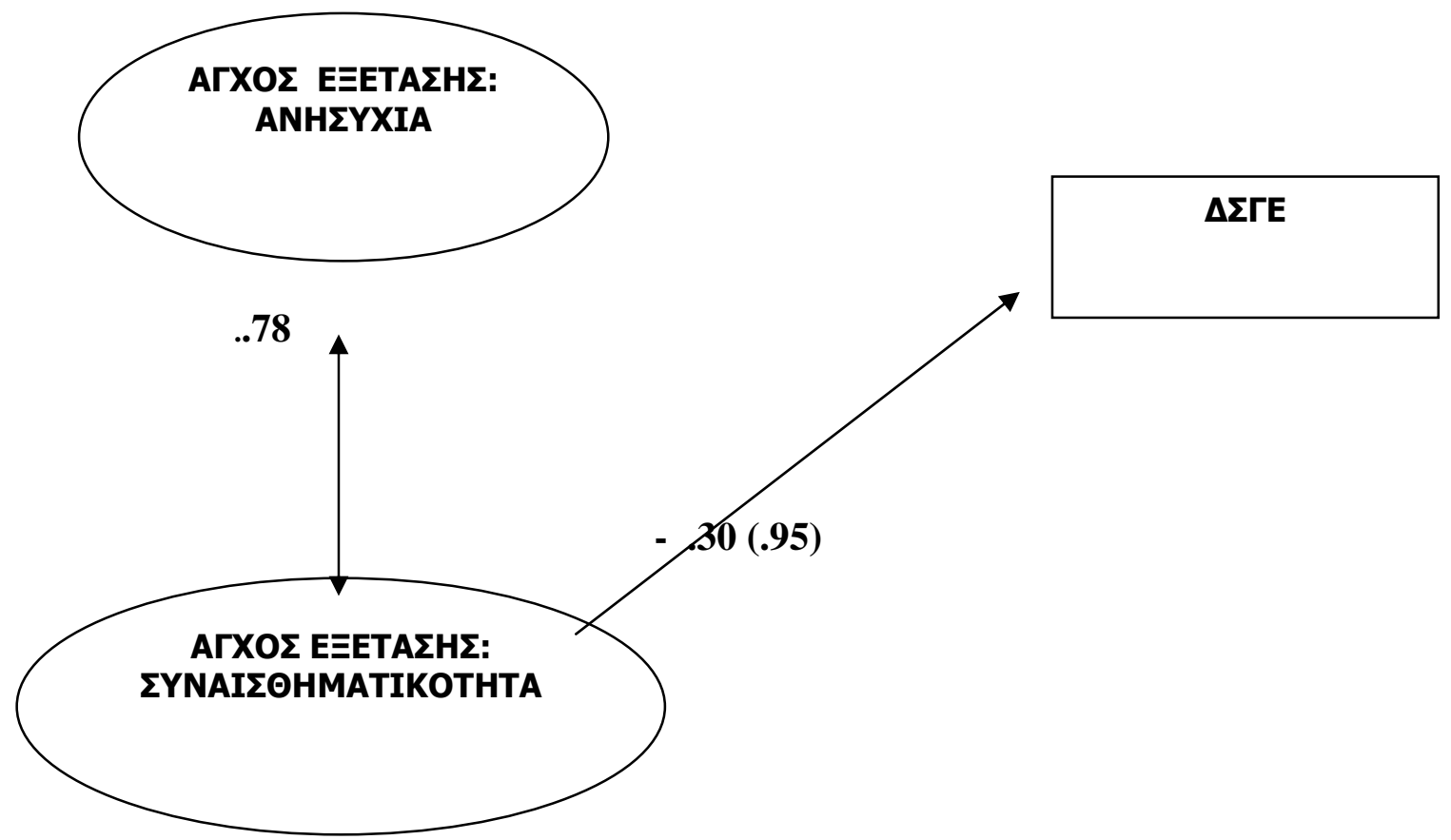

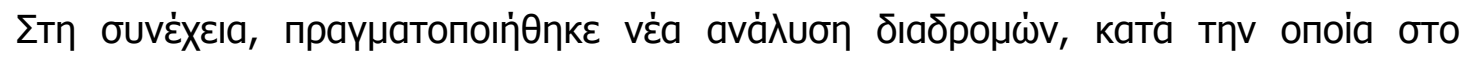

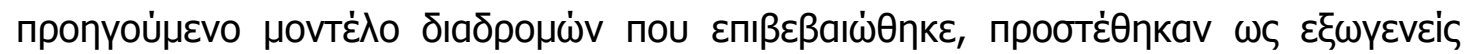

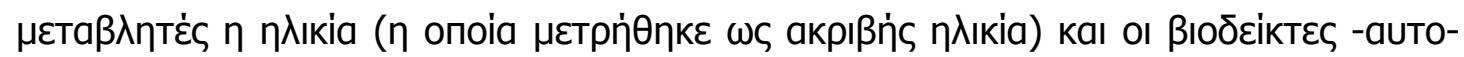

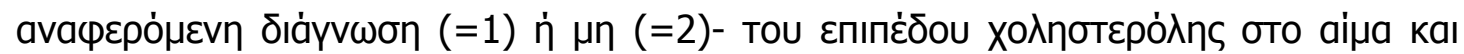

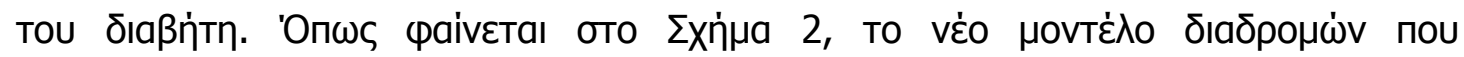
$\varepsilon п ґ \varepsilon \beta a ı \dot{\theta} \eta \kappa \varepsilon(M \Delta 2)$, Satorra-Bentler $X^{2}(4,60)=2.700, p>.05$, CFI = 1.00,

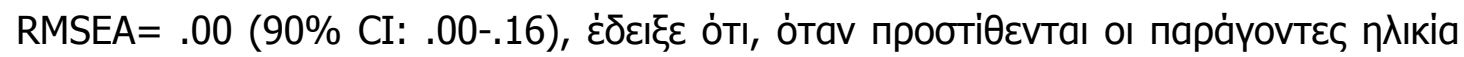

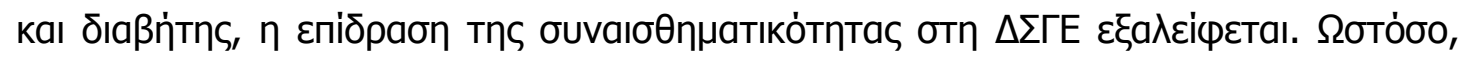

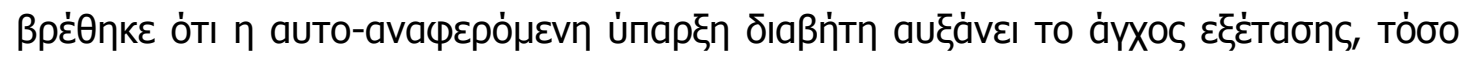

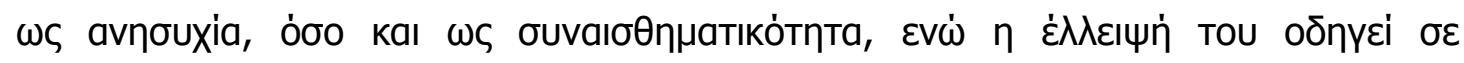

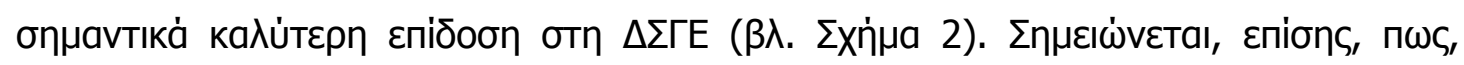

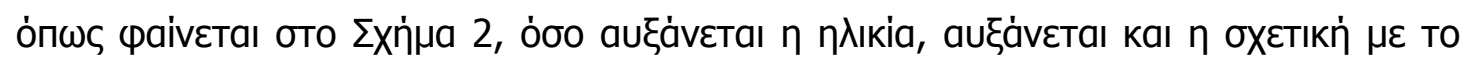

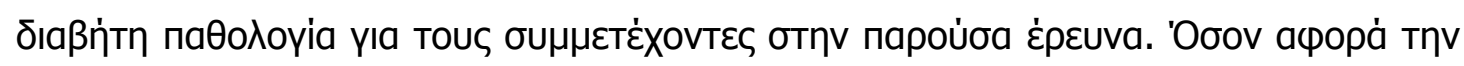

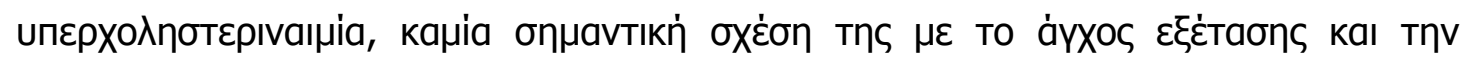

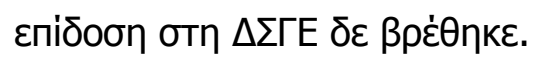




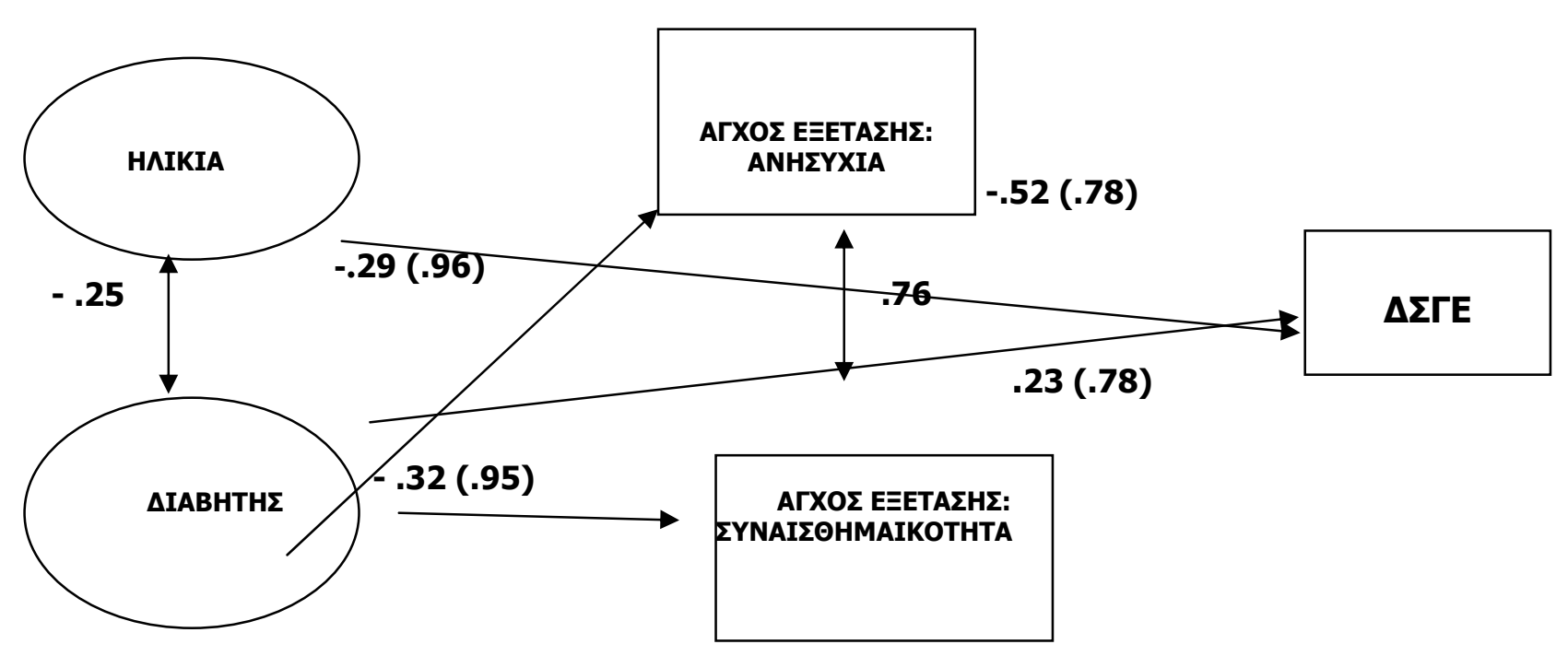

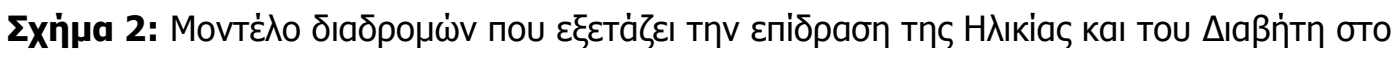

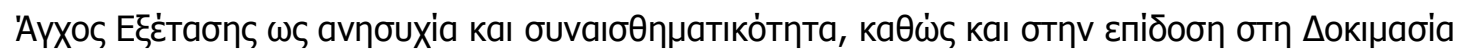

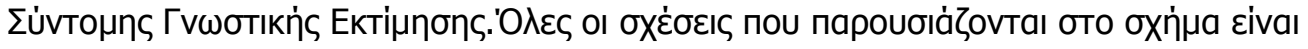

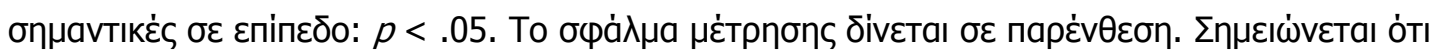

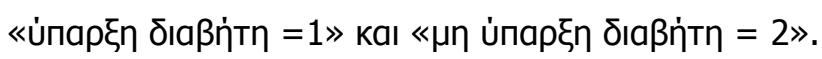

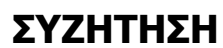

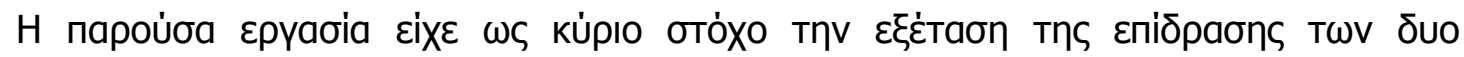

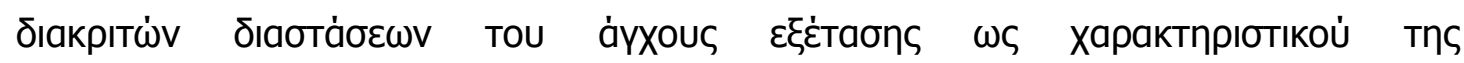

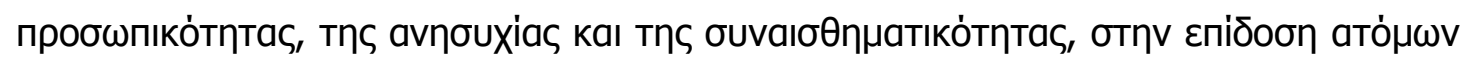

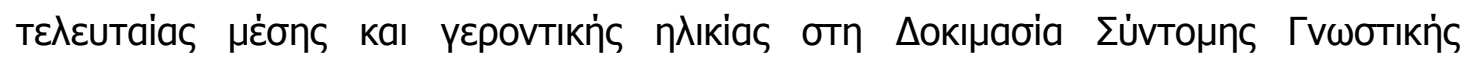

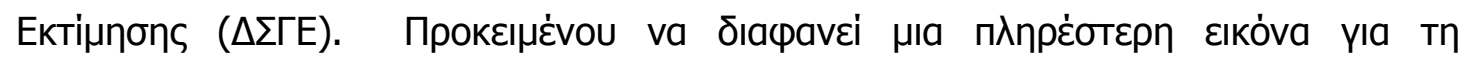

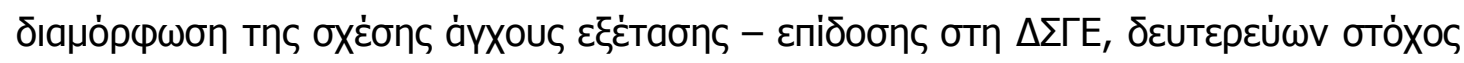

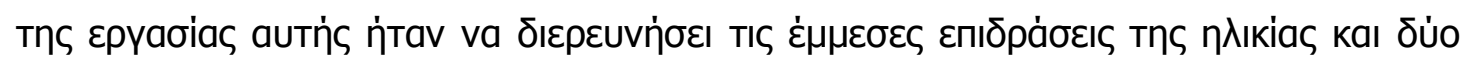

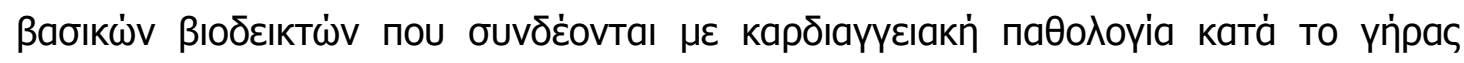

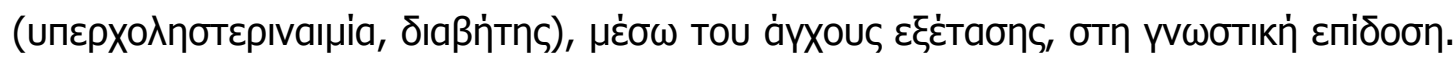

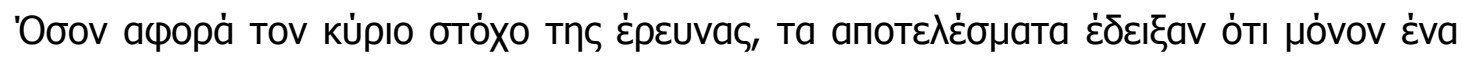

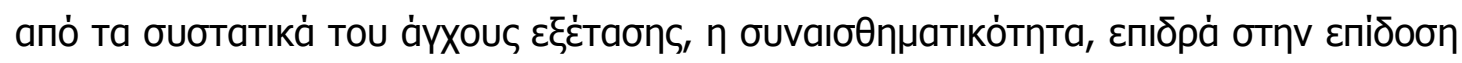

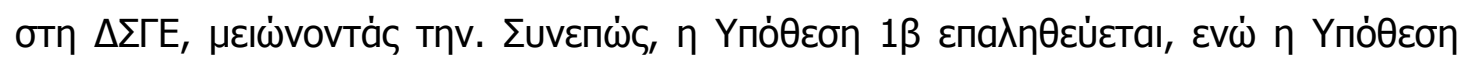

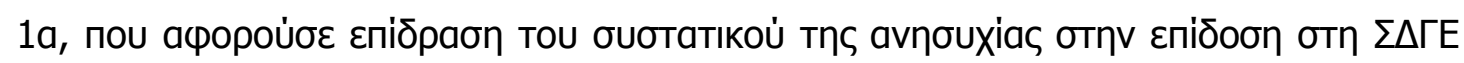

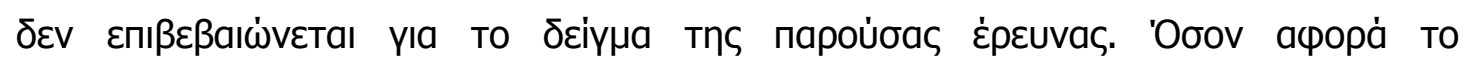

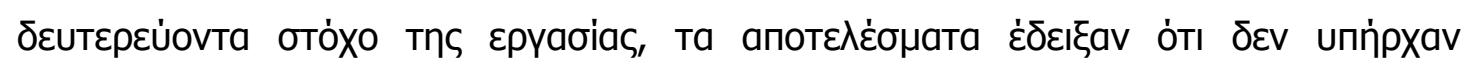

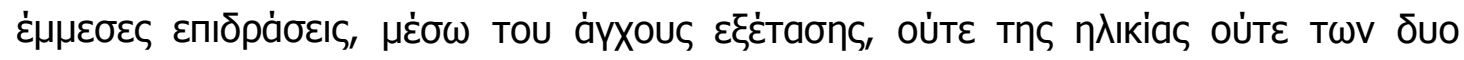




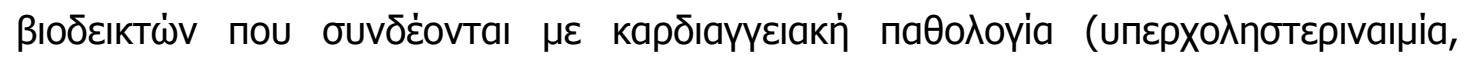

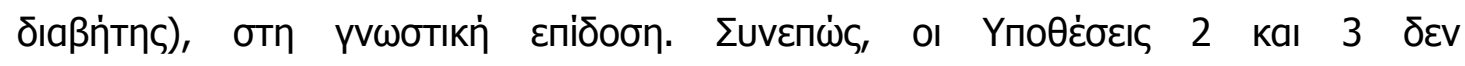

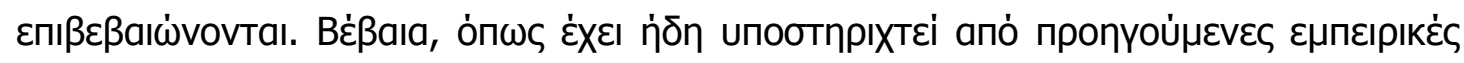

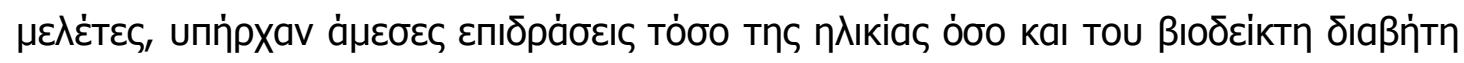

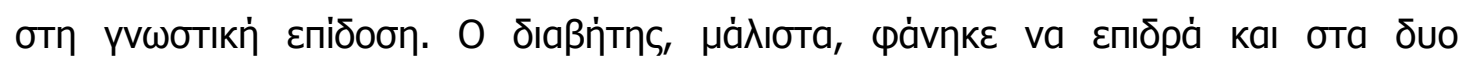

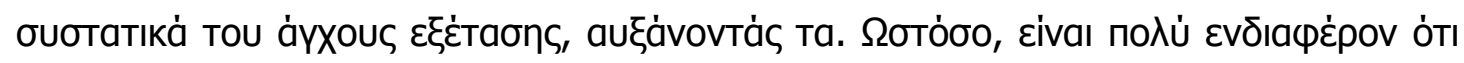

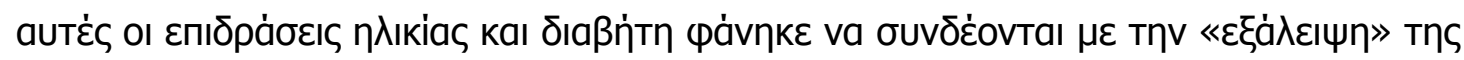

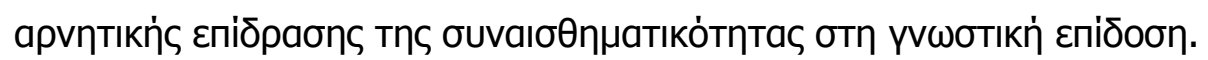

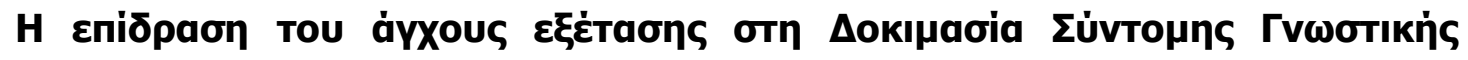 Eктijnons}

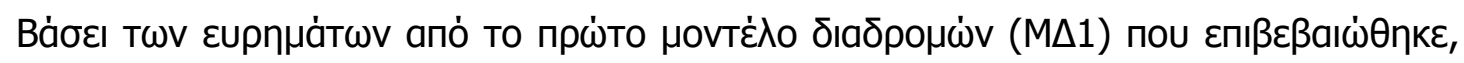

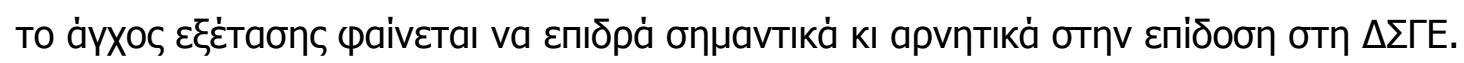

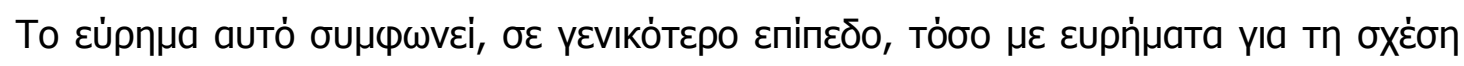

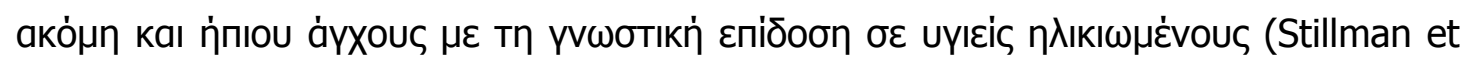

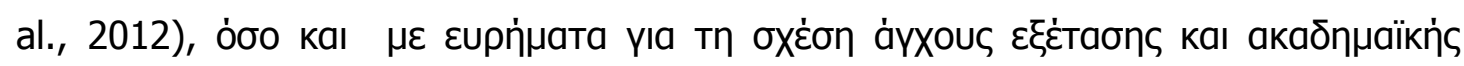

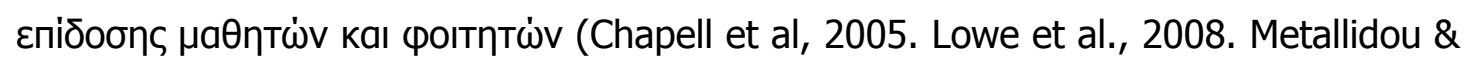

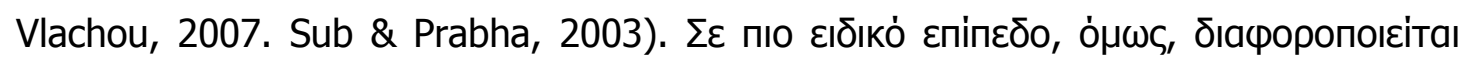

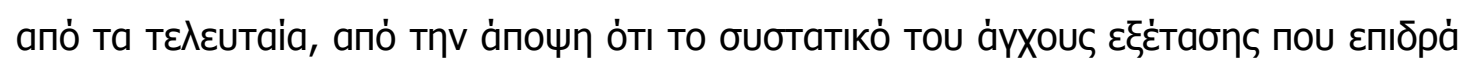

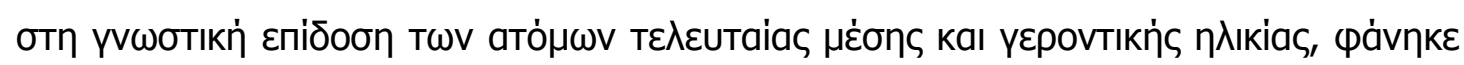

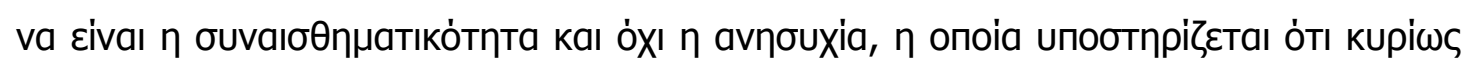

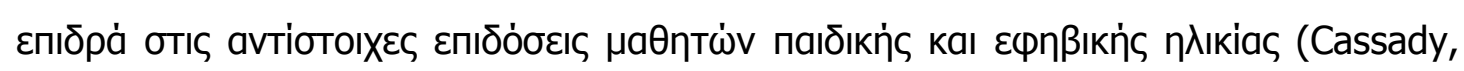

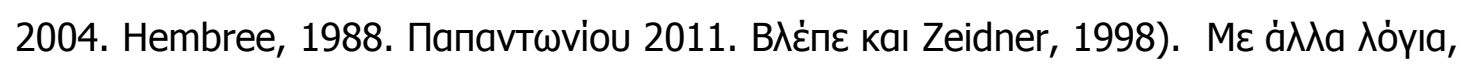

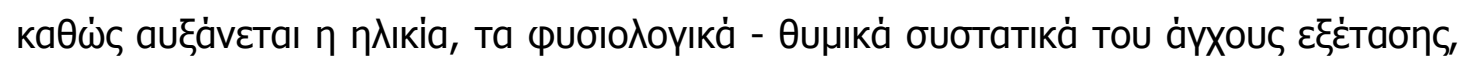

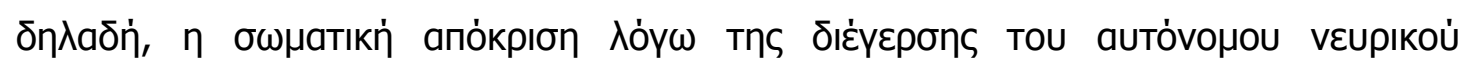

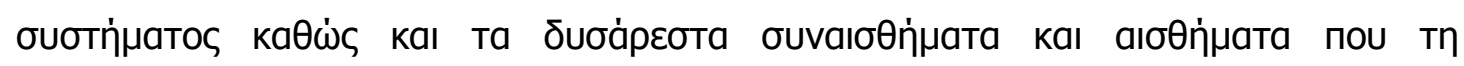

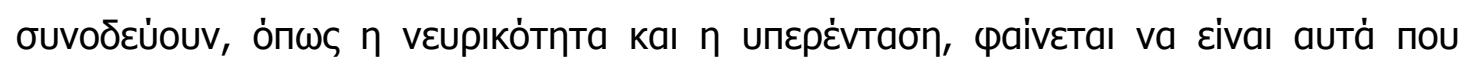

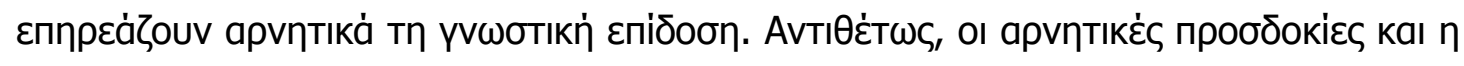

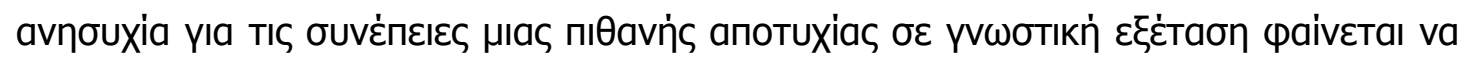

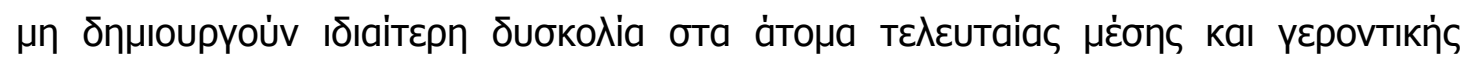

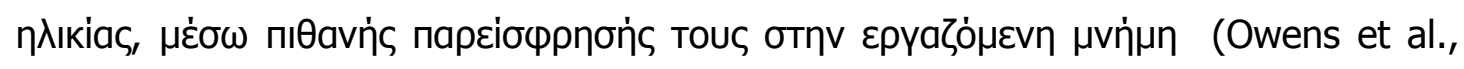

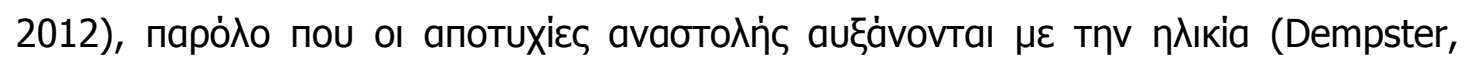

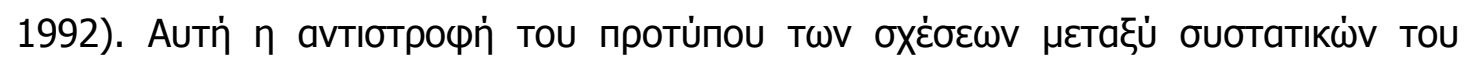

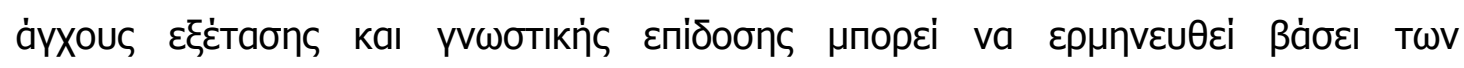




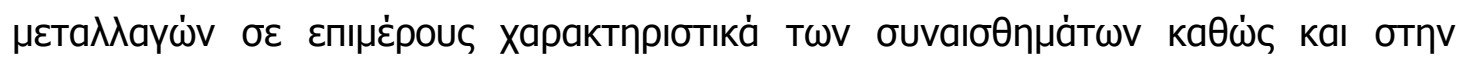

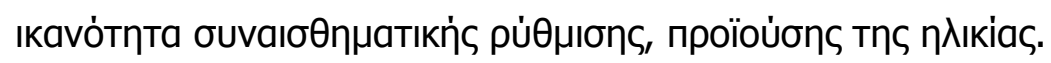

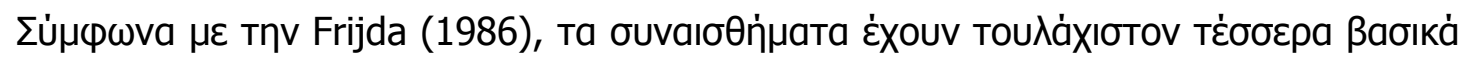

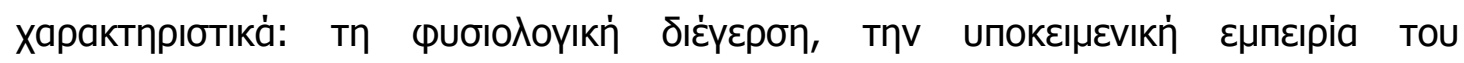

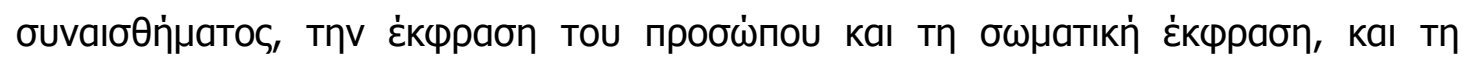

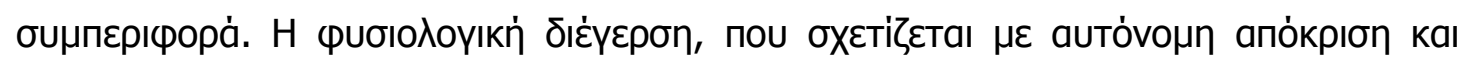

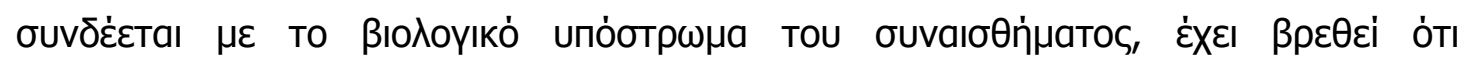

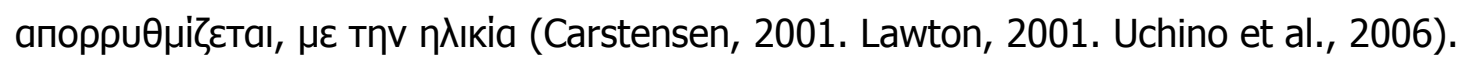

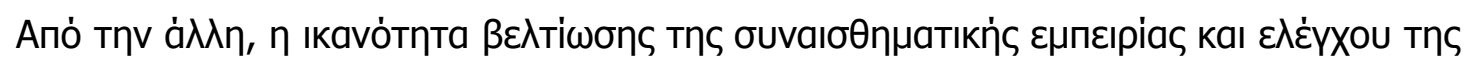

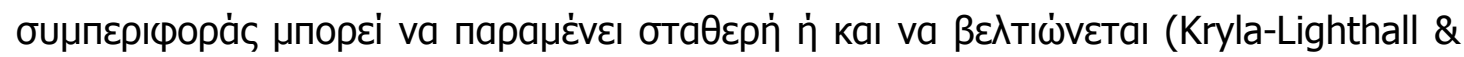

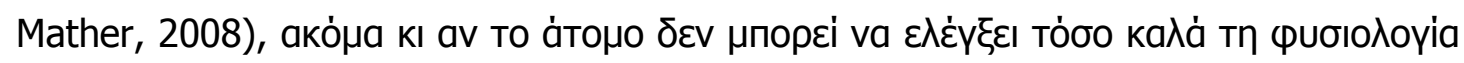

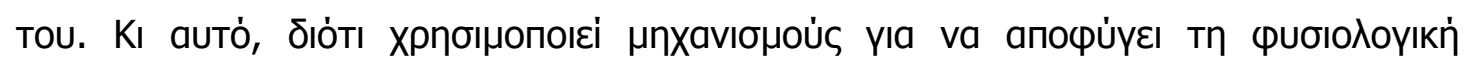

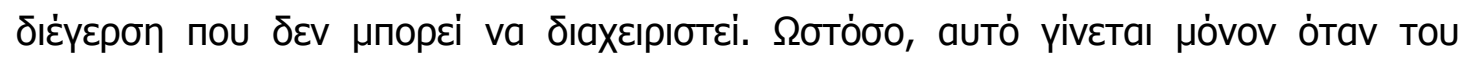

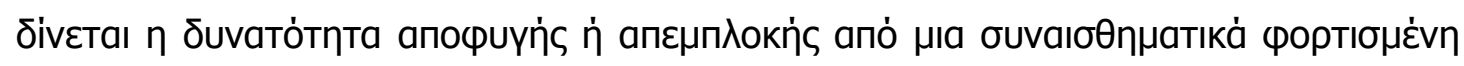

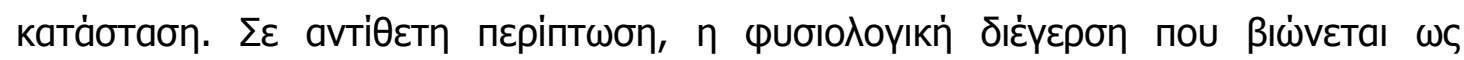

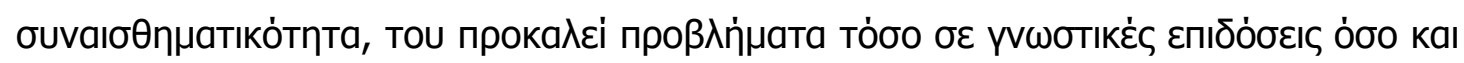

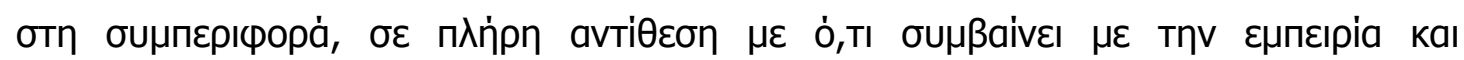

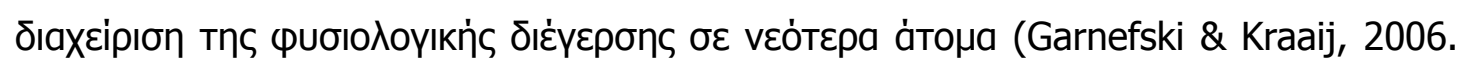
Kryla- Lighthall \& Mather, 2008. Thomsen, Mehlsen, Viidik, Sommerlund \& Zachariae, 2005).

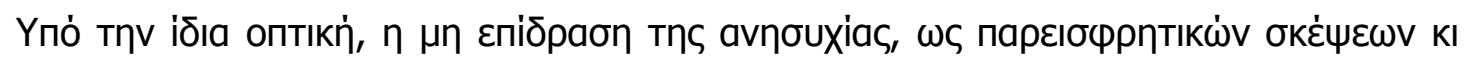

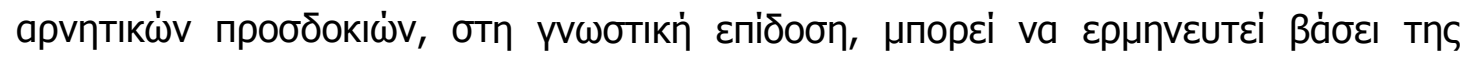

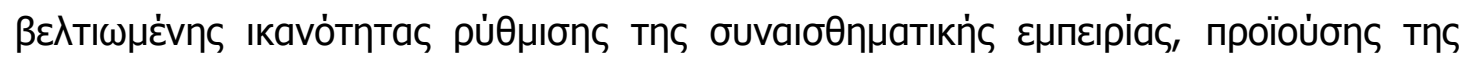

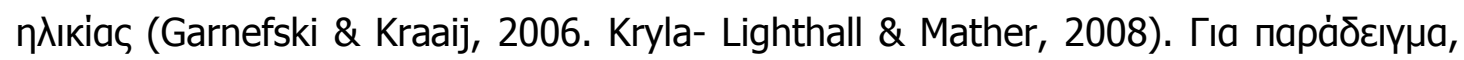

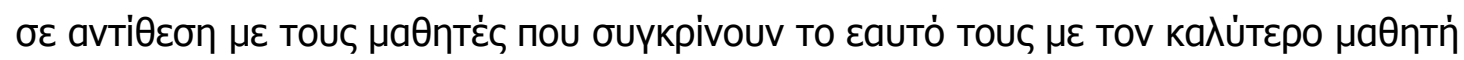

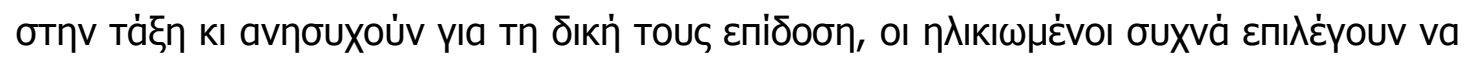

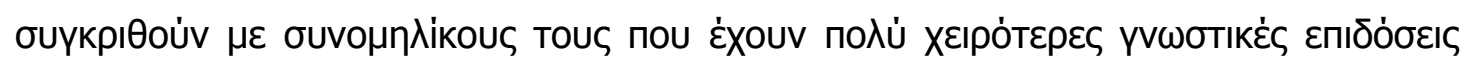

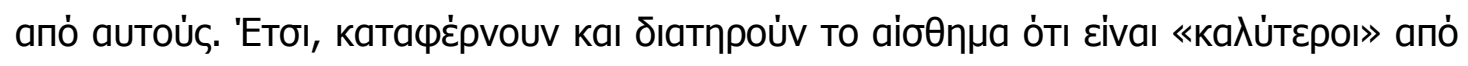

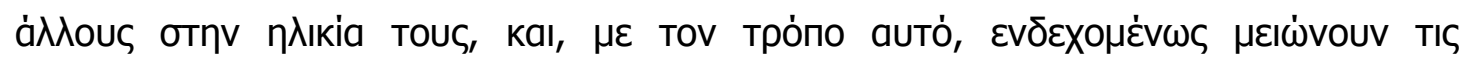

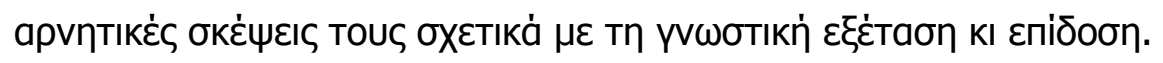




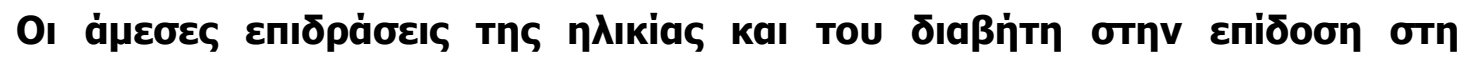

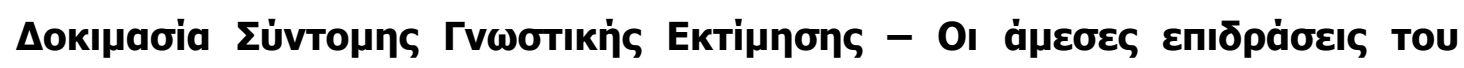

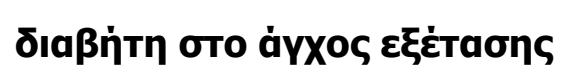

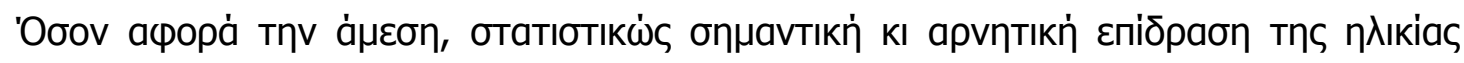

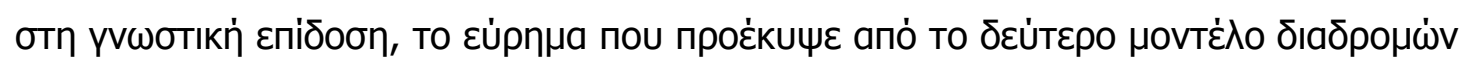

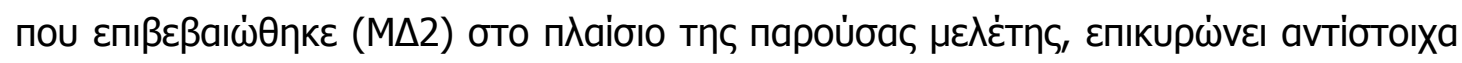

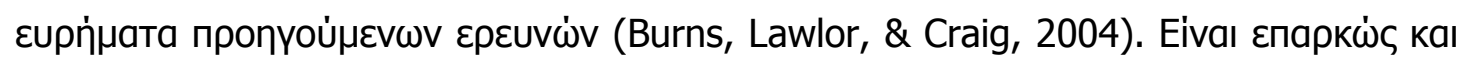

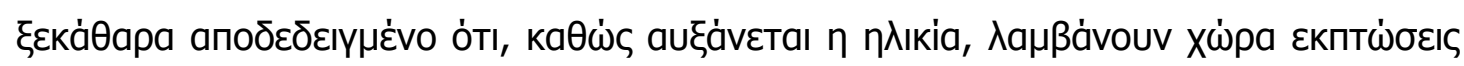

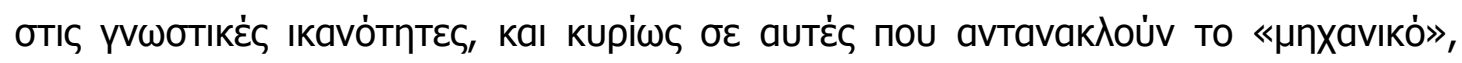

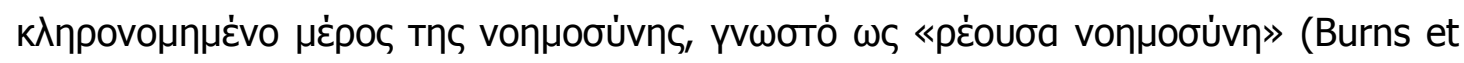

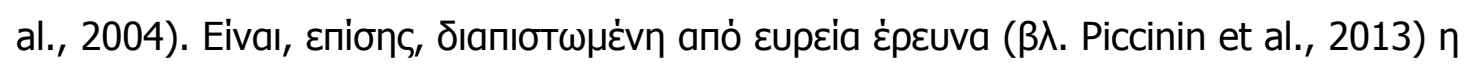

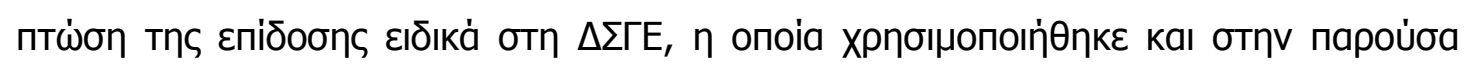

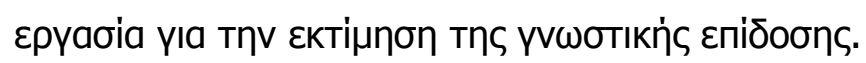

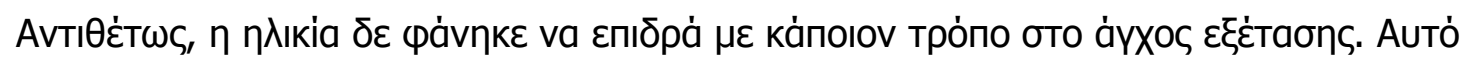

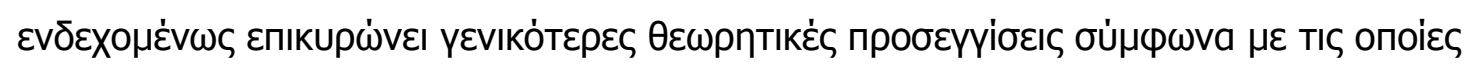

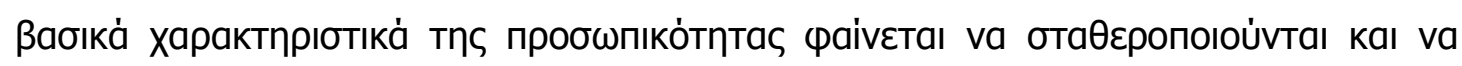

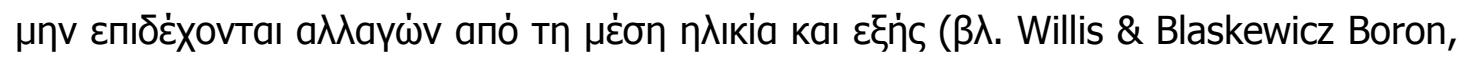
2008).

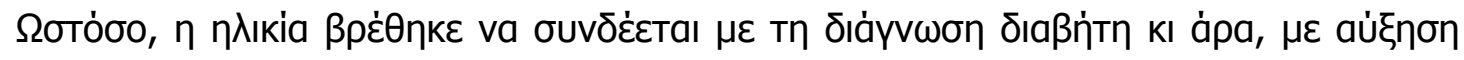

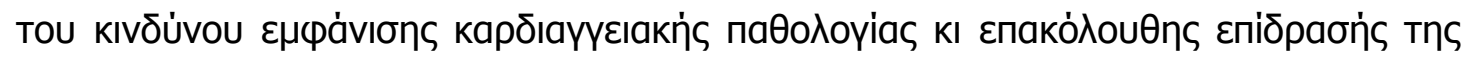

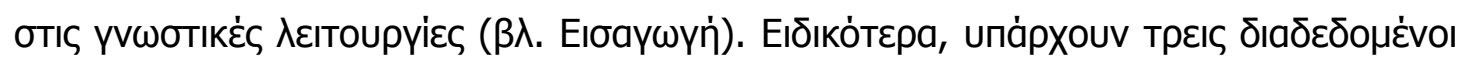

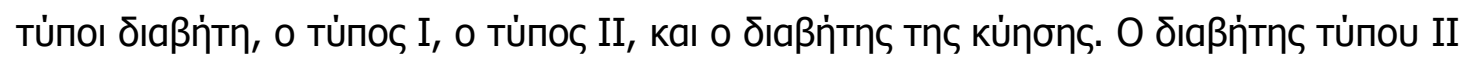

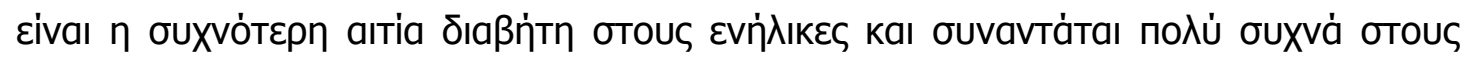

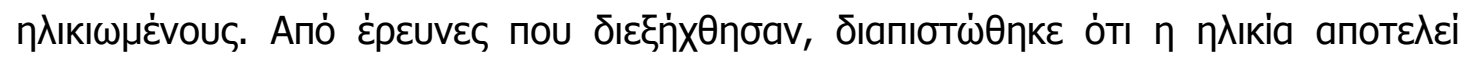

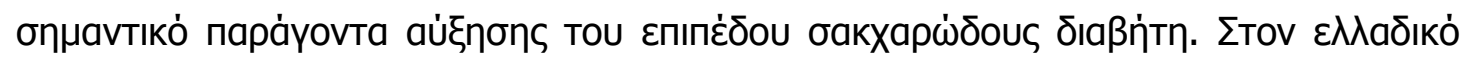

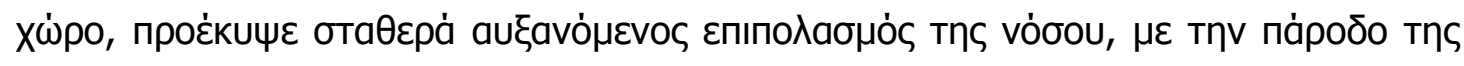

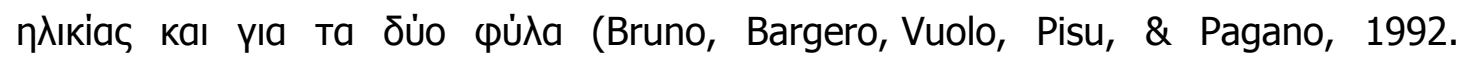

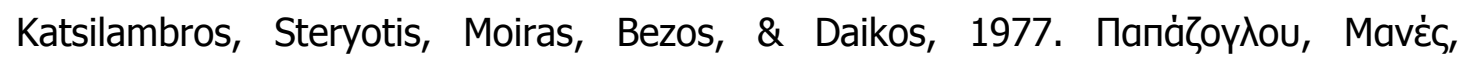

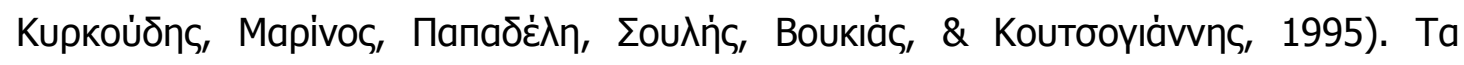

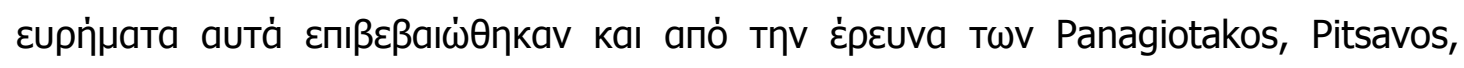

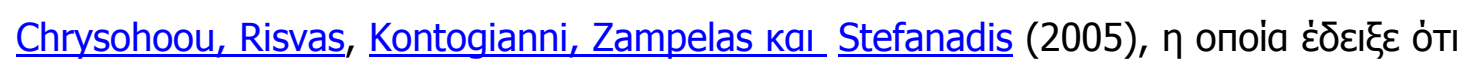

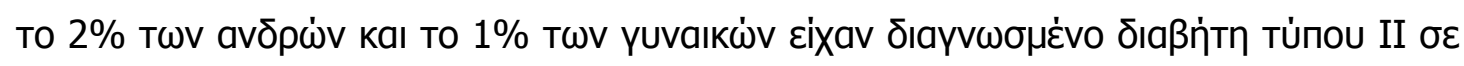




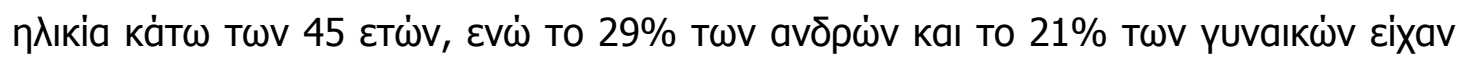

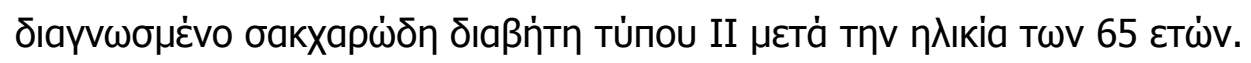

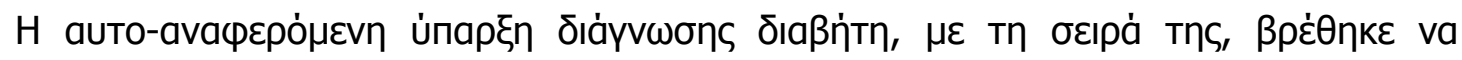

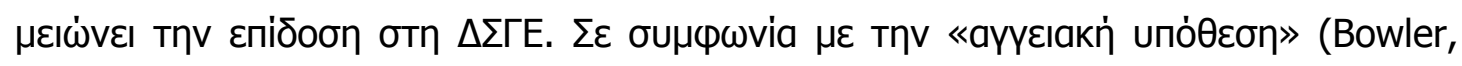

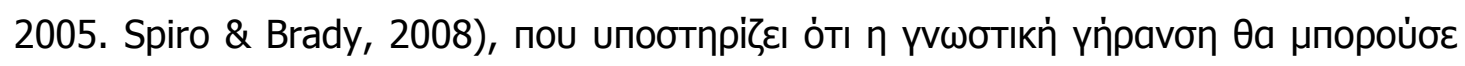

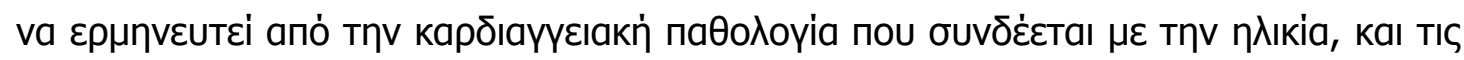

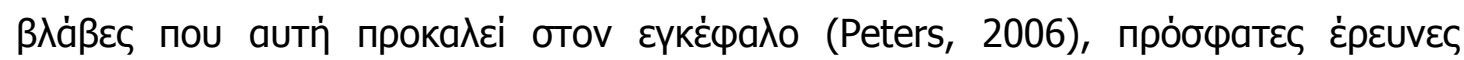

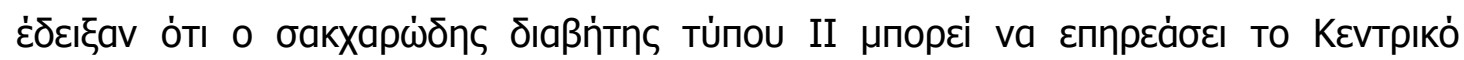

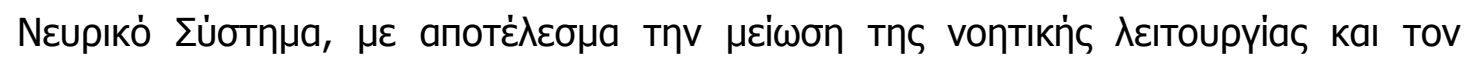

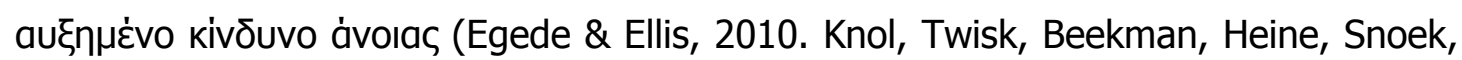

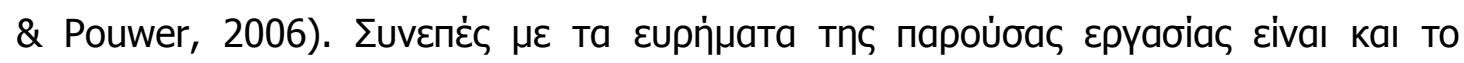

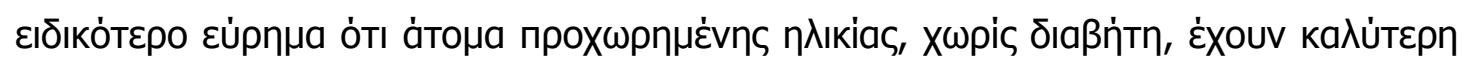

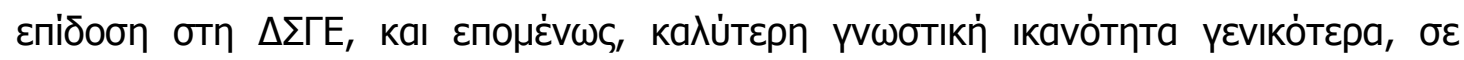

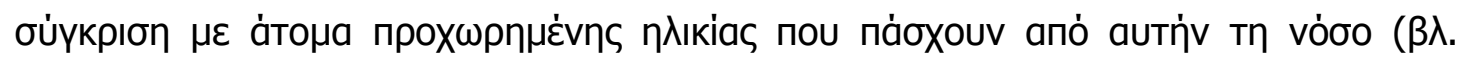

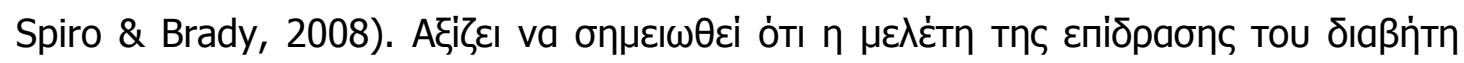

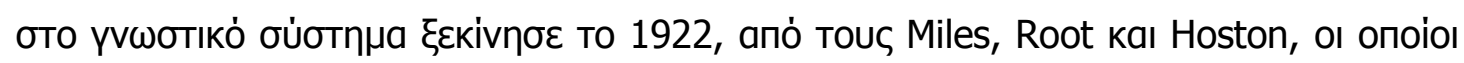

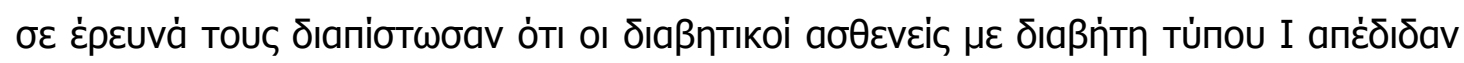

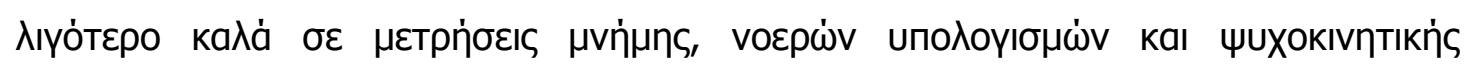

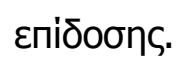

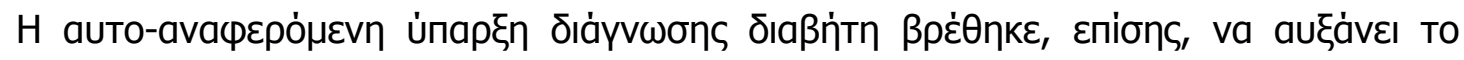

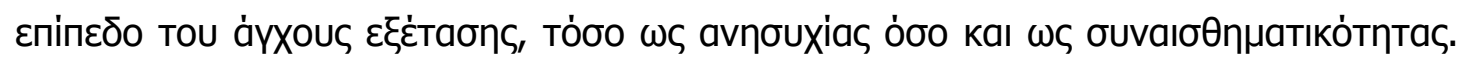

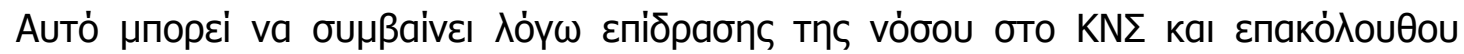

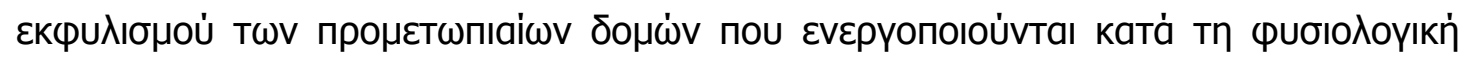

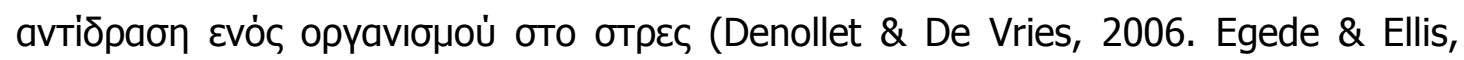

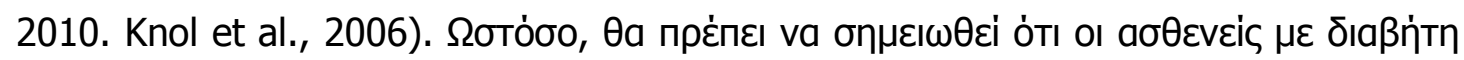

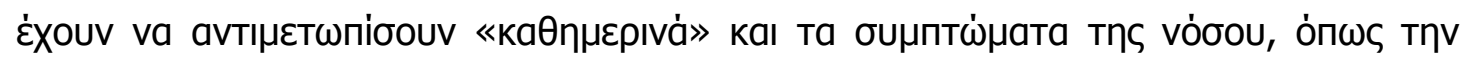

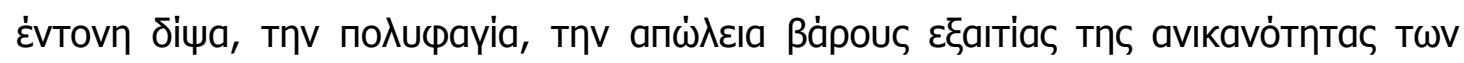

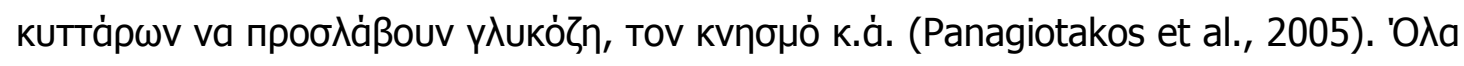

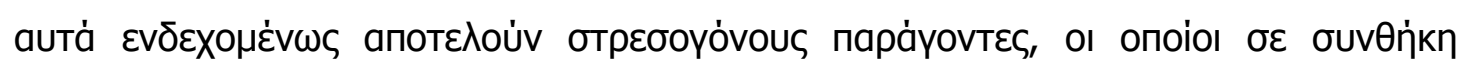

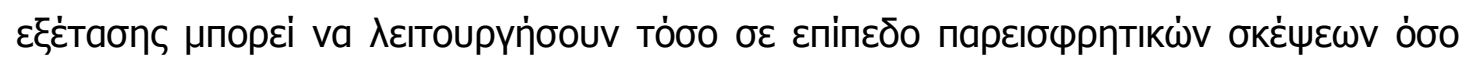

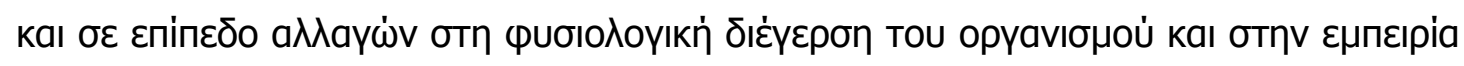

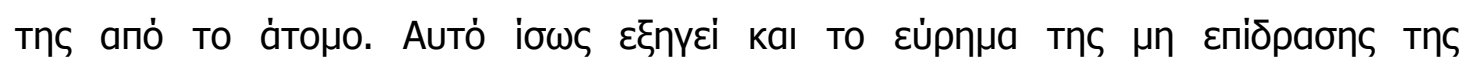

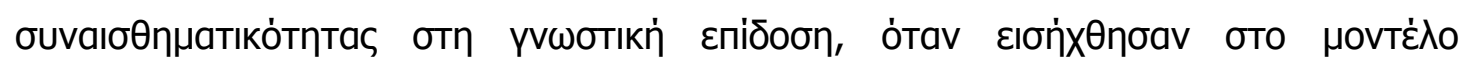




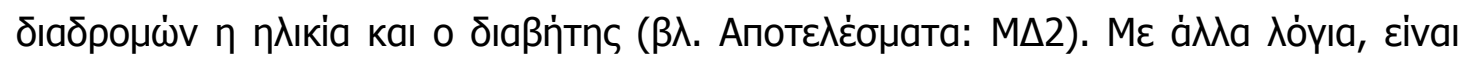

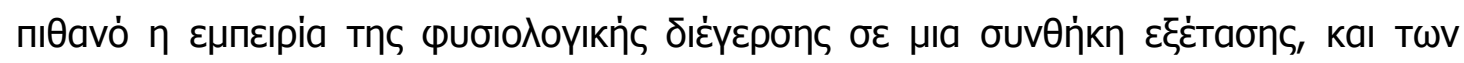

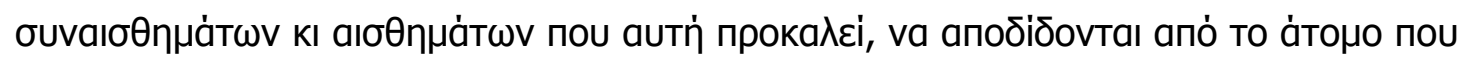

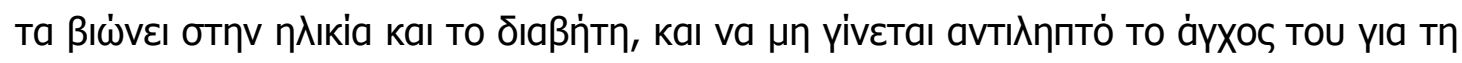

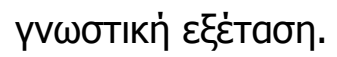

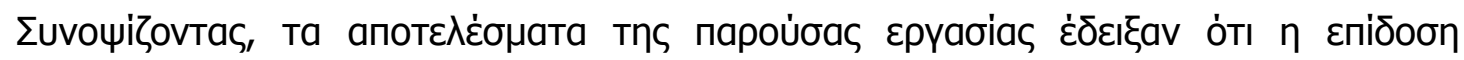

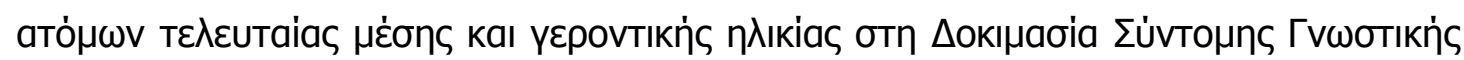

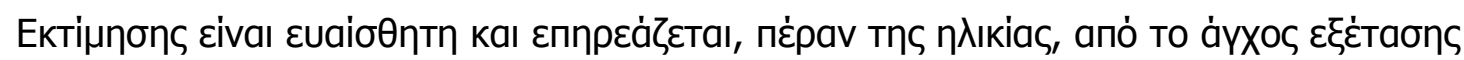

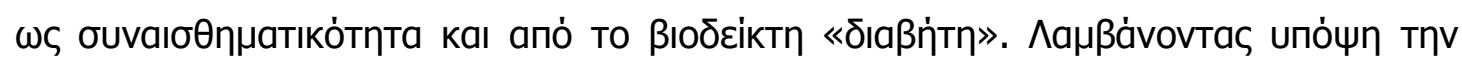

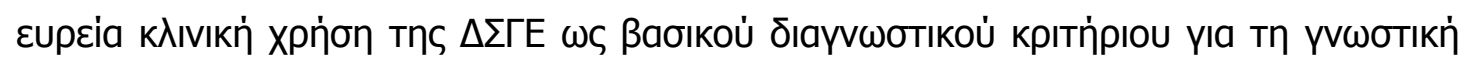

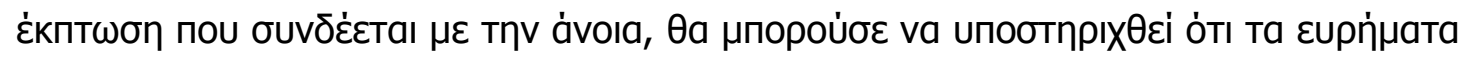

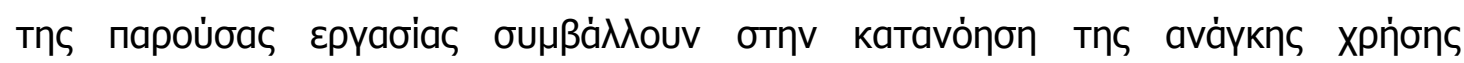

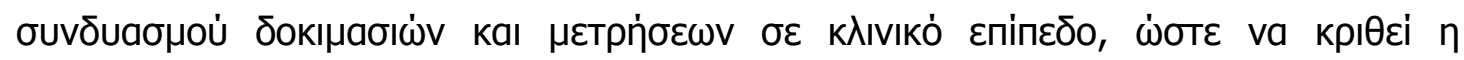

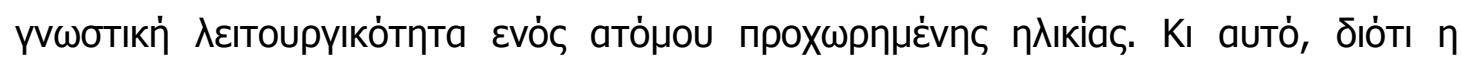

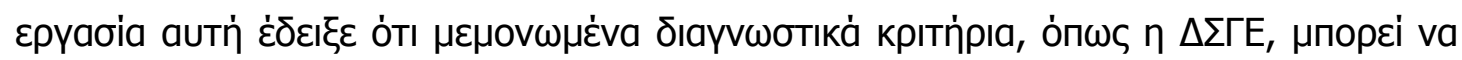

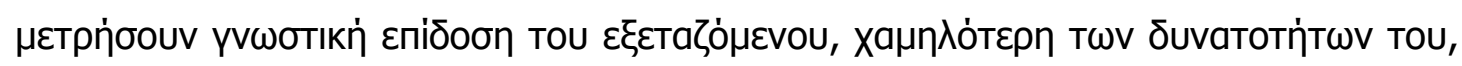

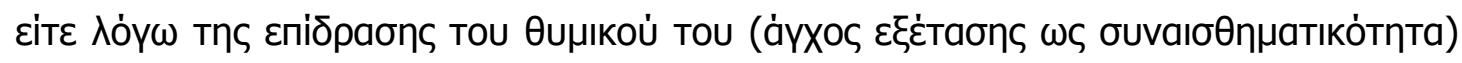

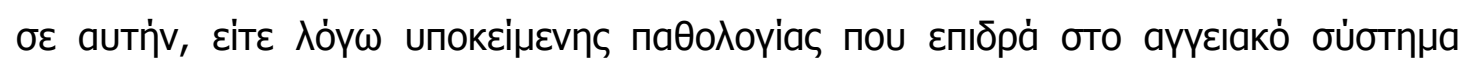
(п.Х., ठıаßंтпһ).

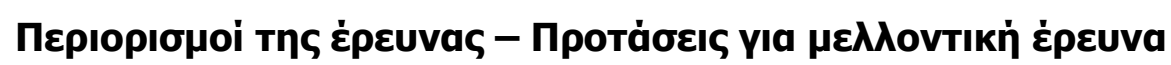

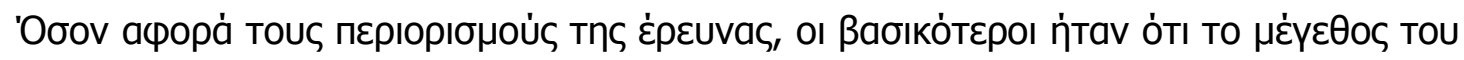

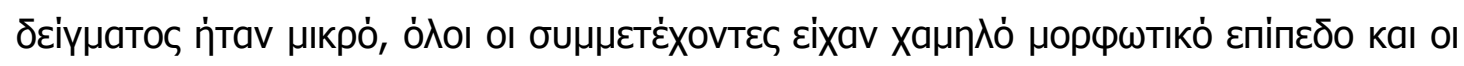

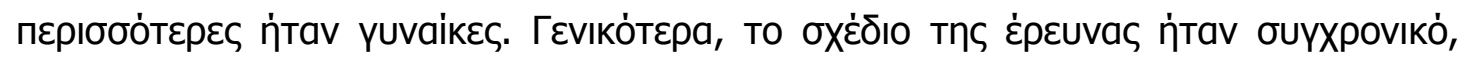

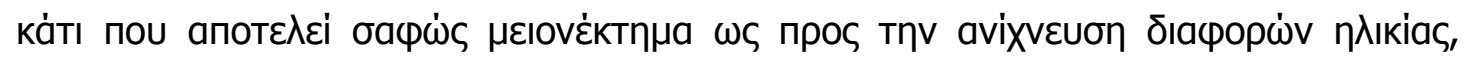

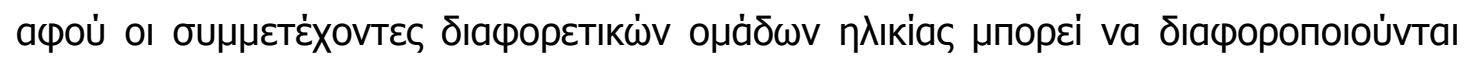

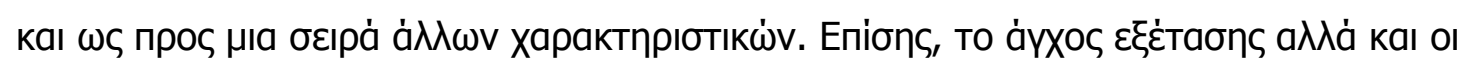

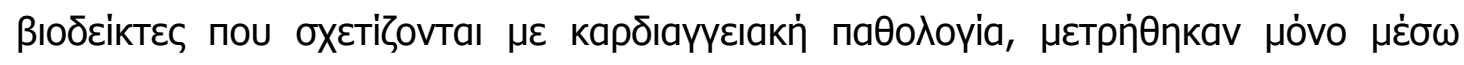

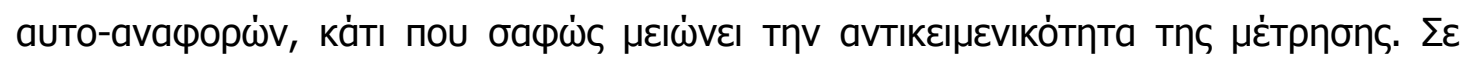

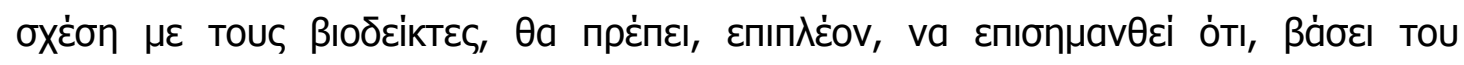

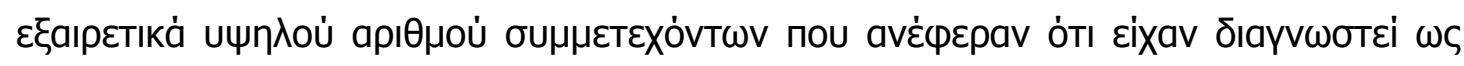

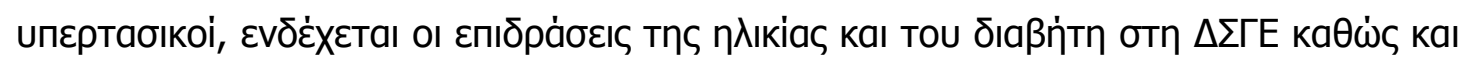

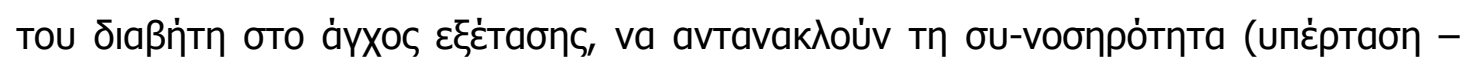

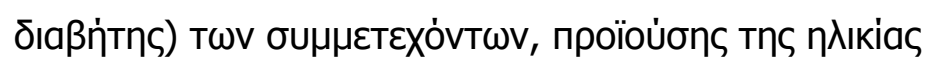




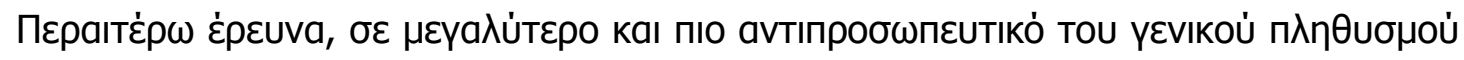

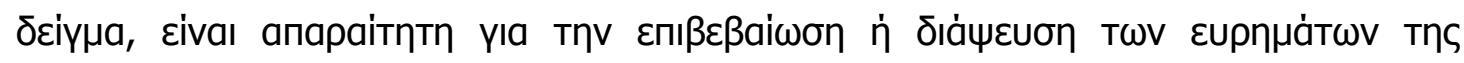

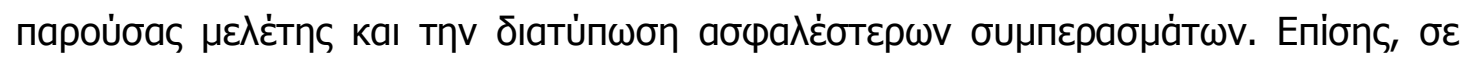

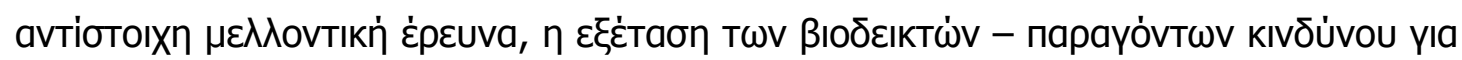

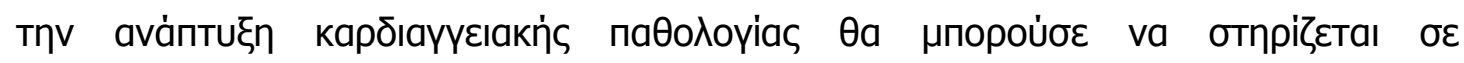

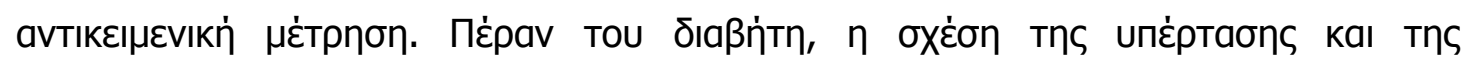

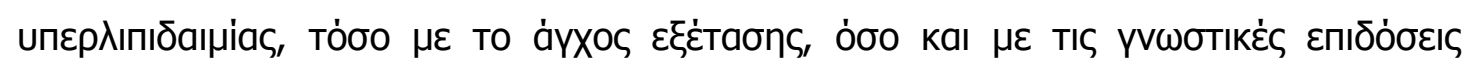

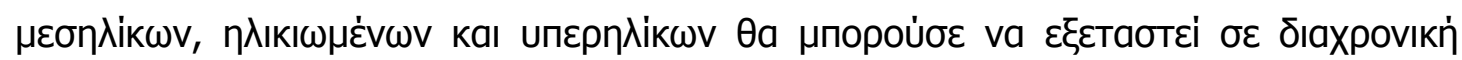

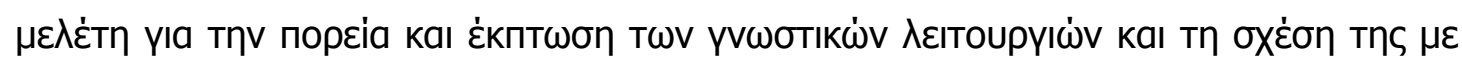

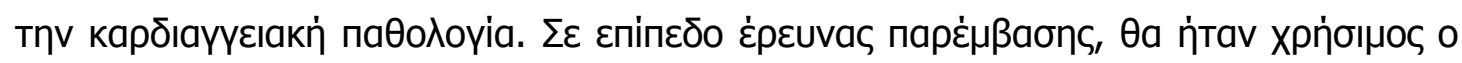

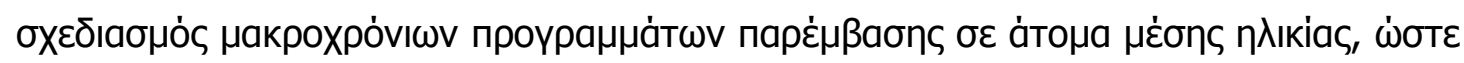

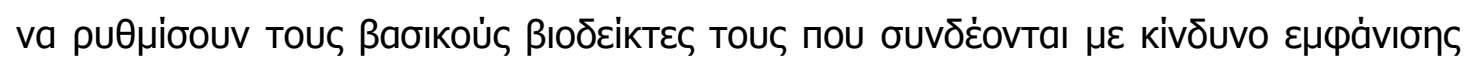

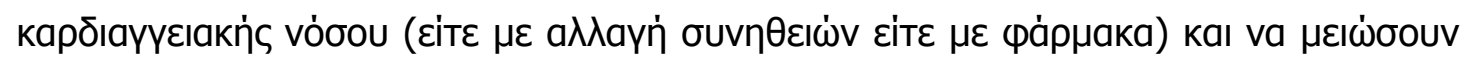

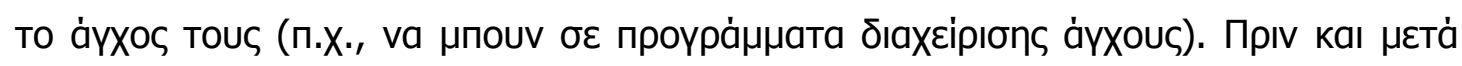

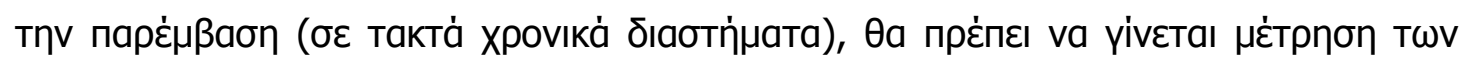

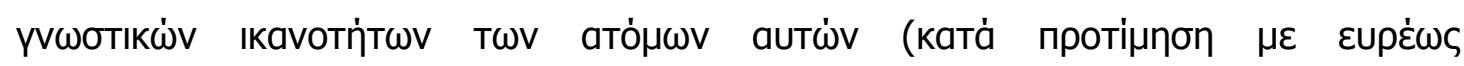

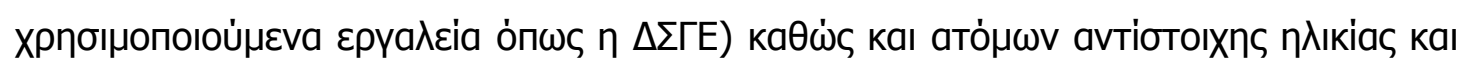

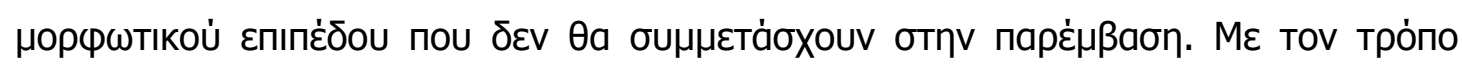

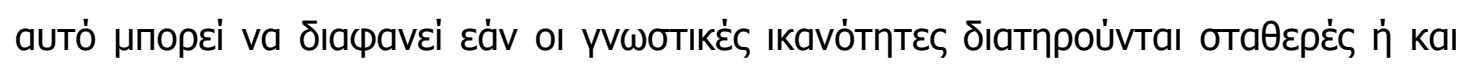

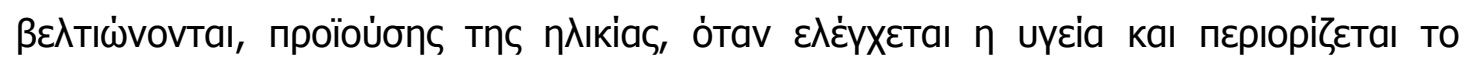

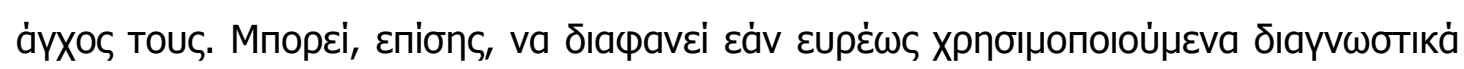

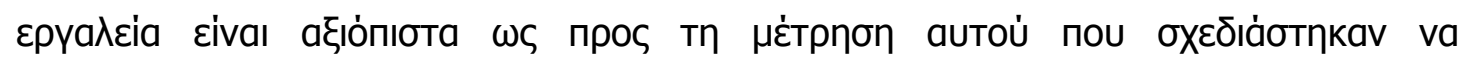

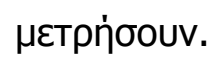




\section{ВIВАIОГРАФІА}

Albert, M. \& Cohen, C. (1992). The test for severe impairment: An instrument for the assessment of patients with severe cognitive dysfunction. Journal of American Geriatrics Society, 40(5), 449-453.

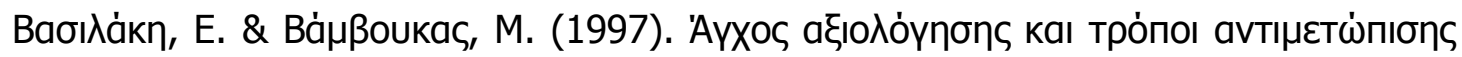

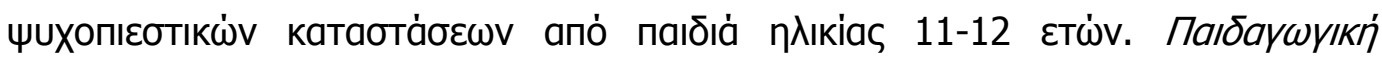

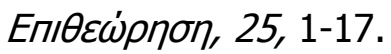

Bengtson, V., Gans, D., Putney, N., \& Silverstein, M. (Eds.) (2009). Handbook of theories of aging, (2nd Edition, pp. 323-344). New York: Springer.

Bentler, P. (2006). EQS 6 Structural equations program manual. Encino, CA: Multivariate Software, Inc.

Berlyne, D. E. (1960). Conflict, arousal, and curiosity. New York: McGraw-Hill.

Berlyne, D. E. (1963). Motivational problems raised by exploratory and epistemic behavior. In S. Koch (Ed.), Psychology: A study of a science (Vol. 5, pp. 284364). New York: McGraw-Hill.

Bowler, J. V. (2005). Vascular cognitive impairment. Journal of Neurology, Neurosurgery, and Psychiatry, 76, 35-44.

Brown, T. A. (2006). Confirmatory factor analysis for applied research. New York: The Guilford Press.

Bruno, G., Bargero, G., Vuolo, A., Pisu, E., \& Pagano, G. (1992). A population-based prevalence survey of known diabetes mellitus in Northern Italy based upon multiple independent sources of ascertainment. Diabetologia, 35(9), 851-856.

Burns, A., Lawlor, B., \& Craig, S. (2004). Assessment scale in old age psychiatry. New York: Martin Dunitz.

Carstensen, L. (2001). Emotion and aging. In G. Maddox, (Eds.), Encyclopedia of Aging. (3rd ed., pp. 327-329). New York: Springer.

Cassady, J. C. (2004). The impact of cognitive test anxiety on text comprehension and recall in the absence of external evaluative pressure. Applied Cognitive Psychology, 18, 311-325.

Cauley, J., Dorman, J., \& Ganguli, M. (1997). Genetic and aging epidemiology. The merging of two disciplines. Neurologic Clinics, 14(2), 467-475.

Chapell, M. S., Blanding, Z. B., Silverstein, M. E., Takahashi, M., Newman, B., Gubi, A., \& McCann, N. (2005). Test anxiety and academic performance in undergraduate and graduate students. Journal of Educational Psychology, 97, 268-274. 
Conley, K. \& Lehman, B. (2012). Test anxiety and cardiovascular responses to daily academic stressors. Stress and Health, 28, 41-50.

Dempster, F. (1992). The rise and fall of the inhibitory mechanism: Toward a unified theory, M., Folstein, S., \& McHugh, P. (1975). "Mini-Mental State": A practical method for grading the cognitive state of patients for the clinician. Journal of Psychiatric Research, 12, 189-198.

Foreman, M. (1987). Reliability and validity of mental status questionnaires in elderly hospitalized patients. Nursing Research, 36(4), 216-220.

Frijda, H. (1986). The emotions. UK: Cambridge University Press.

Garnefski, N. \& Kraaij, V. (2006). Relationships between cognitive emotion regulation strategies and depressive symptoms: A comparative study of five specific samples. Personality and Individual Differences, 40, 1659-1669.

Hebb, D. O. (1949). The organization of behavior. New York: Wiley.

Hebb, D. O. (1966). A textbook of psychology (2nd ed.). Philadelphia: Saunders.

Hembree, R. (1988). Correlates, causes, effects and treatment of test anxiety. Review of Educational Research, 58, 47-77.

Hofer, S. \& Alwin, D. (Eds.) (2008). Handbook of cognitive aging: interdisciplinary perspectives (pp. 368-383). Thousand Oaks, California: Sage.

Holzer, E., Tischler, L., Leaf, J., \& Myers, K. (1984). An epidemiologic assessment of cognitive impairment in a community. Research in Community Mental Health, 4, 3-32.

Hopp, A., Dixon, A., Grut, M., \& Backman, L. (1997). Longitudinal and psychometric profiles of two cognitive status tests in very old adults. Journal of Clinical Psychology, 53(7), 673-686.

Johnson, W., Logie, R. H., \& Brockmole, J. R. (2010). Working memory tasks differ in factor structure across age cohorts: implications for dedifferentiation. Intelligence, 38(5), 513-528.

Katsilambros, N., Steryotis, J., Moiras, N., Bezos, H., \& Daikos, G. (1977). Prevalence of Diabetes among glycosuric individuals in an urban area of Greece. Acta Diabetologica Latina, 14, 2211-2218.

Knol, J., Twisk, W., Beekman, T., Heine, J., Snoek, J., \& Pouwer, F. (2006). Depression as a risk factor for the onset of type 2 diabetes mellitus. A metaanalysis. Diabetologia, 49(5), 837-845.

Kryla-Lighthall, N. \& Mather, M. (2009). The role of cognitive control in older adults' emotional well-being. In V. Berngtson, D. Gans, N. Putney and M. Silverstein 
(Eds.), Handbook of Theories of Aging, (2nd Edition, pp. 323-344). New York: Springer.

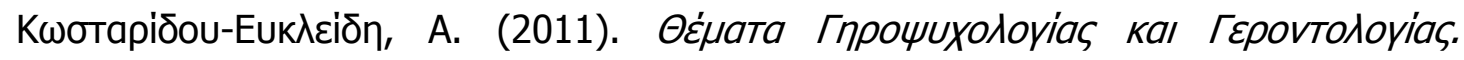

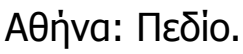

Lawton, P. (2001). Emotion in later life. Current Directions in Psychological Science, $10(4), 120-123$.

Liebert, R. \& Morris, L. (1967). Cognitive and emotional components of test anxiety: A distinction and some initial data. Psychological Reports, 20, 975-978.

Lowe, P. A., Lee, S. W., Witteborg, K. M., Prichard, K. W., Luhr, M. E., Cullinan, C. M., Mildren, B. A., Raad, J. M., Cornelius, R. A., \& Janik, M. (2008). The Test Anxiety Inventory for Children and Adolescents (TAICA): Examination of the psychometric properties of a new multidimensional measure of test anxiety among elementary and secondary school students. Journal of Psychoeducational Assessment, 26, 215-230.

Luciana, M., Collins, P., \& Depue, R. (1998). Opposing roles for dopamine and serotonin in the modulation of human spatial working memory functions. Cerebral Cortex, 8(3), 218-226.

Mandler, G. \& Sarason, S. B. ( 1952). A study of anxiety and learning. Journal of Abnormal and Social Psychology, 47, 166-173.

Metallidou, P. \& Vlachou, A. (2007). Motivational beliefs, cognitive engagement, and achievement in language and mathematics in elementary school children. International Journal of Psychology, 42, 2-15.

Meyer, A. \& Logan, J. (2013). Taking the testing effect beyond the college freshman: benefits for lifelong learning. Psychology and Aging, 28(1), 142-147.

Miles, D., Root, F., \& Hoston, D. (1922). Psychologic tests applied to diabetic patients. Archives of Internal Medicine, 30(6), 767-777.

Morales, L., Flowers, C., Gutierrez, P., Kleinman, M., \& Tenesi, J. (2006). Item and scale differential functioning of the Mini-Mental State Exam assessed using the differential item and test functioning (DFIT) framework. Medical Care, 44, 143151.

Mueller, J. H. (1992). Anxiety and performance. In A. P. Smith \& D. M. James (Eds.), Handbook of human performance. Vol 3: State and trait. London: Academic Press.

Owens, M., Stevenson, J., Hadwin, A., \& Norgate, R. (2012). Anxiety and depression in academic performance: An exploration of the mediating factors of worry and working memory. School Psychology International, 33(4), 433-449. 
Panagiotakos, D., Pitsavos, C., Chrysohoou, C., Risvas, G., Kontogianni, M., Zampelas, A., \& Stefanadis, C. (2005). The epidemiology of type 2 diabetes mellitus in a Greek adult: the ATTICA Study. Diabetic Medicine, 22, 1581-1588.

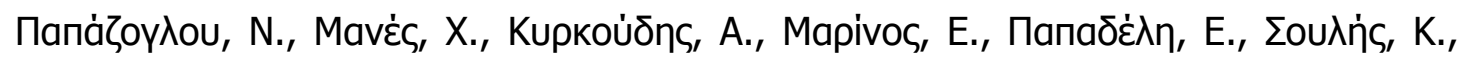

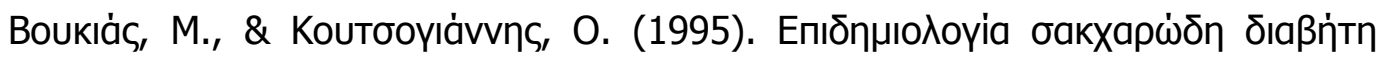

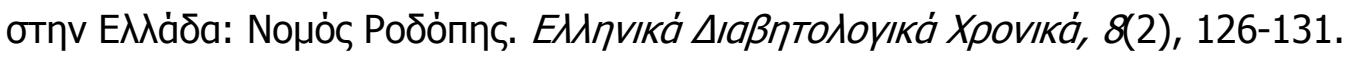

Papantoniou, G., Moraitou, D., \& Filippidou, D. (2011). Psychometric properties of Greek version of the Test Anxiety Inventory. Psychology, 2(3), 240-246.

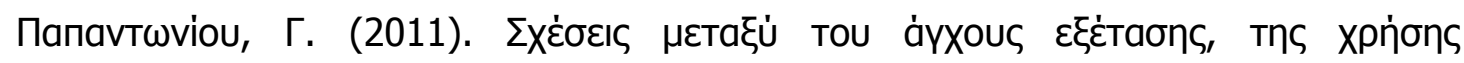

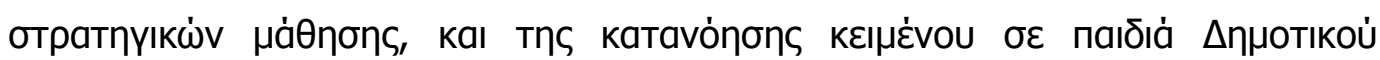

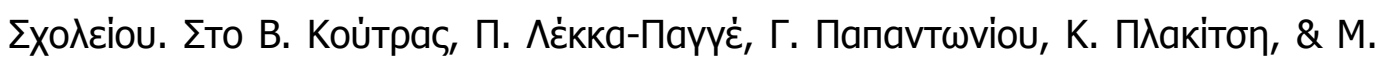

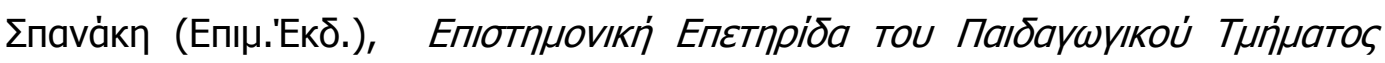

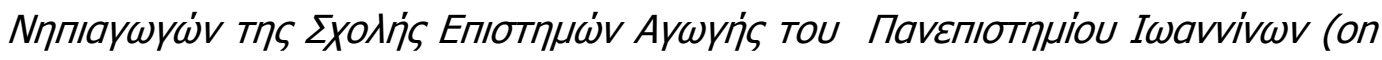
line). Tómos $\Delta$ ', 38-59.

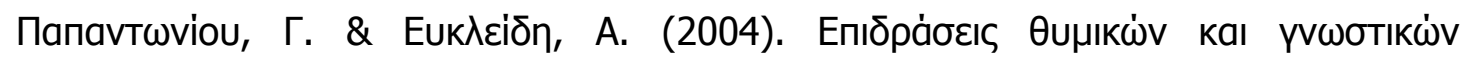

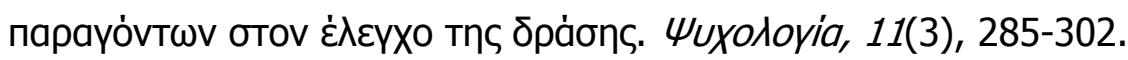

Peters, R. (2006). Ageing and the brain. Postgraduate Medical Journal, 82, 84-88.

Piccinin, A., Muniz-Terrera, G., Clouston, S., Reynolds, C., Thorvaldsson, V., Deary, I., Deeg, D., Johansson, B., Mackinnon, A., Spiro, A., Starr, J., Skoog, I., \& Hofer, S. (2013). Coordinated analysis of age, sex, and education effects on change in MMSE scores. The Journals of Gerontology: Series B, 68(3), 374-390.

Potvin, O., Bergua, V., Meillon, S., Le Goff, M., Buisson, J., Dartigues, J-F., \& Amieva, $\mathrm{H}$. (2013). State anxiety and cognitive functioning in older adults. The American Journal of Geriatric Psychiatry, 21(9), 915-924.

Salthouse, T. (1992). Shifting level of analysis in the investigation on cognitive aging. Human development, 35, 321-342.

Salthouse, T. (1996). Constraints on theories of cognitive aging. Psychonomic Bulletin \& Review, 3, 287-299.

Sarason, I. G. (1986). Test anxiety, worry and cognitive interference. In R. Schwarzer (Ed.), Self-related cognitions in anxiety and motivation (pp. 19-33). Hillsdale, NJ: Erlbaum.

Sarason, I. G., Pierce, G. R., \& Sarason, B. R. (1996). Domains of cognitive interference. In I. G. Sarason, G. R. Pierce, \& B. R. Sarason, B. R. (Eds.), Cognitive interference: Theories, methods, and findings (pp. 139-152). Mahwah, NJ: Erlbaum. 
Sarason, I. G., Sarason, B. R., Keefe, D. E., Hayes, B. E., \& Shearin, E. N. (1986). Cognitive interference: Situational determinants and trait-like characteristics. Journal of Personality and Social Psychology, 51(1), 215-226.

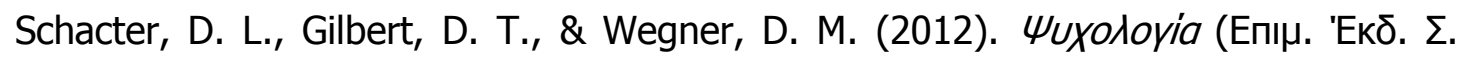
Booviádou). AӨn்va: Gutenberg.

Schaie, W. (2008). Historical processes and patterns of cognitive aging. In S. Hofer and D. Alwin (Eds.), Handbook of cognitive aging: interdisciplinary perspectives (pp. 368-383). Thousand Oaks, California: Sage.

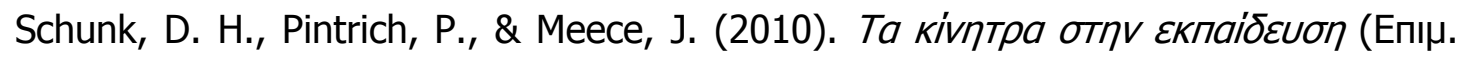

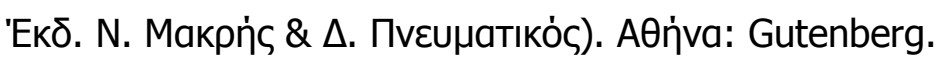

Schwarzer, R. (1984). Worry and emotionality as separate components in test anxiety. International Review of Applied Psychology, 33, 205-220.

Selye, H. (1956). The stress of life. New York: Mac-Graw Hill.

Selye, H. (1973). The evolution of stress concept: The originator of the concept traces its development from the discovery in 1936 of the alarm reaction to modern therapeutic applications of syntoxic and catatoxic hormones. American Scientist, 61(6), 692-699.

Shigemori, K., Ohgi, S., Okuyama, E., Shimura, T., \& Schneider, E. (2010). The factorial structure of the mini mental state examination (MMSE) in Japanese dementia patients. BMC Geriatrics, 10(36), 1471-2318.

Spielberger, C. (1966). Anxiety and behavior. New York: Academic Press.

Spielberger, C. (1972). Anxiety: Current trends in theory and research (Vol. II). New York: Academic Press.

Spielberger, C. (1979). Understanding stress and anxiety. New York: Harper \& Row.

Spielberger, C. D., Gonzalez, E. P., Taylor, C. J., Anton, W. D., Algaze, B., Ross, G. R., \& Westberry, L. G. (1979). Preliminary manual for the Test Anxiety Inventory. Palo Alto, CA: Consulting Psychologists Press.

Spiro, A. \& Brady, C. (2008). Integrating health into cognitive aging research and theory. In S. Hofer and D. Alwin (Eds.), Handbook of cognitive aging: interdisciplinary perspectives (pp. 260-283). Thousand Oaks, California: Sage.

Stillman, A., Rowe, K., Arndt, S., \& Moser, D. (2012). Anxious symptoms and cognitive function in non-demented older adults: an inverse relationship. International Journal of Geriatric Psychiatry, 27, 792-798. 


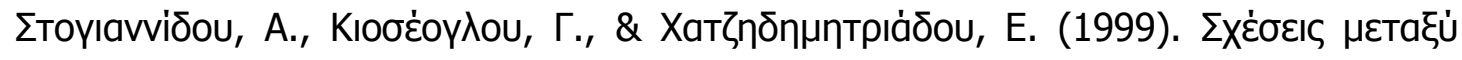

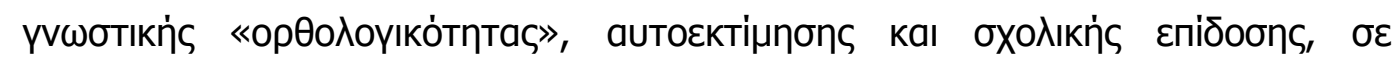

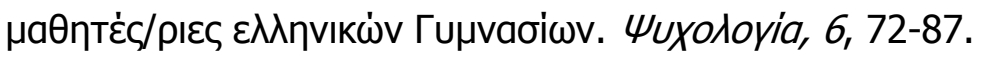

Sub, A. \& Prabha, C. (2003). Academic performance in relation to perfectionism, test procrastination and test anxiety of high school children. Psychological Studies, $48,7-81$.

Thomsen, D., Mehlsen, M., Viidik, A., Sommerlund, B., \& Zachariae, R. (2005). Age and gender differences in negative affect - Is there a role for emotion regulation? Personality and Individual Differences, 38, 1935-1946.

Tsolaki, M., Fountoulakis, K., Nakopoulou, E., Kazis, A., \& Mohs, R. C. (1997). Alzheimer's Disease Assessment Scale: the validation of the scale in Greece in elderly demented patients and normal subjects. Dementia and Geriatric Cognitive Disorders, $8(5)$, 273-80.

Uchino, B., Berg, S., Smith, T., Pearce, G., \& Skinner, M. (2006). Age-related differences in ambulatory blood pressure during daily stress: evidence for greater blood pressure reactivity with age. Psychology and Aging, 21(2), 231239.

Weinert, F. (1990). Theory building in the domain of motivation and learning in school. In P. Vedder (Ed.), Fundamental studies in educational research (pp. 91-120). Amsterdam: Swets \& Zeitlinger.

West, R. (1996). An application of prefrontal cortex function theory to cognitive aging. Psychological Bulletin, 120(2), 272-292.

Whitbourne, S. (1996). Aging; Aged; Psychological aspects; Psychology. New York: Springer.

Wigfield, A. \& Eccles, J. S. (1989). Test anxiety in elementary and secondary school students. Educational Psychologist, 24, 159-183

Willis, S. L. \& Blaskewicz Boron, J. (2008). Midlife cognition: the association of personality with cognition and risk of cognitive impairment. In S. Hofer and D. Alwin (Eds.), Handbook of cognitive aging: interdisciplinary perspectives (pp. 647-660). Thousand Oaks, California: Sage.

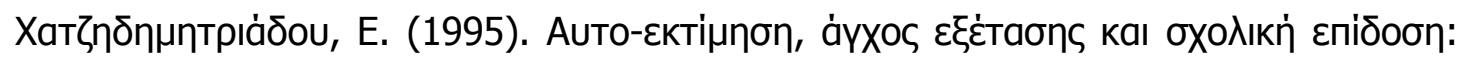

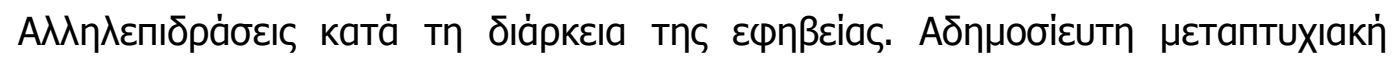

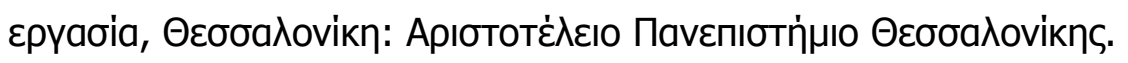


Yerkes, R. \& Dodson, J. (1908). The relation of strength of stimulus to rapidity of habit- formation. Journal of Comparative Neurology and Psychology, 18, 459482.

Zeidner, M. (1998). Test anxiety: The state of art. New York: Plenum. 